\title{
Lipschitz surfaces, perimeter and trace theorems for BV functions in Carnot-Carathéodory spaces
}

\author{
DAVIDE VitTONE
}

\begin{abstract}
We introduce intrinsic Lipschitz hypersurfaces in Carnot-Carathéodory spaces and prove that intrinsic Lipschitz domains have locally finite perimeter. We also show the existence of a boundary trace operator for functions with bounded variation on Lipschitz domains and obtain extension results for such functions. In particular, we characterize their trace space.
\end{abstract}

Mathematics Subject Classification (2010): 53C17 (primary); 46E35 (secondary).

\section{Introduction and statement of the main results}

In the last few years there has been an increasing interest towards analysis and geometry in metric spaces and, in particular, towards geometric measure theory and the study of spaces like those of Sobolev or bounded variation (BV) functions. In this paper we would like to give a contribution in these two directions, by dealing with the study of "Lipschitz regular" hypersurfaces and their relationship with the perimeter measure, and by establishing trace and extension theorems for BV functions in a metric setting. Our framework will be that of a Carnot-Carathéodory (CC) space, i.e., the space $\mathbb{R}^{n}$ endowed with the CC distance $d$ arising from a family $X=\left(X_{1}, \ldots, X_{m}\right)$ of smooth vector fields. See Section 2 for precise definitions.

In the setting of Carnot groups (see Section 3 for the definition), intrinsic Lipschitz surfaces have been introduced in [36,38] as graphs of intrinsic Lipschitz maps between complementary subgroups. For the case of codimension one, we propose here a new definition of Lipschitz surface which agrees with the previous one in Carnot groups (see Theorem 3.2) and can be stated in the more general framework of CC spaces.

The author is supported by MIUR, GNAMPA of INDAM (Italy) University of Padova, Fondazione CaRiPaRo Project "Nonlinear Partial Differential Equations: models, analysis, and control-theoretic problems" and University of Padova research project "Some analytic and differential geometric aspects in Nonlinear Control Theory, with applications to Mechanics".

Received October 18, 2010; accepted May 20, 2011. 
Definition 1.1. A set $S \subset \mathbb{R}^{n}$ is an $X$-Lipschitz surface if for any $x \in S$ there exist a neighbourhood $U$, a Lipschitz function $f: U \rightarrow \mathbb{R}$ and $j \in\{1, \ldots, m\}$ such that

$$
S \cap U=\{f=0\} \quad \text { and } \quad X_{j} f \geqslant l \quad \mathcal{L}^{n} \text {-a.e. on } U
$$

for a suitable $l>0$.

Notice that $X$-regular surfaces (see Section 2.4) are also $X$-Lipschitz.

To fix terminology, we will say that an open set $\Omega \subset \mathbb{R}^{n}$ is a $X$-Lipschitz domain if for any $x \in \partial \Omega$ there exist a neighbourhood $U$, a Lipschitz function $f: U \rightarrow \mathbb{R}$ and $j \in\{1, \ldots, m\}$ such that

- $\Omega \cap U=\{f>0\}$ or $\Omega \cap U=\{f<0\}$

- there exists $l>0$ such that $X_{j} f \geqslant l \mathcal{L}^{n}$-a.e. on $U$.

One of our main results is the following

Theorem 1.2. If $\Omega \subset \mathbb{R}^{n}$ is an $X$-Lipschitz domain, then $\Omega$ has locally finite $X$ perimeter in $\mathbb{R}^{n}$.

We refer to Section 2.3 for the definition of the $X$-perimeter measure $|\partial E|_{X}$ of a measurable subset $E \subset \mathbb{R}^{n}$. An easy consequence of Theorem 1.2 is the fact that subgraphs of one-codimensional intrinsic Lipschitz graphs in Carnot groups have locally finite $X$-perimeter, see Corollary 4.6. In the setting of the Heisenberg group, this fact has already been proved in [38] together with a Rademacher-type theorem for intrinsic Lipschitz graphs of codimension one. It would be very interesting to understand whether a Rademacher-type theorem holds for $X$-Lipschitz surfaces in a more general setting. A milder regularity result can be proved in equiregular $\mathrm{CC}$ spaces (see Section 4.2 for the definition), where $X$-Lipschitz surfaces are locally (and up to a diffeomorphism of the ambient space) graphs of Hölder continuous functions. See Proposition 4.10.

In equiregular $\mathrm{CC}$ spaces Theorem 1.2 can be refined to prove Ahlfors regularity of the $X$-perimeter of $X$-Lipschitz domains. Related results have been proved in [17-20, 24, 40] for more regular domains. We denote by $Q \in \mathbb{N}$ the Hausdorff dimension of $\left(\mathbb{R}^{n}, d\right)$.

Theorem 1.3. Let $\Omega$ be an $X$-Lipschitz domain with compact boundary in an equiregular CC space $\left(\mathbb{R}^{n}, X\right)$. Then the $X$-perimeter measure $|\partial \Omega|_{X}$ is $(Q-1)$ Ahlfors regular on $\partial \Omega$, i.e., there exist $\bar{r}>0$ and $\lambda>0$ such that

$$
\frac{1}{\lambda} r^{Q-1} \leqslant|\partial \Omega|_{X}(B(z, r)) \leqslant \lambda r^{Q-1} \text { for any } z \in \partial \Omega, 0<r<\bar{r} .
$$

As a consequence, we obtain that the $X$-perimeter measure of an $X$-Lipschitz domain with compact boundary is doubling, see Corollary 4.13. An asymptotic Ahlfors regularity of the perimeter measure, together with an asymptotic doubling property, was obtained by L. Ambrosio in [1]. The proofs of Theorems 1.2 and 1.3 , as well as many others in this papers, are based on the representation of the 
$X$-perimeter in certain local coordinates given by an implicit function theorem for $X$-Lipschitz surfaces, see Propositions 4.1 and 4.5. Here we have to acknowledge the influence of the analogous implicit function theorem proved in [22, Theorem 1.1] for $X$-regular surfaces.

The second part of this paper deals with trace theorems for functions of bounded $X$-variation in CC spaces. The theory of traces for Sobolev functions in this framework has been deeply investigated: here we mention [6-8, 11, 23-26, 49,58 ] and refer to the beautiful introduction of [26] for an account on the subject. On the contrary, the theory of traces for $B V_{X}$ functions in $\mathrm{CC}$ spaces is still at an early stage. To our best knowledge, trace and extension theorems for $B V_{X}$ functions have been established in [54] only for $H$-admissible domains (a class containing, for instance, $C^{1}$ domains with no characteristic points) in Carnot groups of step 2. We are able to prove trace and extension theorems for $B V_{X}$ functions defined on $X$-Lipschitz domains of a CC space.

Theorem 1.4. Let $\Omega \subset \mathbb{R}^{n}$ be an X-Lipschitz domain with compact boundary. Then, there exists a bounded linear operator

$$
T: B V_{X}(\Omega) \rightarrow L^{1}\left(\partial \Omega,|\partial \Omega|_{X}\right)
$$

such that

$$
\int_{\Omega} u \operatorname{div}_{X} g d \mathcal{L}^{n}=-\int_{\Omega}\left\langle\sigma_{u}, g\right\rangle d|X u|+\int_{\partial \Omega}\left\langle v_{\Omega}, g\right\rangle T u d|\partial \Omega|_{X}
$$

for any $u \in B V_{X}(\Omega)$ and $g \in C^{1}\left(\mathbb{R}^{n}, \mathbb{R}^{m}\right)$.

Here, $|X u|$ denotes the total $X$-variation of $u$ and $\sigma_{u}: \Omega \rightarrow S^{m-1}$ is the RadonNikodym derivative of the vector measure $X u$ with respect to $|X u|$, so that $X u=$ $\sigma_{u}|X u|$. Moreover, $\operatorname{div}_{X} g=X_{1}^{*} g_{1}+\cdots+X_{m}^{*} g_{m}$ and $\nu_{\Omega}$ is the generalized inward normal to $\Omega$. See Section 2.3 for precise definitions. The trace operator $T$ is not continuous if $B V_{X}(\Omega)$ is endowed with the topology of weak* convergence (see e.g. [2]). We prove in Theorem 5.6 that $T$ is instead continuous with respect to the so-called strict convergence.

Concerning the problem of the extension of $B V_{X}$ functions, we want to mention also the paper [9] were, in a more general framework, it is proved that the existence of an extension operator for $B V_{X}$ function on a domain $\Omega$ is equivalent to the validity of certain isoperimetric-type inequalities in $\Omega$. Here we prove the following

Theorem 1.5. Let $\Omega \subset \mathbb{R}^{n}$ be an X-Lipschitz domain with compact boundary. Then, there exists $C=C(\Omega)$ with the following property. For any $w \in L^{1}\left(\partial \Omega,|\partial \Omega|_{X}\right)$ and any $\delta>0$ there exists $u \in C^{\infty}(\Omega) \cap W_{X}^{1,1}(\Omega)$ such that

$$
T u=w, \quad \int_{\Omega}|u| d \mathcal{L}^{n} \leqslant \delta \quad \text { and } \quad \int_{\Omega}|X u| d \mathcal{L}^{n} \leqslant C\|w\|_{L^{1}\left(\partial \Omega,|\partial \Omega|_{X}\right)} .
$$


If $\partial \Omega$ is also $X$-regular, then $u$ can be chosen in such a way that

$$
\int_{\Omega}|X u| d \mathcal{L}^{n} \leqslant(1+\delta)\|w\|_{L^{1}\left(\partial \Omega,|\partial \Omega|_{X}\right)}
$$

We have denoted by $W_{X}^{1,1}(\Omega) \subset B V_{X}(\Omega)$ the space of functions $u \in L^{1}(\Omega)$ such that $X u \in L^{1}(\Omega)$. Let us point out that Theorems 1.4 and 1.5 characterize $L^{1}\left(\partial \Omega,|\partial \Omega|_{X}\right)$ as the trace space of $B V_{X}(\Omega)$ functions. Theorem 1.5 allows to obtain an extension result for $B V_{X}$ functions defined on $X$-Lipschitz domains, see Corollary 5.4.

Finally, we prove that, in equiregular CC spaces, the trace of $u$ on $\partial \Omega$ can be characterized in terms of the approximate limit of $u$ at points of $\partial \Omega$.

Theorem 1.6. Let $\left(\mathbb{R}^{n}, X\right)$ be an equiregular $C C$ space, $\Omega \subset \mathbb{R}^{n}$ an X-Lipschitz domain with compact boundary and $u \in B V_{X}(\Omega)$. Then

$$
\lim _{r \rightarrow 0^{+}} \frac{1}{r Q} \int_{\Omega \cap B(z, r)}|u-T u(z)| d \mathcal{L}^{n}=0 \quad \text { for }|\partial \Omega|_{X} \text {-a.e. } z \in \partial \Omega
$$

and in particular

$$
T u(z)=\lim _{r \rightarrow 0^{+}} f_{\Omega \cap B(z, r)} u d \mathcal{L}^{n} \quad \text { for }|\partial \Omega|_{X} \text {-a.e. } z \in \partial \Omega .
$$

The paper is organized as follows. In Section 2 we introduce the basic notions on CC spaces, functions with bounded $X$-variation and sets with finite $X$-perimeter. The equivalence between $X$-Lipschitz surfaces and intrinsic Lipschitz graphs in Carnot groups is the object of Section 3. Section 4.1 is devoted to the study of $X$-Lipschitz surfaces and the proof of Theorem 1.2, while Theorem 1.3 is proved in Section 4.2. Finally, Theorems 1.4, 1.5 and 1.6 are proved in Section 5 together with the aforementioned related result.

ACKNOWLEDGEMENTS. It is a great pleasure to thank E. Spadaro for many illuminating discussions. The author is also grateful to R. Monti, R. Serapioni and F. Serra Cassano for their interest in the paper and for several stimulating discussions.

\section{Notation and preliminary results}

We briefly introduce Carnot-Carathéodory spaces and refer to [10] for a more general account on the subject. 


\subsection{Carnot-Carathéodory spaces}

Let $X=\left(X_{1}, \ldots, X_{m}\right)$ be a fixed family of $C^{\infty}$ vector fields in $\mathbb{R}^{n}$. As common in the literature, we will systematically identify vector fields and first order differential operators. We call horizontal (at a given point $x \in \mathbb{R}^{n}$ ) any vector that is a linear combination of $X_{1}(x), \ldots, X_{m}(x)$. An absolutely continuous curve $\gamma:[0, T] \longrightarrow$ $\mathbb{R}^{n}$ is sub-unit if

$$
\dot{\gamma}(t)=\sum_{j=1}^{m} h_{j}(t) X_{j}(\gamma(t)) \quad \text { and } \quad \sum_{j=1}^{m} h_{j}^{2}(t) \leqslant 1 \quad \text { for a.e. } t \in[0, T],
$$

with $h_{1}, \ldots, h_{m}$ measurable coefficients.

Definition 2.1. We define the Carnot-Carathéodory distance $d$ between $x, y \in \mathbb{R}^{n}$ as

$$
d(x, y)=\inf \left\{T \geqslant 0: \begin{array}{l}
\text { there exists a sub-unit path } \gamma:[0, T] \rightarrow \mathbb{R}^{n} \\
\text { such that } \gamma(0)=x \text { and } \gamma(T)=y
\end{array}\right\} .
$$

If the above set is empty we set $d(x, y)=+\infty$.

If $d(x, y)<\infty$ for every $x, y \in \mathbb{R}^{n}$, then $d$ is a distance on $\mathbb{R}^{n}$. We shall generally assume that

$d$ is finite and the identity map $\left(\mathbb{R}^{n}, d\right) \rightarrow\left(\mathbb{R}^{n},|\cdot|\right)$ is a homeomorphism.

Condition (2.1) holds, for example, when the Chow-Hörmander condition

$$
\operatorname{rank} \mathfrak{L}\left(X_{1}, \ldots, X_{m}\right)(x)=n
$$

is satisfied for any $x \in \mathbb{R}^{n}$ (see [52]); here, $\mathfrak{L}\left(X_{1}, \ldots, X_{m}\right)$ denotes the Lie algebra generated by $X_{1}, \ldots, X_{m}$ and their commutators of any order. We will use the notation $B(x, r)$ for balls with respect to the $\mathrm{CC}$ distance, while Euclidean balls in $\mathbb{R}^{k}$ are denoted by $\mathscr{B}(x, r)$.

Given $E \subset \mathbb{R}^{n}$ and $k \geqslant 0$, the $k$-dimensional Hausdorff and spherical Hausdorff measures of $E$ are defined, respectively, by

$$
\begin{aligned}
& \mathcal{H}_{d}^{k}(E):=\liminf _{\delta \downarrow 0}\left\{\sum_{i=0}^{\infty}\left(\operatorname{diam} E_{i}\right)^{k}: E \subset \cup_{i=0}^{\infty} E_{i}, \operatorname{diam} E_{i}<\delta\right\} \\
& \mathcal{S}_{d}^{k}(E):=\lim _{\delta \downarrow 0} \inf \left\{\sum_{i=0}^{\infty}\left(\operatorname{diam} B_{i}\right)^{k}: E \subset \cup_{i=0}^{\infty} B_{i}, \operatorname{diam} B_{i}<\delta, B_{i} \subset \mathbb{R}^{n} \text { balls }\right\} .
\end{aligned}
$$

The standard Euclidean Hausdorff measures in $\mathbb{R}^{n}$ will instead be denoted by $\mathcal{H}^{k}, \mathcal{S}^{k}$. 


\subsection{Lipschitz and $C_{X}^{1}$ functions}

When $u: \Omega \rightarrow \mathbb{R}$ is a measurable function on an open set $\Omega \subset \mathbb{R}^{n}$ we define its horizontal gradient $X u$ as

$$
X u:=\left(X_{1} u, \ldots, X_{m} u\right),
$$

where the derivatives are to be understood in the sense of distributions. It is well known that, if $u: \Omega \rightarrow \mathbb{R}$ is Lipschitz continuous with respect to $d$, then $X u \in$ $L^{\infty}(\Omega)$. Viceversa (see $[32,40]$ ), if $u$ is continuous and $X u \in L^{\infty}(\Omega)$, then $u$ is Lipschitz on any open set $\Omega^{\prime} \Subset \Omega$.

We will say that $u$ is of class $C_{X}^{1}(\Omega)$ if $u$ and $X u$ are continuous. If $u$ is of class $C_{X}^{1}$, then it is differentiable (in the classical sense) along the vector fields $X_{1}, \ldots, X_{m}$.

In the sequel, we will use several times the following simple lemma, whose proof is given for the sake of completeness.

Lemma 2.2. Let $f: \mathbb{R}^{n} \rightarrow \mathbb{R}$ be a continuous function and $Y$ a vector field in $\mathbb{R}^{n}$ with smooth coefficients. Assume that $Y f \geqslant l$ holds, in the sense of distributions, on an open set $U \subset \mathbb{R}^{n}$ and for a suitable positive constant l. If $x \in \mathbb{R}^{n}$ and $h_{1}<h_{2}$ are such that $\exp (h Y)(x) \in U$ for any $h \in\left(h_{1}, h_{2}\right)$, then

$$
f(\exp (t Y)(x)) \geqslant f(\exp (s Y)(x))+l(t-s) \quad \text { for any } t, s \in\left(h_{1}, h_{2}\right) \text { with } s<t .
$$

In particular, if there exists $t \in\left(h_{1}, h_{2}\right)$ such that $f(\exp (t Y)(x))=0$, then such a $t$ is unique.

Proof. Up to a smooth change of coordinates (see also the proof of Proposition 4.1, where a similar argument is used) we may assume that there exists a neighbourhood $V \subset U$ of the compact set $\{\exp (h Y)(x): h \in[s, t]\}$ such that $Y=e_{n}=$ $(0, \ldots, 0,1)$ on $V$. Therefore, for any $h \in[s, t]$ we have $x_{h}:=\exp (h Y)(x)=$ $x_{s}+(h-s) e_{n}$.

For $k \in \mathbb{N}$ let $\psi_{k} \in C_{c}^{\infty}\left(\mathscr{B}\left(x_{s}, \frac{1}{k}\right)\right)$ be such that $\psi_{k} \geqslant 0$ and $\int \psi_{k} d \mathcal{L}^{n}=1$. For any $h \in[s, t]$ define $\tau_{h} \psi_{k}(y):=\psi_{k}\left(y-(h-s) e_{n}\right)$. If $k$ is large enough, then $\tau_{h} \psi_{k}$ has support in $V$ for any $h \in[s, t]$. Clearly, as $k \rightarrow \infty$ the functions $\tau_{h} \psi_{k}$ converge to the Dirac delta at $x_{h}$.

Since the inequality $\partial_{x_{n}} f \geqslant l$ holds in the sense of distributions on $V$, by the continuity of $f$ we have

$$
\begin{aligned}
f\left(x_{t}\right)-f\left(x_{s}\right) & =\lim _{k \rightarrow \infty}\left(\int_{V} f \tau_{t} \psi_{k} d \mathcal{L}^{n}-\int_{V} f \tau_{s} \psi_{k} d \mathcal{L}^{n}\right) \\
& =\lim _{k \rightarrow \infty} \int_{s}^{t} \frac{d}{d h}\left(\int_{V} f \tau_{h} \psi_{k} d \mathcal{L}^{n}\right) d h \\
& =\lim _{k \rightarrow \infty} \int_{s}^{t}\left(-\int_{V} f(y) \frac{\partial \psi_{k}}{\partial x_{n}}\left(y-h e_{n}\right) d y\right) d h \\
& \geqslant \lim _{k \rightarrow \infty} \int_{s}^{t} l \int_{V} \psi_{k}\left(y-t e_{n}\right) d y d h=l(s-t)
\end{aligned}
$$

and the lemma follows. 


\subsection{Functions with bounded $X$-variation and $X$-perimeter}

The space of functions with bounded $X$-variation has been considered in several papers, see e.g. $[14,16,24,31,39]$. If $g=\left(g_{1}, \ldots, g_{m}\right) \in C_{c}^{1}\left(\Omega ; \mathbb{R}^{m}\right)$ we set $\operatorname{div}_{X} g:=\sum_{j=1}^{m} X_{j}^{*} g_{j}$, where $X_{j}^{*}$ is the formal adjoint operator of $X_{j}$ given by

$$
X_{j}^{*} \psi(x)=\sum_{i=1}^{n} \frac{\partial\left(a_{i j} \psi\right)}{\partial x_{i}}(x) \quad \forall \psi \in C^{1}\left(\mathbb{R}^{n}\right)
$$

and where we have set $X_{j}(x)=\left(a_{1 j}(x), \ldots, a_{n j}(x)\right)$. Notice that the $m$-vector function $g$ can be canonically identified with a section of the horizontal bundle, namely $g_{1} X_{1}+\cdots+g_{m} X_{m}$.

Definition 2.3. Let $\Omega$ be an open subset of $\mathbb{R}^{n}$; we say that $u \in L^{1}(\Omega)$ has bounded $X$-variation in $\Omega$ if

$$
|X u|(\Omega):=\sup \left\{\int_{\Omega} u \operatorname{div}_{X} g d \mathcal{L}^{n}: g \in C_{c}^{1}\left(\Omega, \mathbb{R}^{m}\right),|g| \leqslant 1\right\}
$$

is finite. The space of functions with bounded $X$-variation in $\Omega$ is denoted by $B V_{X}(\Omega)$.

It is well known that $u$ belongs to $B V_{X}(\Omega)$ if and only if $X u$ is represented by a Radon vector measure $\mu=\left(\mu_{1}, \ldots, \mu_{m}\right)$ on $\Omega$ with finite total variation. Moreover, the measure $|X u|$ coincides with the total variation ${ }^{1}|\mu|$ of $\mu$ and there exists a $|X u|$-measurable function $\sigma_{u}: \Omega \rightarrow S^{m-1}$ such that $\mu=X u=\sigma_{u}|X u|$ and

$$
\int_{\Omega} u \operatorname{div}_{X} g d \mathcal{L}^{n}=-\sum_{j=1}^{m} \int_{\Omega} g_{j} d \mu_{j}=-\int_{\Omega}\left\langle g, \sigma_{u}\right\rangle d|X u|
$$

for all $g \in C_{c}^{1}\left(\Omega, \mathbb{R}^{m}\right)$. The space $B V_{X}(\Omega)$ is a Banach space when endowed with the norm

$$
\|u\|_{B V_{X}(\Omega)}:=\|u\|_{L^{1}(\Omega)}+|X u|(\Omega) .
$$

We also introduce the Sobolev space $W_{X}^{1,1}(\Omega)$ as the space of those functions $u \in$ $L^{1}(\Omega)$ such that $X u$ is represented by a function in $L^{1}\left(\Omega, \mathbb{R}^{m}\right)$. It is a Banach space if endowed with the norm

$$
\|u\|_{W_{X}^{1,1}(\Omega)}:=\|u\|_{L^{1}(\Omega)}+\|X u\|_{L^{1}(\Omega)}
$$

and, clearly, $W_{X}^{1,1}(\Omega) \subset B V_{X}(\Omega)$.

${ }^{1}$ Recall that the total variation of $\mu$ is defined by

$$
|\mu|(A):=\sup \left\{\sum_{i=1}^{\infty}\left|\mu\left(A_{i}\right)\right|: A=\cup_{i} A_{i}, A i \text { disjoint }\right\} .
$$


It follows from (2.2) that the total $X$-variation on open sets is lower semicontinuous with respect to the $L^{1}$-convergence, i.e., if $u, u_{k} \in L^{1}(\Omega)$ are such that $u_{k} \rightarrow u$ in $L^{1}(\Omega)$, then

$$
|X u|(\Omega) \leqslant \liminf _{k \rightarrow \infty}\left|X u_{k}\right|(\Omega) .
$$

We will say that a sequence $\left(u_{k}\right)_{k} \subset B V_{X}(\Omega)$ strictly converges to $u \in B V_{X}(\Omega)$ if

$$
u_{k} \rightarrow u \text { in } L^{1}(\Omega) \quad \text { and } \quad\left|X u_{k}\right|(\Omega) \rightarrow|X u|(\Omega) .
$$

It was proved in $[31,39]$ that $u \in L^{1}(\Omega)$ has bounded $X$-variation in $\Omega$ if and only if there exists a sequence $\left(u_{k}\right)_{k} \subset C^{\infty}(\Omega) \cap B V_{X}(\Omega)$ such that $u_{k} \rightarrow u$ strictly.

Strict convergence guarantees upper semicontinuity of the total $X$-variation on closed sets; actually, under some additional assumption it provides also the continuity of the total $X$-variation on open sets, as stated in the following lemma.

Lemma 2.4. Let $\Omega \subset \mathbb{R}^{n}$ be open and $u, u_{k} \in B V_{X}(\Omega)(k \in \mathbb{N})$ such that

$$
u_{k} \rightarrow u \text { in } L^{1}(\Omega) \text { and }\left|X u_{k}\right|(\Omega) \rightarrow|X u|(\Omega)
$$

as $k \rightarrow \infty$. Then, for any relatively closed set $C \subset \Omega$ (i.e., if $\Omega \backslash C$ is open) we have

$$
|X u|(C) \geqslant \limsup _{k \rightarrow \infty}\left|X u_{k}\right|(C) .
$$

Moreover, if $U \subset \Omega$ is an open set such that $|X u|(\partial U)=0$ we have

$$
|X u|(U)=\lim _{k \rightarrow \infty}\left|X u_{k}\right|(U) .
$$

Proof. We have

$$
\begin{aligned}
\limsup _{k \rightarrow \infty}\left|X u_{k}\right|(C) & =\limsup _{k \rightarrow \infty}\left[\left|X u_{k}\right|(\Omega)-\left|X u_{k}\right|(\Omega \backslash C)\right] \\
& =|X u|(\Omega)-\liminf _{k \rightarrow \infty}\left|X u_{k}\right|(\Omega \backslash C) \\
& \leqslant|X u|(\Omega)-|X u|(\Omega \backslash C)=|X u|(C)
\end{aligned}
$$

and the first part of the statement is proved. Thus, if $|X u|(\partial U)=0$ we get also

$$
|X u|(U)=|X u|(\bar{U} \cap \Omega) \geqslant \limsup _{k \rightarrow \infty}\left|X u_{k}\right|(\bar{U} \cap \Omega) \geqslant \liminf _{k \rightarrow \infty}\left|X u_{k}\right|(U) \geqslant|X u|(U)
$$

and the proof is accomplished.

It is convenient to introduce also the notion of weak* convergence in $B V_{X}$. A sequence $\left(u_{k}\right)_{k}$ weakly* converges to $u \in B V_{X}(\Omega)$ if $u_{k} \rightarrow u$ in $L^{1}(\Omega)$ and $\left(X u_{k}\right)_{k}$ weakly* converges to $X u$ in $\Omega$, i.e.,

$$
\lim _{k \rightarrow \infty} \int_{\Omega} \eta d X u_{k}=\int_{\Omega} \eta d X u \quad \text { for any } \eta \in C_{0}(\Omega)
$$

where $C_{0}(\Omega)$ is the closure of $C_{c}^{0}(\Omega)$ in the sup norm. 
As the following result shows, strict convergence implies weak* convergence. We use the standard notation $C_{b}(\Omega)$ to denote the vector space of continuous and bounded real functions on $\Omega$.

Lemma 2.5. Assume that $u, u_{k} \in B V_{X}(\Omega)$ are such that $u_{k} \rightarrow u$ in $L^{1}(\Omega)$ and $\left|X u_{k}\right|(\Omega) \rightarrow|X u|(\Omega)$. Then

$$
\lim _{k \rightarrow \infty} \int_{\Omega} \eta d X_{\ell} u_{k}=\int_{\Omega} \eta d X_{\ell} u \quad \text { for any } \eta \in C_{b}(\Omega) \text { and } \ell=1, \ldots, m .
$$

Proof. We follow the proof of [2, Proposition 3.15] and prove, more generally, that for any continuous and positively 1 -homogeneous function $F: \mathbb{R}^{m} \rightarrow \mathbb{R}$ it holds

$$
\lim _{k \rightarrow \infty} \int_{\Omega} \eta F\left(\sigma_{u_{k}}\right) d\left|X_{\ell} u_{k}\right|=\int_{\Omega} \eta F\left(\sigma_{u}\right) d\left|X_{\ell} u\right| \quad \forall \eta \in C_{b}(\Omega), \ell=1, \ldots, m .
$$

Equality (2.3) follows on choosing $F\left(\sigma_{1}, \ldots, \sigma_{m}\right):=\sigma_{\ell}$.

Possibly splitting $F$ in positive and negative part we can assume with no loss of generality that $F \geqslant 0$. By [2, Proposition 1.80] we obtain that

$$
\lim _{k \rightarrow \infty} \int_{\Omega} \eta d\left|X u_{k}\right|=\int_{\Omega} \eta d|X u| \text { for any } \eta \in C_{0}(\Omega) .
$$

In particular, we can apply Reshetnyak continuity theorem (see [2, Theorem 2.39]) to get

$$
\lim _{k \rightarrow \infty} \int_{\Omega} F\left(\sigma_{u_{k}}\right) d\left|X u_{k}\right|=\int_{\Omega} F\left(\sigma_{u}\right) d|X u|
$$

More generally: for any $\Omega^{\prime} \subset \Omega$ such that $|X u|\left(\partial \Omega^{\prime}\right)=0$, we have by Lemma 2.4 that $\left|X u_{k}\right|\left(\Omega^{\prime}\right) \rightarrow|X u|\left(\Omega^{\prime}\right)$ and, reasoning as before, we obtain

$$
\lim _{k \rightarrow \infty} \int_{\Omega^{\prime}} F\left(\sigma_{u_{k}}\right) d\left|X u_{k}\right|=\int_{\Omega^{\prime}} F\left(\sigma_{u}\right) d|X u| .
$$

Taking into account that any open set $\Omega^{\prime} \subset \Omega$ can be approximated from inside by a sequence $\left(\Omega_{h}^{\prime}\right)_{h}$ of open sets with $|X u|\left(\partial \Omega_{h}^{\prime}\right)=0$, we get

$$
\liminf _{k \rightarrow \infty} \int_{\Omega^{\prime}} F\left(\sigma_{u_{k}}\right) d\left|X u_{k}\right| \geqslant \int_{\Omega^{\prime}} F\left(\sigma_{u}\right) d|X u|
$$

and (2.4) follows from [2, Proposition 1.80].

Following the classical approach to sets of finite perimeter à la Caccioppoli-De Giorgi, as in $[16,30,31,39]$ we define the $X$-perimeter measure $|\partial E|_{X}$ of a measurable set $E \subset \mathbb{R}^{n}$ as the $X$-variation of its characteristic function $\chi_{E}$. Namely, for any open set $\Omega \subset \mathbb{R}^{n}$ we define

$$
|\partial E|_{X}(\Omega):=\sup \left\{\int_{E} \operatorname{div}_{X} g d \mathcal{L}^{n}: g \in C_{c}^{1}\left(\Omega, \mathbb{R}^{m}\right),|g| \leqslant 1\right\} .
$$


Clearly, $E$ has finite $X$-perimeter in $\Omega$ if and only if $\chi_{E} \in B V_{X}(\Omega)$. Open sets with smooth boundary have locally finite $X$-perimeter and representation formulae for the associated measure are available, see e.g. $[16,31,44]$. Notice that the measure $|\partial E|_{X}$ is invariant under modifications of $E$ on $\mathcal{L}^{n}$-negligible sets and that $|\partial E|_{X}=$ $\left|\partial\left(\mathbb{R}^{n} \backslash E\right)\right|_{X}$

If $E$ has finite perimeter in $\Omega$, then the $|\partial E|_{X}$-measurable function $\nu_{E}:=\sigma_{\chi_{E}}$ : $\Omega \rightarrow S^{m-1}$ satisfies

$$
\int_{E} \operatorname{div}_{X} g d \mathcal{L}^{n}=-\int_{\Omega}\left\langle g, v_{E}\right\rangle d|\partial E|_{X} \quad \text { for any } g \in C_{c}^{1}\left(\Omega, \mathbb{R}^{m}\right) .
$$

The map $v_{E}$ is called horizontal inward normal to $E$.

The following coarea formula, which will be used extensively throughout the paper, was proved in [50].

Theorem 2.6. Suppose that the vector fields $X_{1}, \ldots, X_{m}$ satisfy assumption (2.1). Let $f: \mathbb{R}^{n} \rightarrow \mathbb{R}$ be Lipschitz continuous with respect to $d$ and let $u: \mathbb{R}^{n} \rightarrow$ $[0,+\infty]$ be $\mathcal{L}^{n}$-measurable. Then

$$
\int_{\mathbb{R}^{n}} u(x)|X f(x)| d x=\int_{-\infty}^{+\infty} \int_{\{f=s\}} u d\left|\partial E_{s}\right|_{X} d s,
$$

where $E_{s}:=\{f<s\}$.

\subsection{Regular surfaces in $\mathrm{CC}$ spaces}

Intrinsic regular surfaces have been introduced in [22,33, 35], in different settings, as noncritical level set of $C_{X}^{1}$ functions. We say that $S \subset \mathbb{R}^{n}$ is a $X$-regular surface if for any $x \in S$ there exist a neighbourhood $U$ and $f \in C_{X}^{1}(U)$ such that

$$
S \cap U=\{f=0\} \quad \text { and } \quad X f \neq 0 \text { on } U .
$$

A Euclidean smooth hypersurface $\Sigma$ is $X$-regular provided it contains no characteristic points, i.e. points $x \in \Sigma$ such that

$$
\operatorname{span}\left(X_{1}(x), \ldots, X_{m}(x)\right) \subset \operatorname{Tan}_{x} \Sigma
$$

where $\operatorname{Tan}_{x} \Sigma$ denotes the Euclidean tangent hyperplane to $\Sigma$ at $x$. On the contrary, genuine $X$-regular surfaces can be very far from being Euclidean regular, as they may have a fractal behaviour (see [42]). The importance of $X$-regular surfaces arises evident in the theory of rectifiability (see [33]). The problem of the intrinsic measure of surfaces in $\mathrm{CC}$ spaces has been attacked in several papers like $[3,16$, $22,44,45,50]$, but this list is surely incomplete. Clearly, $X$-regular surfaces are $X$-Lipschitz according to Definition 1.1.

We will say that an open set $\Omega \subset \mathbb{R}^{n}$ is a $X$-regular domain if for any $x \in \partial \Omega$ there exist a neighbourhood $U$ and a function $f \in C_{X}^{1}(U)$ such that

- $\Omega \cap U=\{f>0\}$ or $\Omega \cap U=\{f<0\}$

- $X f \neq 0$ on $U$. 


\section{3. $X$-Lipschitz surfaces and intrinsic Lipschitz graphs in Carnot groups}

A Carnot group $\mathbb{G}$ of step $\kappa$ (see e.g. $[15,28,29,43,53,56,57,59])$ is a $n$-dimensional connected and simply connected Lie group whose Lie algebra $\mathfrak{g}$ admits a step $\kappa$ stratification, i.e., there exist linear subspaces $V_{1}, \ldots, V_{\kappa} \subset \mathfrak{g}$ such that

$$
\mathfrak{g}=V_{1} \oplus \cdots \oplus V_{\kappa}, \quad\left[V_{1}, V_{i}\right]=V_{i+1}, \quad V_{\kappa} \neq\{0\}, \quad\left[V_{1}, V_{\kappa}\right]=\{0\},
$$

where $\left[V_{1}, V_{i}\right]$ is the subspace of $\mathfrak{g}$ generated by the commutators $[X, Y]$ with $X \in$ $V_{1}$ and $Y \in V_{i}$.

Let $m_{i}:=\operatorname{dim}\left(V_{i}\right), n_{i}:=m_{1}+\cdots+m_{i}(i=1, \ldots, \kappa)$ and $n_{0}=0$; clearly, $n_{\kappa}=n$. Choose a basis $v_{1}, \ldots, v_{n}$ of $\mathfrak{g}$ adapted to the stratification, that is, such that

$$
v_{n_{i-1}+1}, \ldots, v_{n_{i}} \text { is a basis of } V_{i} \text { for any } i=1, \ldots, \kappa \text {. }
$$

Let $\left(X_{1}, \ldots, X_{n}\right)$ be the family of left invariant vector fields such that $X_{i}(0)=$ $v_{i}$. By (3.1), the family $X=\left(X_{1}, \ldots, X_{m}\right)\left(m:=m_{1}\right)$ Lie generates the whole algebra $\mathfrak{g}$ and the Chow-Hörmander condition is satisfied. We endow $\mathbb{G}$ with the CC structure induced by $X$; in this way, $\mathbb{G}$ is an equiregular $C C$ space (see Section 4.2). The homogeneous dimension of $\mathbb{G}$ is $Q=\sum_{i=1}^{k} i m_{i}$ and this integer coincides with the Hausdorff dimension of the CC space $\mathbb{G}$ (see [48]).

The exponential map is a diffeomorphism from $\mathfrak{g}$ onto $\mathbb{G}$, i.e. any $x \in \mathbb{G}$ can be written in a unique way as $x=\exp \left(x_{1} X_{1}+\cdots+x_{n} X_{n}\right)$. Using these exponential coordinates, we identify $x \in \mathbb{G}$ with the $n$-tuple $\left(x_{1}, \ldots, x_{n}\right) \in \mathbb{R}^{n}$ and, accordingly, $\mathbb{G}$ with $\mathbb{R}^{n}$. In this way, the group identity is the origin of $\mathbb{R}^{n}$ and the Haar measure of $\mathbb{G}$ is the Lebesgue measure $\mathcal{L}^{n}$. The explicit expression of the group operation, which we denote by $\cdot$, is determined by the Baker-CampbellHausdorff formula and, in exponential coordinates, takes the form

$$
x \cdot y=x+y+Q(x, y)
$$

for suitable polynomial functions $Q_{1}, \ldots, Q_{n}$. It is well known that $Q_{i}(x, y)=$ 0 for any $i=1, \ldots, m$, i.e., the group operation is commutative in the first $m$ coordinates.

Recall that $\mathbb{G}$ is endowed with a one-parameter family $\left(\delta_{r}\right)_{r>0}$ of dilations which, in exponential coordinates, read as

$$
\delta_{r}\left(x_{1}, \ldots, x_{n}\right)=\left(r x_{1}, \ldots, r^{d(i)} x_{i}, \ldots, r^{\kappa} x_{n}\right),
$$

where, for $i=1, \ldots, n, d(i)$ is defined by $X_{i} \in V_{d(i)}$. A function $f: \mathbb{G} \rightarrow \mathbb{R}$ is homogeneous of degree $d$ (briefly: $d$-homogeneous) if $f \circ \delta_{r}=r^{d} f$ for any $r>0$. If $f$ is $d$-homogeneous and $C^{1}$ regular, then $X f$ is $(d-1)$-homogeneous.

Let us introduce the pseudo-norm

$$
\|x\|_{\mathbb{G}}:=\left(\sum_{i=1}^{n}\left|x_{i}\right|^{Q / d(i)}\right)^{1 / Q}, \quad x=\left(x_{1}, \ldots, x_{n}\right) \in \mathbb{G} .
$$


The map $x \mapsto\|x\|_{\mathbb{G}}$ is 1 -homogeneous, continuous on $\mathbb{G}$ and of class $C^{1}$ on the open set $\mathbb{G} \backslash\{0\}$ because $Q / d(i)>1$. Consequently, there exists $c>0$ such that

$$
\frac{1}{c}\left\|x^{-1} \cdot y\right\|_{\mathbb{G}} \leqslant d(x, y) \leqslant c\left\|x^{-1} \cdot y\right\|_{\mathbb{G}} \quad \text { for any } x, y \in \mathbb{G} .
$$

The set $\mathbb{W}:=\exp \left(\operatorname{span}\left\{X_{2}, \ldots, X_{n}\right\}\right)$ is a normal, 1-codimensional maximal subgroup of $\mathbb{G}$. For any $x \in \mathbb{G}$, there exists a unique $x_{\mathbb{W}} \in \mathbb{W}$ such that $x=$ $x_{\mathbb{W}} \cdot x_{1} e_{1}$, where for $h \in \mathbb{R}$ we set $h e_{1}:=(h, 0, \ldots, 0) \in \mathbb{G}$. Clearly, one has $x_{\mathbb{W}}=x \cdot\left(-x_{1} e_{1}\right)=\exp \left(-x_{1} X_{1}\right)(x)$. We also point out that

$$
x \cdot s e_{1}=\exp \left(s X_{1}\right)(x) \quad \text { for any } x \in \mathbb{G}, s \in \mathbb{R} .
$$

For $\alpha>0$, the homogeneous open cone $\mathcal{C}_{\alpha}$ along $X_{1}$ of center 0 and aperture $1 / \alpha$ is defined as

$$
\mathcal{C}_{\alpha}:=\left\{x \in \mathbb{G}:\left|x_{1}\right|>\alpha\left\|x_{\mathbb{W}}\right\|_{\mathbb{G}}\right\} ;
$$

we also introduce

$$
\mathcal{C}_{\alpha}^{+}:=\left\{x \in \mathcal{C}_{\alpha}: x_{1}>0\right\} .
$$

Let $\omega \subset \mathbb{W}$ and $\phi: \omega \rightarrow \mathbb{R}$; the intrinsic graph (along $X_{1}$ ) of $\phi$ is the image $\Phi(\omega) \subset \mathbb{G}$ of the map

$$
\Phi(y):=y \cdot \phi(y) e_{1}, \quad y \in \omega .
$$

In a similar way it is possible to define intrinsic graphs along any vector field $X_{j}$, $j \in\{1, \ldots, m\}$. It turns out that, if $S \subset \mathbb{G}$ is an intrinsic graph along $X_{j}$, then for any $x \in \mathbb{G}$ the left translation $x \cdot S$ is an intrinsic graph along $X_{j}$. Without loss of generality, however, here and in the following we will treat only intrinsic graphs along $X_{1}$. For more details, see [37].

According to $[5,36,38]$, we say that $\phi$ is intrinsic Lipschitz (and that $\Phi(\omega)$ is an intrinsic Lipschitz graph) if there exists $\alpha>0$ such that

$$
\Phi(\omega) \cap x \cdot \overline{\mathcal{C}_{\alpha}}=\{x\} \quad \text { for any } x \in \Phi(\omega) .
$$

The Lipschitz constant of $\phi$ is defined as the infimum among all positive $\alpha>0$ for which (3.4) is satisfied.

The main result of this section is the equivalence between the notion of $X$ Lipschitz surfaces and that of intrinsic Lipschitz graphs in the setting of Carnot groups. To this end, we will need the following preliminary result.

Lemma 3.1. For any $\alpha>0$ there exists a Lipschitz function $f_{\alpha}: \mathbb{G} \rightarrow \mathbb{R}$ such that

$$
X_{1} f_{\alpha} \geqslant 1 / 2 \mathcal{L}^{n} \text {-a.e. on } \mathbb{G} \text { and } \partial \mathcal{C}_{\alpha}^{+}=\left\{x \in \mathbb{G}: x_{1}=\alpha\left\|x_{\mathbb{W}}\right\|_{\mathbb{G}}\right\}=\left\{f_{\alpha}=0\right\} \text {. }
$$

In particular, $\partial \mathcal{C}_{\alpha}^{+}$is an X-Lipschitz surface. 
Proof. Let us define

$$
f_{\alpha}(x):= \begin{cases}x_{1}-\alpha\left\|x_{\mathbb{W}}\right\|_{\mathbb{G}} & \text { if }\left|x_{1}\right| \leqslant 2 \alpha\left\|x_{\mathbb{W}}\right\|_{\mathbb{G}} \\ x_{1} / 2 & \text { if } x_{1}>2 \alpha\left\|x_{\mathbb{W}}\right\|_{\mathbb{G}} \\ 3 x_{1} / 2 & \text { if } x_{1}<-2 \alpha\left\|x_{\mathbb{W}}\right\|_{\mathbb{G}} .\end{cases}
$$

The function $f_{\alpha}$ is continuous and $\partial \mathcal{C}_{\alpha}^{+}=\left\{f_{\alpha}=0\right\}$. Since $\mathcal{L}^{n}\left(\partial \mathcal{C}_{2 \alpha}\right)=0$, by the continuity of $f_{\alpha}$ it is enough to show that

$X_{1} f_{\alpha} \geqslant 1 / 2 \quad$ and $\quad\left|X f_{\alpha}\right| \leqslant C \quad$ on $\mathbb{G} \backslash \partial \mathcal{C}_{2 \alpha}=\left\{x \in \mathbb{G}:\left|x_{1}\right| \neq 2 \alpha\left\|x_{\mathbb{W}}\right\|_{\mathbb{G}}\right\}$.

It is easily seen that

$$
\begin{aligned}
& X f_{\alpha}=(1 / 2,0, \ldots, 0) \text { if } x_{1}>2 \alpha\left\|x_{\mathbb{W}}\right\|_{\mathbb{G}} \\
& X f_{\alpha}=(3 / 2,0, \ldots, 0) \text { if } x_{1}<-2 \alpha\left\|x_{\mathbb{W}}\right\|_{\mathbb{G}} .
\end{aligned}
$$

Moreover, we have

$$
x_{\mathbb{W}}=\left(x \cdot h e_{1}\right)_{\mathbb{W}}=\left(\exp \left(h X_{1}\right)(x)\right)_{\mathbb{W}} \quad \text { for any } x \in \mathbb{G}, h \in \mathbb{R},
$$

thus the map $x \mapsto\left\|x_{\mathbb{W}}\right\|_{\mathbb{G}}$ is constant along integral lines of $X_{1}$. In particular

$$
X_{1} f_{\alpha}(x)=1 \quad \text { if }\left|x_{1}\right|<2 \alpha\left\|x_{\mathbb{W}}\right\|_{\mathbb{G}} .
$$

Defining $g: \mathbb{G} \rightarrow \mathbb{R}$ as $g(x):=x_{1}-\alpha\left\|x_{\mathbb{W}}\right\|_{\mathbb{G}}$, we are only left to show that

$$
|X g| \leqslant C \quad \text { on }\left\{x \in \mathbb{G}:\left|x_{1}\right|<2 \alpha\left\|x_{\mathbb{W}}\right\|_{\mathbb{G}}\right\} \text {. }
$$

Taking into account that $x \mapsto x_{\mathbb{W}}$ is smooth, that $\|\cdot\|_{\mathbb{G}}$ is of class $C^{1}$ on $\mathbb{G} \backslash\{0\}$ and that

$$
x_{\mathbb{W}}=0 \Leftrightarrow x \in L:=\left\{\left(x=\left(x_{1}, x^{\prime}\right) \in \mathbb{R} \times \mathbb{R}^{n-1} \equiv \mathbb{G}: x^{\prime}=0\right\},\right.
$$

we get that $g$ is of class $C^{1}$ on $\mathbb{G} \backslash L$. Moreover, $g$ is 1 -homogeneous, thus $X g$ is 0 -homogeneous (i.e., invariant under dilations) and continuous on $\mathbb{G} \backslash L$. Inequality (3.5) will follow if we prove that

$$
|X g| \leqslant C \quad \text { on } \partial B(0,1) \cap\left\{x \in \mathbb{G}:\left|x_{1}\right| \leqslant 2 \alpha\left\|x_{\mathbb{W}}\right\|_{\mathbb{G}}\right\} .
$$

The sets $L$ and $\partial B(0,1) \cap\left\{x \in \mathbb{G}:\left|x_{1}\right| \leqslant 2 \alpha\left\|x_{\mathbb{W}}\right\|_{\mathbb{G}}\right\}$ are compact and disjoint, thus they have positive distance and in particular

$$
\sup \left\{|X g(x)|: x \in \partial B(0,1),\left|x_{1}\right| \leqslant 2 \alpha\left\|x_{\mathbb{W}}\right\|_{\mathbb{G}}\right\}<+\infty
$$

which allows to conclude.

We can now prove the main result of this section. 
Theorem 3.2. A set $S \subset \mathbb{G}$ is an $X$-Lipschitz surface if and only if $S$ is locally the intrinsic graph of an intrinsic Lipschitz function defined on an open subset of a maximal subgroup.

Theorem 3.2 is an easy consequence of the following Proposition 3.3 or, more precisely, of the fact that the latter could be stated also "replacing" $X_{1}$ with a generic $X_{j}, j=2, \ldots, m$. Namely, one could prove that, if $S=\{f=0\}$ is the level set of a Lipschitz function $f: U \subset \mathbb{G} \rightarrow \mathbb{R}$ with $U$ open and $X_{j} f \geqslant l>0$, then $S$ is locally an intrinsic Lipschitz graph (defined on an open subset) along $X_{j}$. Viceversa, if $S$ is an intrinsic Lipschitz graph (defined on an open subset) along $X_{j}$, then $S$ is locally the level set of a Lipschitz function $f: U \subset \mathbb{G} \rightarrow \mathbb{R}$ with $U$ open and $X_{j} f \geqslant l>0$.

Given $I \subset \mathbb{W}$ and $J \subset \mathbb{R}$, we adopt from now on the compact notation $I \cdot J$ to denote the set $\left\{p \cdot q e_{1} \in \mathbb{G}: p \in I, q \in J\right\}$.

Proposition 3.3. Let $S \subset \mathbb{G}$. The following two statements are equivalent:

(i) for any $\bar{x} \in S$ there exist an open neighbourhood $U \subset \mathbb{G}$, a Lipschitz function $f: U \rightarrow \mathbb{R}$ and $l>0$ such that $S \cap U=\{f=0\}$ and $X_{1} f \geqslant l \mathcal{L}^{n}$-a.e. on $U$;

(ii) for any $\bar{x} \in S$ there exist an open set $\omega \subset \mathbb{W}, a, b \in \mathbb{R}$ and an intrinsic Lipschitz map $\phi: \omega \rightarrow(a, b)$ such that $\bar{x} \in U:=\omega \cdot(a, b)$ and $S \cap U=$ $\Phi(\omega)$, where $\Phi$ is defined as in (3.3).

Proof. Step 1. (i) $\Rightarrow$ (ii).

Let $\bar{x} \in S$ be fixed; up to a left translation, we may assume that $\bar{x}=0$. Up to a localization argument we can suppose that $U$ is of the form $U=\omega \cdot(-a, a)$ for suitable $a>0$ and $\omega \subset \mathbb{W}$ open with $0 \in \omega$; we can also assume that $f$ is continuous on $\bar{U}=\bar{\omega} \cdot[-a, a]$. Since $f(0)=0$, reasoning as in Lemma 2.2 we have

$$
f\left(a e_{1}\right)=\exp \left(a X_{1}\right)(0) \geqslant a l>0, \quad f\left(-a e_{1}\right)=\exp \left(-a X_{1}\right)(0) \leqslant-a l<0 .
$$

Therefore, by the continuity of $f$ we may assume that $\omega$ is such that

$$
f\left(y \cdot a e_{1}\right)>0 \text { and } f\left(y \cdot-a e_{1}\right)<0 \quad \text { for any } y \in \omega .
$$

This implies that for any $y \in \omega$ there exists $s_{y} \in(-a, a)$ such that $f\left(y \cdot s_{y} e_{1}\right)=$ $\exp \left(s_{y} X_{1}\right)(y)=0$. Such $s_{y}$ is unique by Lemma 2.2 and we can define $\phi: \omega \rightarrow$ $(-a, a)$ by $\phi(y):=s_{y}$. Clearly, $S \cap U=\Phi(\omega)$ where $\Phi: \omega \rightarrow \mathbb{G}$ is defined as in (3.3).

We claim that $\phi$ is intrinsic Lipschitz with Lipschitz constant not greater than $\alpha:=2 \frac{c L}{l}>0$, where $L$ is the Lipschitz constant of $f$ and $c>0$ is as in (3.2).

Let $x \in \Phi(\omega)$ and $x^{\prime} \in x \cdot \overline{\mathcal{C}_{\alpha}}$ with $x^{\prime} \neq x$ : we have to show that $x^{\prime} \notin \Phi(\omega)=$ $S \cap U$. If $x^{\prime} \notin U$ there is nothing to prove; if instead $x^{\prime} \in U \cap x \cdot \overline{\mathcal{C}_{\alpha}}$, we need 
to show that $f\left(x^{\prime}\right) \neq 0$. We have $x^{\prime}=x \cdot p_{\mathbb{W}} \cdot p_{1} e_{1}$ for some $p_{1}, p_{\mathbb{W}}$ such that $\left|p_{1}\right| \geqslant \alpha\left\|p_{\mathbb{W}}\right\|_{\mathbb{G}}$. If $p_{1}>0$, by Lemma 2.2 we get

$f\left(x^{\prime}\right) \geqslant f\left(x \cdot p_{\mathbb{W}}\right)+l p_{1} \geqslant f(x)-L d\left(x \cdot p_{\mathbb{W}}, x\right)+l p_{1} \geqslant-L c\left\|p_{\mathbb{W}}\right\|_{\mathbb{G}}+l p_{1}>0$,

where we have used the Lipschitz continuity of $f$ and the fact that $f(x)=0$. Notice also that Lemma 2.2 could be applied because $x \cdot p_{\mathbb{W}} \cdot h e_{1} \in U$ for any $h \in\left[0, p_{1}\right]$, which in turn is due to

$$
\begin{aligned}
& \left(x \cdot p_{\mathbb{W}} \cdot h e_{1}\right)_{\mathbb{W}}=\left(x \cdot p_{\mathbb{W}} \cdot p_{1} e_{1}\right)_{\mathbb{W}} \in \omega \\
& \left(x \cdot p_{\mathbb{W}} \cdot h e_{1}\right)_{1}=x_{1}+h \in\left(x_{1}, p_{1}\right) \subset(-a, a) .
\end{aligned}
$$

Similarly, if $p_{1}<0$ we have

$$
f\left(x^{\prime}\right) \leqslant f\left(x \cdot p_{\mathbb{W}}\right)-l\left|p_{1}\right| \leqslant f(x)+L d\left(x \cdot p_{\mathbb{W}}, x\right)-l\left|p_{1}\right| \leqslant L c\left\|p_{\mathbb{W}}\right\|_{\mathbb{G}}-l\left|p_{1}\right|<0
$$

and the claim follows. Notice that the Lipschitz constant of $\phi$ depends only on $l$ and the Lipschitz constant $L$ of $f$.

Step 2. (ii) $\Rightarrow$ (i). Fix $\alpha>0$ such that (3.4) holds and let $f_{\alpha}$ be as in Lemma 3.1. Given $y \in \mathbb{G}$ let us introduce $f_{\alpha, y}(x):=f_{\alpha}\left(y^{-1} \cdot x\right)$; in this way $y \cdot \partial \mathcal{C}_{\alpha}^{+}=\left\{f_{\alpha, y}=\right.$ $0\}$. We claim that the map $f: \mathbb{G} \rightarrow \mathbb{R}$ defined by

$$
f(x):=\sup _{y \in \Phi(\omega)} f_{\alpha, y}(x)
$$

is Lipschitz continuous with $X_{1} f \geqslant 1 / 2 \mathcal{L}^{n}$-a.e. and $\Phi(\omega)=\{f=0\} \cap U$. This would be enough to conclude.

Let us prove our claim. The maps $f_{\alpha, y}$ are uniformly Lipschitz continuous, so $f$ shares the same Lipschitz continuity. For fixed $x \in \mathbb{G}$ and $\epsilon>0$ let $y \in \Phi(\omega)$ be such that

$$
f_{\alpha, y}(x) \geqslant f(x)-\epsilon
$$

since $X_{1} f_{\alpha, y} \geqslant 1 / 2$, we have for any $h \geqslant 0$

$$
f\left(x \cdot h e_{1}\right) \geqslant f_{\alpha, y}\left(x \cdot h e_{1}\right) \geqslant f_{\alpha, y}(x)+h / 2 \geqslant f(x)-\epsilon+h / 2
$$

whence $f\left(x \cdot h e_{1}\right) \geqslant f(x)+h / 2$ for any $x \in \mathbb{G}$ and $h \geqslant 0$. This implies that $X_{1} f(x) \geqslant 1 / 2$ for $\mathcal{L}^{n}$-a.e. $x \in \mathbb{G}$.

Let us prove that $\Phi(\omega) \subset\{f=0\} \cap U$. Let $x \in \Phi(\omega) \subset U$ be fixed. For any $y \in \Phi(\omega), y \neq x$, we have $x \notin y \cdot \overline{\mathcal{C}_{\alpha}^{+}}$and so $f_{\alpha, y}(x)<0$. Since $f_{\alpha, x}(x)=0$ we obtain by definition $f(x)=0$, as claimed.

Finally, we prove that $\{f=0\} \cap U \subset \Phi(\omega)$ by showing that, if $x \in U \backslash \Phi(\omega)$, then $f(x) \neq 0$. Notice that, if $x \in U \backslash \Phi(\omega)$, then $\pi_{\mathbb{W}}(x) \in \omega$ and $x_{1} \neq \phi\left(\pi_{\mathbb{W}}(x)\right)$. The conclusion easily follows from Lemma 2.2 and the fact that $X_{1} f \geqslant 1 / 2$ : indeed, if $x_{1}>\phi\left(\pi_{\mathbb{W}}(x)\right)$ we obtain

$$
\begin{aligned}
f(x) & =f\left(\exp \left(\left(x_{1}-\phi\left(\pi_{\mathbb{W}}(x)\right)\right) X_{1}\right)\left(\Phi\left(\pi_{\mathbb{W}}(x)\right)\right)\right) \\
& \geqslant f\left(\Phi\left(\pi_{\mathbb{W}}(x)\right)\right)+\left(x_{1}-\phi\left(\pi_{\mathbb{W}}(x)\right)\right) / 2=\left(x_{1}-\phi\left(\pi_{\mathbb{W}}(x)\right)\right) / 2>0
\end{aligned}
$$


while if $x_{1}<\phi(\pi \mathbb{W}(x))$

$$
\begin{aligned}
f(x) & =f\left(\exp \left(\left(x_{1}-\phi\left(\pi_{\mathbb{W}}(x)\right)\right) X_{1}\right)\left(\Phi\left(\pi_{\mathbb{W}}(x)\right)\right)\right) \\
& \leqslant f\left(\Phi\left(\pi_{\mathbb{W}}(x)\right)\right)-\left|x_{1}-\phi\left(\pi_{\mathbb{W}}(x)\right)\right| / 2=-\left|x_{1}-\phi\left(\pi_{\mathbb{W}}(x)\right)\right| / 2<0 .
\end{aligned}
$$

This concludes the proof.

We conclude this section by showing an extension result for intrinsic Lipschitz graphs; in the Heisenberg group setting this result has already been proved, with a similar technique, in [38].

Proposition 3.4. Let $\phi: \omega \rightarrow \mathbb{R}$ be an intrinsic Lipschitz function defined on a subset $\omega \subset \mathbb{W}$; assume that $\alpha>0$ is such that (3.4) holds. Then there exists an intrinsic Lipschitz map $\bar{\phi}: \mathbb{W} \rightarrow \mathbb{R}$ such that $\bar{\phi}_{\mid \omega}=\phi$. Moreover, the Lipschitz constant of $\bar{\phi}$ is not greater than a suitable $\beta=\beta(\alpha)$.

Proof. The proof is essentially contained in the proof of Proposition 3.3. For $y \in \mathbb{G}$ and $f_{\alpha}$ as in Lemma 3.1 define $f_{\alpha, y}(x):=f_{\alpha}\left(y^{-1} \cdot x\right)$ and

$$
f(x):=\sup _{y \in \Phi(\omega)} f_{\alpha, y}(x) .
$$

As before, it is possible to prove that $f$ is Lipschitz (with Lipschitz constant depending only on $\alpha$ ) and such that $X_{1} f \geqslant 1 / 2$ and $\Phi(\omega) \subset\{f=0\}$.

Reasoning as in the proof of Proposition 3.3, Step 1, it is not difficult to check that $\{f=0\}$ is the intrinsic graph of a map $\bar{\phi}: \mathbb{W} \rightarrow \mathbb{R}$ with $\bar{\phi}_{\mid \omega}=\phi$. As we noticed, the Lipschitz constant of $\bar{\phi}$ can be controlled in terms of $l=1 / 2$ and the Lipschitz constant of $f$, i.e., in terms of $\alpha$.

Remark 3.5. With the same notation of Proposition 3.4 and its proof: the intrinsic subgraph of $\bar{\phi}$

$$
E_{\bar{\phi}}:=\left\{y \cdot s e_{1}: y \in \omega, s<\phi(y)\right\}
$$

is an $X$-Lipschitz domain. Just check that $E_{\bar{\phi}}=\{f<0\}$ for $f$ as in (3.6).

\section{4. $X$-Lipschitz domains and $X$-perimeter}

\section{1. $X$-perimeter of $X$-Lipschitz domains}

We begin this section by proving an implicit function theorem for $X$-Lipschitz surfaces. As already said in the Introduction, this result is inspired to [22, Theorem $1.1]$.

Proposition 4.1 (implicit function theorem for $X$-Lipschitz surfaces). Let $S$ be an $X$-Lipschitz surface given as level set, $S=\{f=0\}$, of a Lipschitz function $f: U \rightarrow \mathbb{R}, U \subset \mathbb{R}^{n}$ open, with

$$
X_{j} f \geqslant l \quad \mathcal{L}^{n} \text {-a.e. on } U
$$

for suitable $j \in\{1, \ldots, m\}$ and $l>0$. Then, for any $x \in S$ there exist 
- an open neighbourhood $\mathcal{Q} \subset U$ of $x$;

- an open bounded domain $I \subset \mathbb{R}^{n-1}$ with $0 \in I$;

- a positive real number a;

- a diffeomorphism $G: I \times(-a, a) \rightarrow \mathcal{Q}$ with $G(0)=x$;

- a continuous function $\phi: I \rightarrow(-a / 2, a / 2)$ with $\phi(0)=0$

such that $d G^{-1}\left[X_{j}\right]=\partial_{s_{n}}$, where $\left(s_{1}, \ldots, s_{n}\right)$ are the coordinates of points in $I \times(-a, a) \subset \mathbb{R}^{n-1} \times \mathbb{R}$, and

$$
G^{-1}(S \cap \mathcal{Q})=\{(y, \phi(y)): y \in I\} .
$$

Proof. We complete the family $X$ to a system $X_{1}, \ldots, X_{n}$ of vector fields that forms a basis of $\mathbb{R}^{n}$ in a neighbourhood $U^{\prime} \subset U$ of $x$. Define

$G\left(s_{1}, \ldots, s_{n}\right):=\exp \left(s_{n} X_{j}\right) \exp \left(s_{1} X_{1}+\cdots+s_{j-1} X_{j-1}+s_{j} X_{j+1}+\cdots+s_{n-1} X_{n}\right)(x)$.

The map $G$ is a diffeomorphism from some neighbourhood $V \subset \mathbb{R}^{n}$ of the origin to some neighbourhood $U^{\prime \prime} \subset U^{\prime}$ of $x$; by definition, $G(0)=x$ and $X_{j}=d G\left[\partial_{s_{n}}\right]$. We may assume that $V=I \times(-a, a)$ for some $I$ and $a$ as in the statement. Set $\mathcal{Q}:=G(V)$.

Let us define the continuous map $\tilde{f}:=f \circ G: V \rightarrow \mathbb{R}$; we have $\tilde{f}(0)=$ $f(x)=0$ and $\partial_{s_{n}} \tilde{f}=\left(X_{j} f\right) \circ G \geqslant l>0$ a.e. on $V$. By Lemma 2.2 we have

$$
\tilde{f}(0, \ldots, 0, a / 2) \geqslant a l / 2>0 \quad \text { and } \quad \tilde{f}(0, \ldots, 0,-a / 2) \leqslant-a l / 2<0 .
$$

Possibly restricting $I$, by the continuity of $\tilde{f}$ we may assume that

$$
\tilde{f}(y,-a / 2)<0<\tilde{f}(y, a / 2) \text { for any } y \in I .
$$

By the continuity of $\tilde{f}$ and Lemma 2.2, for any $y \in I$ there exists a unique $\phi(y) \in$ $(-a / 2, a / 2)$ such that $\tilde{f}(y, \phi(y))=0$. This gives (4.1).

Finally, the continuity of $\phi$ follows from that of $\tilde{f}$ : indeed, for any $y \in I$ and $\epsilon>0$ we have by Lemma 2.2

$$
\tilde{f}(y, \phi(y)-\epsilon) \leqslant-\epsilon l<0<\epsilon l \leqslant \tilde{f}(y, \phi(y)+\epsilon)
$$

thus there exists $\delta>0$ such that

$$
\tilde{f}\left(y^{\prime}, \phi(y)-\epsilon\right)<0<\tilde{f}\left(y^{\prime}, \phi(y)+\epsilon\right) \text { for any } y^{\prime} \in I \text { with }\left|y-y^{\prime}\right|<\delta .
$$

This gives $\phi(y)-\epsilon<\phi\left(y^{\prime}\right)<\phi(y)+\epsilon$ for any such $y^{\prime}$ and proves the continuity of $\phi$.

For $X$-regular surfaces, the parameterizing map $\phi$ was introduced in [33] (in the setting of the Heisenberg group), [35] (in Carnot groups) and [22] (in the general framework of CC spaces). The problem of the optimal regularity of $\phi$ is a delicate matter even for $X$-regular surfaces, see [3,12,13,22].

A first consequence of Proposition 4.1 is the fact that $X$-Lipschitz surfaces have null Lebesgue measure. 
Corollary 4.2. Let $S$ be as in Proposition 4.1; then $\mathcal{L}^{n}(S)=0$. In particular, any $X$-Lipschitz surface has null Lebesgue measure.

Proof. It is enough to prove that for any $x \in S$ there exists a neighbourhood $\mathcal{Q}$ such that $\mathcal{L}^{n}(S \cap \mathcal{Q})=0$. Let then $x \in S$ be fixed and $\mathcal{Q}$ and $G$ be given by Proposition 4.1. By (4.1) we have $\mathcal{L}^{n}\left(G^{-1}(S \cap \mathcal{Q})\right)=0$ and this gives $\mathcal{L}^{n}(S \cap \mathcal{Q})=0$.

The following two technical results will be extensively used in the sequel: roughly speaking, they allow to choose the defining function of $X$-Lipschitz or $X$-regular domains "as smooth as possible". As already done in the proof of Proposition 4.1, we hereafter agree to use a tilde to denote functions, sets, etc., when they are considered not in the CC space $\mathbb{R}^{n}$ but in the coordinates given by Proposition 4.1.

Lemma 4.3. An open set $\Omega \subset \mathbb{R}^{n}$ is an X-Lipschitz domain if and only if for any $x \in \partial \Omega$ there exist a neighbourhood $U$, a Lipschitz function $f: U \rightarrow \mathbb{R}$ and $j \in\{1, \ldots, m\}$ such that $\Omega \cap U=\{f>0\}$ or $\Omega \cap U=\{f<0\}$ and

$$
f \in C^{\infty}(U \backslash \partial \Omega) \quad \text { and } \quad \inf _{U \backslash \partial \Omega} X_{j} f>0 .
$$

Proof. One implication is clear: if $\Omega$ is such that for any $x \in \partial \Omega$ we can find $U, f, j$ as in the statement, then $\Omega$ is an $X$-Lipschitz domain because of Corollary 4.2.

In order to prove the reverse implication, we assume that $\Omega$ is $X$-Lipschitz. Given $x \in \partial \Omega$ there exist an open neighbourhood $U_{0} \subset \mathbb{R}^{n}$, a Lipschitz function $f_{0}: U_{0} \rightarrow \mathbb{R}, l>0$ and $j \in\{1, \ldots, m\}$ such that

$$
\Omega \cap U_{0}=\left\{f_{0}>0\right\} \text { or } \Omega \cap U_{0}=\left\{f_{0}<0\right\} \quad \text { and } \quad X_{j} f_{0} \geqslant l \mathcal{L}^{n} \text {-a.e. on } U_{0} .
$$

Let $\mathcal{Q}, I, a, G$ be given by Proposition 4.1 (applied to $S=\partial \Omega \cap U$ and with $f_{0}$ and $U_{0}$ in place, respectively, of $f$ and $\left.U\right)$. Set $\tilde{f}_{0}:=f_{0} \circ G: I \times(-a, a) \rightarrow \mathbb{R}$ and $W_{k}:=\left\{\left|\tilde{f}_{0}\right|>1 / k\right\}, k \geqslant 1$, so that

$$
W_{k} \uparrow\left\{\tilde{f}_{0} \neq 0\right\}=(I \times(-a, a)) \backslash G^{-1}(\partial \Omega \cap \mathcal{Q}) .
$$

Let $I^{\prime} \Subset I$ be a fixed open set and define $V:=I^{\prime} \times(-a / 2, a / 2)$. One can fix functions $\eta_{k} \in C_{c}^{\infty}\left(W_{k+2} \backslash \bar{W}_{k}\right)$ such that

$$
0 \leqslant \eta_{k} \leqslant 1 \quad \text { and } \quad \sum_{k=0}^{\infty} \eta_{k}=1 \text { on } V \cap\left\{\tilde{f}_{0} \neq 0\right\} .
$$

We fix a smooth mollification kernel $K$ supported in the Euclidean unit ball $\mathscr{B}(0,1) \subset \mathbb{R}^{n}$ and set $K_{\epsilon}(z):=\epsilon^{-n} K(z / \epsilon)$. If $\epsilon_{k}$ is smaller than the Euclidean distance between spt $\eta_{k}$ and $\partial\left(W_{k+2} \backslash \bar{W}_{k}\right)$, then the function $u_{k}: I \times(-a, a) \rightarrow \mathbb{R}$ defined by

$u_{k}(s):=\left(\eta_{k} \tilde{f}_{0}\right) * K_{\epsilon_{k}}(s)$ if the Euclidean distance between $s$ and spt $\eta_{k}$ is less than $\epsilon_{k}$ $u_{k}=0$ otherwise 
is of class $C^{\infty}$. We define $\tilde{f}: V \rightarrow \mathbb{R}$ by

$$
\tilde{f}:= \begin{cases}\sum_{k=0}^{\infty} u_{k} & \text { on } V \cap\left\{\tilde{f}_{0} \neq 0\right\} \\ 0 & \text { on } V \cap\left\{\tilde{f}_{0}=0\right\}=V \cap G^{-1}(\partial \Omega \cap \mathcal{Q}) .\end{cases}
$$

Notice that the summation is locally finite, whence $\tilde{f}$ is of class $C^{\infty}$ on $V \cap\left\{\tilde{f}_{0} \neq\right.$ $0\}$. We claim that, possibly restricting $\epsilon_{k}, I^{\prime}$ and $a$, the statement of the lemma is fulfilled by $U:=G(V)$ and $f:=\tilde{f} \circ G^{-1}: U \rightarrow \mathbb{R}$. To begin with, the $C^{\infty}$-smoothness of $f$ on $U \backslash \partial \Omega$ is straightforward.

Step 1. $\Omega \cap U=\{f>0\}$ or $\Omega \cap U=\{f<0\}$.

It will be enough to show that

$$
V \cap\left\{\tilde{f}_{0}>0\right\}=V \cap\{\tilde{f}>0\} \quad \text { and } \quad V \cap\left\{\tilde{f}_{0}<0\right\}=V \cap\{\tilde{f}<0\} .
$$

Notice that $W_{k}$ is the disjoint union of the two open sets $W_{k}^{+}:=\left\{\tilde{f}_{0}>1 / k\right\}$ and $W_{k}^{-}:=\left\{\tilde{f}_{0}<-1 / k\right\}$. Thus we have

$$
W_{k+2} \backslash \bar{W}_{k}=\left(W_{k+2}^{+} \backslash \bar{W}_{k}^{+}\right) \cup\left(W_{k+2}^{-} \backslash \bar{W}_{k}^{-}\right)
$$

and our choice of $\epsilon_{k}$ gives

$$
\begin{aligned}
& \text { if } s \in W_{k+2}^{+} \backslash \bar{W}_{k}^{+} \text {, then } u_{k} \geqslant 0 \\
& \text { if } s \in W_{k+2}^{-} \backslash \bar{W}_{k}^{-} \text {, then } u_{k} \leqslant 0 .
\end{aligned}
$$

This implies that

$$
\tilde{f} \geqslant 0 \text { on }\left\{\tilde{f}_{0}>0\right\} \quad \text { and } \quad \tilde{f} \leqslant 0 \text { on }\left\{\tilde{f}_{0}<0\right\} .
$$

Due to (4.2), for any $s \in V \backslash\left\{\tilde{f}_{0}=0\right\}$ there exists $\bar{k} \geqslant 1$ such that $\eta_{\bar{k}}(s)>0$. This gives $s \in V \cap W_{\bar{k}+2} \backslash \bar{W}_{\bar{k}}$ and

$$
\begin{aligned}
& u_{\bar{k}}(s)>0 \text { if } s \in V \cap W_{\bar{k}+2}^{+} \backslash \bar{W}_{\bar{k}}^{+} \\
& u_{\bar{k}}(s)<0 \text { if } s \in V \cap W_{\bar{k}+2}^{-} \backslash \bar{W}_{\bar{k}}^{-} .
\end{aligned}
$$

By (4.4) and (4.5), if $s \in V$ we have

$$
\tilde{f}_{0}(s)>0 \Leftrightarrow \tilde{f}(s)>0 \quad \text { and } \quad \tilde{f}_{0}(s)<0 \Leftrightarrow \tilde{f}(s)<0
$$

which is (4.3).

Step 2. $f$ is continuous on $U$.

It is enough to show that $\tilde{f}$ is continuous on $V$. Since $\tilde{f}$ is clearly continuous on $\left\{\tilde{f}_{0} \neq 0\right\}$, it suffices to show that for any $s_{0} \in V \cap\left\{\tilde{f}_{0}=0\right\}=V \cap G^{-1}(\partial \Omega \cap \mathcal{Q})$

$$
\lim _{\substack{s \rightarrow s_{0} \\ s \in V \cap\{\tilde{f} \neq 0\}}} \tilde{f}(s)=0=\tilde{f}\left(s_{0}\right) .
$$


If $s_{\ell} \rightarrow s_{0}, \quad\left(s_{\ell}\right)_{\ell} \subset V \cap\left\{\tilde{f}_{0} \neq 0\right\}$, we have $s_{\ell} \in W_{k_{\ell}+1} \backslash \overline{W_{k_{\ell}}}$ with $k_{\ell} \rightarrow \infty$; therefore, it will be enough to show that

$$
|\tilde{f}| \leqslant \frac{1}{k}+\frac{1}{k-1} \quad \text { on } W_{k+1} \backslash \overline{W_{k}} .
$$

Since spt $\eta_{k} \subset W_{k+2} \backslash \bar{W}_{k}$ we have

$$
\left|u_{k}(s)\right| \leqslant \int_{\mathscr{B}\left(0, \epsilon_{k}\right)} \eta_{k}(s-h)\left|\tilde{f}_{0}(s-h)\right| K_{\epsilon_{k}}(h) d \mathcal{L}^{n}(h) \leqslant \frac{1}{k} .
$$

Inequality (4.6) follows because $\tilde{f}=u_{k}+u_{k-1}$ on $W_{k+1} \backslash \overline{W_{k}}$.

Step 3. $f$ is Lipschitz on $U$.

We claim that, if each $\epsilon_{\underline{k}}$ is small enough, the $\underset{\widetilde{X}}{\tilde{X}} \tilde{f} \mid \tilde{\sim}_{\mathcal{X}}$ is bounded on $V$, where for any $i=1, \ldots, m$ we set $\widetilde{X}_{i}:=d G^{-1}\left[X_{i}\right]$ and $\widetilde{X}:=\left(\widetilde{X}_{1}, \ldots, \widetilde{X}_{m}\right)$. This would imply that $|X f|$ is uniformly bounded on $U$ and, in turn, that $f$ is locally Lipschitz continuous on $U$. The Lipschitz continuity of $f$ on $U$ follows up to restricting $I$ and $a$.

Write $\widetilde{X}_{i}=\sum_{\ell=1}^{n} b_{\ell}^{i} \partial_{s_{\ell}}$ for suitable smooth coefficients $b_{\ell}^{i}: I \times(-a, a) \rightarrow \mathbb{R}$. For any $k \in \mathbb{N}$ and $i=1, \ldots, m$ it is (see also [40, Lemma 2.6] and [32])

$$
\tilde{X}_{i} u_{k}=\left(\tilde{X}_{i}\left(\eta_{k} \tilde{f}_{0}\right)\right) * K_{\epsilon_{k}}+R_{k}^{i} \quad \text { on } V
$$

where for $s \in V$ we have defined

$$
R_{k}^{i}(s):=\frac{1}{\epsilon_{k}} \int_{\mathbb{R}^{n}}\left[\eta_{k}\left(s+\epsilon_{k} h\right) \tilde{f}_{0}\left(s+\epsilon_{k} h\right) \sum_{\ell=1}^{n} \frac{\partial B_{\ell k}^{i}}{\partial h_{\ell}}(s, h)\right] d h
$$

and $B_{\ell k}^{i}(s, h):=\left(b_{\ell}^{i}\left(s+\epsilon_{k} h\right)-b_{\ell}^{i}(s)\right) K(h)$. We have

$$
\begin{aligned}
\frac{\partial B_{\ell k}^{i}}{\partial h_{\ell}}(s, h) & =\epsilon_{k} \frac{\partial b_{\ell}^{i}}{\partial s_{\ell}}\left(s+\epsilon_{k} h\right) K(h)+\left(b_{\ell}^{i}\left(s+\epsilon_{k} h\right)-b_{\ell}^{i}(s)\right) \frac{\partial K}{\partial h_{\ell}}(h) \\
& =\epsilon_{k}\left[\frac{\partial b_{\ell}^{i}}{\partial s_{\ell}}(s) K(h)+\left\langle\nabla b_{\ell}^{i}(s), h\right\rangle \frac{\partial K}{\partial h_{\ell}}(h)+O\left(\epsilon_{k}\right)\right]
\end{aligned}
$$

thus

$$
\begin{aligned}
R_{k}^{i}(s)=\int_{\mathbb{R}^{n}} \eta_{k}\left(s+\epsilon_{k} h\right) \tilde{f}_{0}\left(s+\epsilon_{k} h\right) \sum_{\ell=1}^{n} & {\left[\frac{\partial b_{\ell}^{i}}{\partial s_{\ell}}(s) K(h)\right.} \\
& \left.+\left\langle\nabla b_{\ell}^{i}(s), h\right\rangle \frac{\partial K}{\partial h_{\ell}}(h)+O\left(\epsilon_{k}\right)\right] d h \\
=\eta_{k}(s) \tilde{f}_{0}(s) \int_{\mathscr{B}(0,1)} \sum_{\ell=1}^{n}\left[\frac{\partial b_{\ell}^{i}}{\partial s_{\ell}}(s) K(h)\right. & \left.+\left\langle\nabla b_{\ell}^{i}(s), h\right\rangle \frac{\partial K}{\partial h_{\ell}}(h)\right] d h+o(1)+O\left(\epsilon_{k}\right) \\
=o(1)+O\left(\epsilon_{k}\right) &
\end{aligned}
$$


where the last equality follows because the integral on $\mathscr{B}(0,1)$ is null due to an integration by parts. Notice also that, as $\epsilon_{k} \rightarrow 0$, the quantity $o(1)$ is uniform in $s \in V$ due to the uniform continuity of $\eta_{k} \tilde{f}_{0}$. Therefore, if $\epsilon_{k}$ is small enough we may assume $\left|R_{k}^{i}(s)\right| \leqslant 2^{-k}$ on $V$.

Recall that $u_{k}$ is supported in $W_{k+2} \backslash \bar{W}_{k}$, thus

$\tilde{X}_{i} \tilde{f}=\left(\tilde{X}_{i}\left(\eta_{k} \tilde{f}_{0}\right)\right) * K_{\epsilon_{k}}+R_{k}^{i}+\left(\tilde{X}_{i}\left(\eta_{k+1} \tilde{f}_{0}\right)\right) * K_{\epsilon_{k+1}}+R_{k+1}^{i} \quad$ on $V \cap \bar{W}_{k+2} \backslash W_{k+1}$,

whence

$$
\begin{aligned}
\left|\tilde{X}_{i} \tilde{f}\right| \leqslant & \left|\left(\tilde{X}_{i}\left(\eta_{k} \tilde{f}_{0}\right)\right) * K_{\epsilon_{k}}+\left(\tilde{X}_{i}\left(\eta_{k+1} \tilde{f}_{0}\right)\right) * K_{\epsilon_{k+1}}\right|+2^{1-k} \\
\leqslant & \left|\left(\tilde{f}_{0} \widetilde{X}_{i} \eta_{k}\right) * K_{\epsilon_{k}}+\left(\tilde{f}_{0} \widetilde{X}_{i} \eta_{k+1}\right) * K_{\epsilon_{k+1}}\right| \\
& +\left|\left(\eta_{k} \widetilde{X}_{i} \tilde{f}_{0}\right) * K_{\epsilon_{k}}\right|+\left|\left(\eta_{k+1} \tilde{X}_{i} \tilde{f}_{0}\right) * K_{\epsilon_{k+1}}\right|+2^{1-k} \text { on } V \cap \bar{W}_{k+2} \backslash W_{k+1} .
\end{aligned}
$$

Since $\eta_{k}+\eta_{k+1}=1$ on $V \cap \bar{W}_{k+2} \backslash W_{k+1}$, we have

$$
\left(\tilde{f}_{0} \tilde{X}_{i} \eta_{k}\right) * K_{\epsilon_{k}}+\left(\tilde{f}_{0} \tilde{X}_{i} \eta_{k+1}\right) * K_{\epsilon_{k+1}} \longrightarrow \tilde{f}_{0} \tilde{X}_{i}\left(\eta_{k}+\eta_{k+1}\right)=0
$$

uniformly on $V \cap \bar{W}_{k+2} \backslash W_{k+1}$ as $\epsilon_{k}, \epsilon_{k+1} \rightarrow 0$. Therefore, if $\epsilon_{k}$ and $\epsilon_{k+1}$ are sufficiently small we have

$$
\left|\tilde{X}_{i} \tilde{f}\right| \leqslant 2\left\|\tilde{X}_{i} \tilde{f}_{0}\right\|_{L^{\infty}(V)}+2^{2-k} \leqslant 2\left\|X f_{0}\right\|_{L^{\infty}\left(U_{0}\right)}+2^{2-k} \quad \text { in } V \cap \bar{W}_{k+2} \backslash W_{k+1}
$$

and our claim is proved.

Step 4: $\inf _{U \backslash \partial \Omega} X_{j} f>0$.

It is enough to estimate $\tilde{X}_{j} \tilde{f}$ from below on $V \backslash G^{-1}(\partial \Omega \cap \mathcal{Q})=V \cap\left\{\tilde{f}_{0} \neq 0\right\}$; notice that $R_{k}^{j}=0$ for any $k$ because $\tilde{X}_{j}=(0, \ldots, 0,1)$. We have

$\tilde{X}_{j} \tilde{f}=\partial_{s_{n}} \tilde{f}=\sum_{k=0}^{\infty}\left(\tilde{f}_{0} \partial_{s_{n}} \eta_{k}\right) * K_{\epsilon_{k}}+\sum_{k=0}^{\infty}\left(\eta_{k} \partial_{s_{n}} \tilde{f}_{0}\right) * K_{\epsilon_{k}} \quad$ on $V \cap\left\{\tilde{f}_{0} \neq 0\right\}$.

Reasoning as in (4.8) we may choose each $\epsilon_{k}$ so small that for any $k$

$$
\left|\left(\tilde{f}_{0} \partial_{s_{n}} \eta_{k}\right) * K_{\epsilon_{k}}+\left(\tilde{f}_{0} \partial_{s_{n}} \eta_{k+1}\right) * K_{\epsilon_{k+1}}\right| \leqslant l / 4 .
$$

Since for any $k$

$\left|\sum_{k=0}^{\infty}\left(\tilde{f}_{0} \partial_{s_{n}} \eta_{k}\right) * K_{\epsilon_{k}}\right|=\left|\left(\tilde{f}_{0} \partial_{s_{n}} \eta_{k}\right) * K_{\epsilon_{k}}+\left(\tilde{f}_{0} \partial_{s_{n}} \eta_{k+1}\right) * K_{\epsilon_{k+1}}\right|$ on $V \cap \bar{W}_{k+2} \backslash W_{k+1}$

we obtain

$$
\begin{aligned}
\tilde{X}_{j} \tilde{f}=\partial_{s_{n}} \tilde{f} & \geqslant \sum_{k=0}^{\infty}\left(\eta_{k} \partial_{s_{n}} \tilde{f}_{0}\right) * K_{\epsilon_{k}}-\left|\sum_{k=0}^{\infty}\left(\tilde{f}_{0} \partial_{s_{n}} \eta_{k}\right) * K_{\epsilon_{k}}\right| \\
& \geqslant l \sum_{k=0}^{\infty}\left(\eta_{k} * K_{\epsilon_{k}}\right)-l / 4 \quad \text { on } V \cap\left\{\tilde{f_{0}} \neq 0\right\} .
\end{aligned}
$$


By (4.2) we have $\sum_{k=0}^{\infty}\left(\eta_{k} * K_{\epsilon_{k}}\right) \geqslant 1 / 2$ provided the $\epsilon_{k}$ 's are small enough, whence $\tilde{X}_{j} \tilde{f} \geqslant l / 4$ on $V \cap\left\{\tilde{f}_{0} \neq 0\right\}$, as desired.

In case $\Omega$ is not only $X$-Lipschitz but also $X$-regular, Lemma 4.3 can be refined as follows.

Lemma 4.4. An open set $\Omega \subset \mathbb{R}^{n}$ is an X-regular domain if and only if for any $x \in \partial \Omega$ there exist a neighbourhood $U$ and a function $f: U \rightarrow \mathbb{R}$ such that $\Omega \cap U=\{f>0\}$ or $\Omega \cap U=\{f<0\}$ and

$$
f \in C_{X}^{1}(U) \cap C^{\infty}(U \backslash \partial \Omega) \text { and } X f \neq 0 \text { on } U .
$$

Proof. As in Lemma 4.3, one implication is clear. For the reverse one, we assume that $\Omega$ is $X$-regular and namely that for any $x \in \partial \Omega$ there exists a neighbourhood $U_{0}$ and $f_{0} \in C_{X}^{1}\left(U_{0}\right)$ such that

$$
\Omega \cap U_{0}=\left\{f_{0}>0\right\} \text { or } \Omega \cap U_{0}=\left\{f_{0}<0\right\} \quad \text { and } \quad X f_{0} \neq 0 \text { on } U_{0} .
$$

Up to restricting $U_{0}$ and possibly changing the sign of $f_{0}$, we may assume that there exists $j \in\{1, \ldots, m\}$ such that $X_{j} f_{0} \geqslant l$ on $U_{0}$ for a suitable $l>0$. We can then follow the proof of Lemma 4.3 and define in the same way

$$
\tilde{f}_{0}, G, \mathcal{Q} \subset U_{0}, \tilde{X}_{i}, \tilde{X}, W_{k}, V, \eta_{k}, \epsilon_{k}, \tilde{f}, f, U
$$

We have only to check that $X f$ is continuous on $U$, i.e., that $\tilde{X} \tilde{f}$ exists and is continuous on $V$. Due to the smoothness of $\tilde{f}$ on $V \backslash G^{-1}(\partial \Omega \cap \mathcal{Q})$, it is enough to prove that for any $s_{0} \in V \cap G^{-1}(\partial \Omega \cap \mathcal{Q})=V \cap\left\{\tilde{f}_{0}=0\right\}$

$$
\lim _{\substack{s \rightarrow s_{0} \\ s \in V \backslash\left\{\tilde{f}_{0}=0\right\}}} \tilde{X} \tilde{f}(s)=\tilde{X} \tilde{f}_{0}\left(s_{0}\right) .
$$

Indeed, this would imply that $\tilde{X} \tilde{f}$ exists at $s_{0}$ and coincides with $\tilde{X} \tilde{f}_{0}\left(s_{0}\right)$; moreover, the continuity of $\widetilde{X} \tilde{f}$ would be now straightforward.

Reasoning as in (4.7) and (4.8), we may assume that the $\epsilon_{k}$ 's are small enough to have for any $i=1, \ldots, m$

$$
\begin{aligned}
& \left|\tilde{X}_{i} \tilde{f}-\tilde{X}_{i} \tilde{f}_{0}\right| \\
\leqslant & \left|\left(\tilde{X}_{i}\left(\eta_{k} \tilde{f}_{0}\right)\right) * K_{\epsilon_{k}}+\left(\tilde{X}_{i}\left(\eta_{k+1} \tilde{f}_{0}\right)\right) * K_{\epsilon_{k+1}}-\tilde{X}_{i} \tilde{f}_{0}\right|+\left|R_{k}^{i}+R_{k+1}^{i}\right| \\
\leqslant & \left|\left(\eta_{k} \tilde{X}_{i} \tilde{f}_{0}\right) * K_{\epsilon_{k}}+\left(\eta_{k+1} \tilde{X}_{i} \tilde{f}_{0}\right) * K_{\epsilon_{k+1}}-\tilde{X}_{i} \tilde{f}_{0}\right| \\
& +\left|\left(\tilde{f}_{0} \tilde{X}_{i} \eta_{k}\right) * K_{\epsilon_{k}}+\left(\tilde{f}_{0} \tilde{X}_{i} \eta_{k+1}\right) * K_{\epsilon_{k+1}}\right|+2^{1-k} \\
\leqslant & \left|\left(\eta_{k} \tilde{X}_{i} \tilde{f}_{0}\right) * K_{\epsilon_{k}}+\left(\eta_{k+1} \tilde{X}_{i} \tilde{f}_{0}\right) * K_{\epsilon_{k+1}}-\tilde{X}_{i} \tilde{f}_{0}\right|+2^{2-k} \quad \text { on } V \cap \bar{W}_{k+2} \backslash W_{k+1} .
\end{aligned}
$$

The continuity of $\tilde{X} \tilde{f}_{0}$ ensures that

$$
\left(\eta_{k} \tilde{X}_{i} \tilde{f}_{0}\right) * K_{\epsilon_{k}}+\left(\eta_{k+1} \tilde{X}_{i} \tilde{f}_{0}\right) * K_{\epsilon_{k+1}} \rightarrow \tilde{X}_{i} \tilde{f}_{0} \quad \text { uniformly on } V \cap \bar{W}_{k+2} \backslash W_{k+1}
$$


as $\epsilon_{k}, \epsilon_{k+1} \rightarrow 0$. Therefore we may assume that

$$
\left|\tilde{X}_{i} \tilde{f}-\tilde{X}_{i} \tilde{f}_{0}\right| \leqslant 2^{3-k} \text { on } V \cap \bar{W}_{k+2} \backslash W_{k+1} .
$$

Using the continuity of $\tilde{X} \tilde{f}_{0}$ and the fact that, if $s_{\ell} \rightarrow s_{0}$ and $s_{\ell} \in V \backslash\left\{\tilde{f}_{0}=0\right\}$ then $s_{\ell} \in V \cap \bar{W}_{k_{\ell}+2} \backslash W_{k_{\ell}+1}$ with $k_{\ell} \rightarrow \infty$, we get that for any $s_{0} \in V \cap G^{-1}(\partial \Omega \cap \mathcal{Q})$ it holds

$$
\lim _{\substack{s \rightarrow s_{0} \\ s \in V \backslash\left\{\tilde{f}_{0}=0\right\}}}\left|\tilde{X} \tilde{f}(s)-\tilde{X} \tilde{f}_{0}\left(s_{0}\right)\right|=0 .
$$

Equality (4.9) follows and the proof is concluded.

We can now prove that $X$-Lipschitz domains have locally finite $X$-perimeter. Theorem 1.2 will follow quite easily from the next result, where we provide an explicit representation formula for the $X$-perimeter in the local coordinates given by Proposition 4.1. Similar formulae have been obtained for $X$-regular surfaces in $[3,22,33,35]$.

Proposition 4.5. Let $S$ be an $X$-Lipschitz surface given as level set $S=\{f=0\}$ of a Lipschitz function $f: U \rightarrow \mathbb{R}, U \subset \mathbb{R}^{n}$ open, such that $f \in C^{\infty}(U \backslash S)$ and

$$
l:=\inf _{U \backslash S} X_{j} f>0 \quad \text { for a suitable } j \in\{1, \ldots, m\} .
$$

Let $x \in S$ and $\Omega:=\{f>0\} \subset U$. Then the open neighbourhood $\mathcal{Q} \subset U$ of $x$ given by Proposition 4.1 can be chosen so that

$$
v_{\Omega}|\partial \Omega|_{X}\left\llcorner\mathcal{Q}=(G \circ \Phi)_{\#}\left(\rho \mathcal{L}^{n-1}\llcorner I)\right.\right.
$$

for a suitable $\rho \in L^{\infty}\left(I, \mathbb{R}^{m}\right)$. Here $I, a, G, \phi$ are as in Proposition 4.1 and $\Phi$ is the map defined by $I \ni y \mapsto \Phi(y):=(y, \phi(y)) \in I \times(-a / 2, a / 2)$.

In particular

$$
|\partial \Omega|_{X}\left\llcorner\mathcal{Q}=(G \circ \Phi)_{\#}\left(|\rho| \mathcal{L}^{n-1}\llcorner I)\right.\right.
$$

and $|\partial \Omega|_{X}(\mathcal{Q})<\infty$.

Proof. Let us define $\tilde{f}:=f \circ G: I \times(-a, a) \rightarrow \mathbb{R}$ and $\widetilde{\Omega}:=G^{-1}(\Omega \cap \mathcal{Q})=$ $\{\tilde{f}>0\}$. We also set

$$
\Omega_{\epsilon}:=\{f>\epsilon\} \quad \text { and } \quad \widetilde{\Omega}_{\epsilon}:=G^{-1}\left(\Omega_{\epsilon} \cap \mathcal{Q}\right)=\{\tilde{f}>\epsilon\} .
$$

Since $\partial_{S_{n}} \tilde{f}=\left(X_{j} f\right) \circ G \geqslant l$, the classical implicit function theorem ensures that (possibly restricting $I$ ) for small enough $\epsilon>0$ there exists a smooth map $\phi_{\epsilon}: I \rightarrow(-a, a)$ such that

$$
\{\tilde{f}=\epsilon\}=\left\{\left(y, \phi_{\epsilon}(y)\right): y \in I\right\} .
$$


Since $\partial_{s_{n}} \tilde{f} \geqslant l>0$ we have that $\widetilde{\Omega}$ and $\widetilde{\Omega}_{\epsilon}$ are the epigraphs of $\phi, \phi_{\epsilon}$ :

$$
\begin{aligned}
& \widetilde{\Omega}=\left\{\left(s_{1}, \ldots, s_{n}\right) \in I \times(-a, a): s_{n}>\phi\left(s_{1}, \ldots, s_{n-1}\right)\right\} \\
& \widetilde{\Omega}_{\epsilon}=\left\{\left(s_{1}, \ldots, s_{n}\right) \in I \times(-a, a): s_{n}>\phi_{\epsilon}\left(s_{1}, \ldots, s_{n-1}\right)\right\} .
\end{aligned}
$$

Moreover, $\phi_{\epsilon}>\phi_{\delta}>0$ for any $\epsilon>\delta>0$. For any $y \in I$ we have by Lemma 2.2

$$
\begin{aligned}
\epsilon & =\tilde{f}\left(y, \phi_{\epsilon}(y)\right)-\tilde{f}(y, \phi(y)) \\
& =\tilde{f}\left(\exp \left(\left(\phi_{\epsilon}(y)-\phi(y)\right) \partial_{s_{n}}\right)(y, \phi(y))\right)-\tilde{f}(y, \phi(y)) \geqslant l\left(\phi_{\epsilon}(y)-\phi(y)\right)>0,
\end{aligned}
$$

whence $\phi_{\epsilon} \rightarrow \phi$ uniformly on $I$.

Let $g \in C_{c}^{1}\left(\mathcal{Q}, \mathbb{R}^{m}\right)$ be fixed; since $\partial \Omega_{\epsilon}$ is smooth we have

$-\int_{\Omega} \operatorname{div}_{X} g d \mathcal{L}^{n}=-\lim _{\epsilon \rightarrow 0} \int_{\Omega_{\epsilon}} \operatorname{div}_{X} g d \mathcal{L}^{n}=\lim _{\epsilon \rightarrow 0} \int_{\partial \Omega_{\epsilon}}\left\langle\boldsymbol{n}_{\Omega_{\epsilon}}, \sum_{k=1}^{m} g_{k} X_{k}\right\rangle d \mathcal{H}^{n-1}$,

where $\boldsymbol{n}_{\Omega_{\epsilon}}$ is the Euclidean inner normal to $\partial \Omega_{\epsilon}$. Taking into account the classical area formula (see e.g. [55]) we obtain

$$
\begin{aligned}
& \int_{\partial \Omega_{\epsilon}}\left\langle\boldsymbol{n}_{\Omega_{\epsilon}}, \sum_{k=1}^{m} g_{k} X_{k}\right\rangle d \mathcal{H}^{n-1}=\int_{\mathcal{Q} \cap \partial \Omega_{\epsilon}}\left\langle\boldsymbol{n}_{\Omega_{\epsilon}}, \sum_{k=1}^{m} g_{k} X_{k}\right\rangle d \mathcal{H}^{n-1} \\
= & \int_{\partial \tilde{\Omega}_{\epsilon}}\left\langle\boldsymbol{n}_{\Omega_{\epsilon}} \circ G,\left(\sum_{k=1}^{m} g_{k} X_{k}\right) \circ G\right\rangle\left|\operatorname{det} J G_{\mid \operatorname{Tan} \partial \widetilde{\Omega}_{\epsilon}}\right| d \mathcal{H}^{n-1},
\end{aligned}
$$

where $J G_{\mid \text {Tan } \partial \widetilde{\Omega}_{\epsilon}}$ denotes the Jacobian matrix of $G$ restricted to the tangent plane Tan $\partial \widetilde{\Omega}_{\epsilon}$ to $\partial \widetilde{\Omega}_{\epsilon}$. If $\tilde{g}:=g \circ G$ and $\widetilde{X}_{k}:=d G^{-1}\left[X_{k}\right]$, then

$$
\sum_{k=1}^{m} g_{k} X_{k}=d G\left[\sum_{k=1}^{m} \tilde{g}_{k} \tilde{X}_{k}\right]=d G\left[Y+\left\langle\sum_{k=1}^{m} \tilde{g}_{k} \tilde{X}_{k}, \boldsymbol{n}_{\widetilde{\Omega}_{\epsilon}}\right\rangle \boldsymbol{n}_{\widetilde{\Omega}_{\epsilon}}\right] \quad \text { on } \partial \widetilde{\Omega}_{\epsilon}
$$

for a suitable $Y \in$ Tan $\partial \widetilde{\Omega}_{\epsilon}=\boldsymbol{n}_{\widetilde{\Omega}_{\epsilon}}^{\perp}, \boldsymbol{n}_{\widetilde{\Omega}_{\epsilon}}$ being the Euclidean inner normal to $\partial \widetilde{\Omega}_{\epsilon}$. In particular $\left\langle\boldsymbol{n}_{\Omega_{\epsilon}} \circ G, d G[Y]\right\rangle=0$ (because $d G[Y] \in$ Tan $\partial \Omega_{\epsilon}$ ) and so

$$
\begin{aligned}
\left\langle\boldsymbol{n}_{\Omega_{\epsilon}} \circ G,\left(\sum_{k=1}^{m} g_{k} X_{k}\right) \circ G\right\rangle & =\left\langle\boldsymbol{n}_{\Omega_{\epsilon}} \circ G, d G[Y]+d G\left[\left\langle\sum_{k=1}^{m} \tilde{g}_{k} \widetilde{X}_{k}, \boldsymbol{n}_{\widetilde{\Omega}_{\epsilon}}\right\rangle \boldsymbol{n}_{\widetilde{\Omega}_{\epsilon}}\right]\right\rangle \\
& =\left\langle\sum_{k=1}^{m} \tilde{g}_{k} \widetilde{X}_{k}, \boldsymbol{n}_{\widetilde{\Omega}_{\epsilon}}\right\rangle\left\langle\boldsymbol{n}_{\Omega_{\epsilon}} \circ G, d G\left[\boldsymbol{n}_{\widetilde{\Omega}_{\epsilon}}\right]\right\rangle \quad \text { on } \partial \widetilde{\Omega}_{\epsilon} .
\end{aligned}
$$

From (4.14) one obtains

$$
\begin{aligned}
& \int_{\partial \Omega_{\epsilon}}\left\langle\boldsymbol{n}_{\Omega_{\epsilon}}, \sum_{k=1}^{m} g_{k} X_{k}\right\rangle d \mathcal{H}^{n-1} \\
= & \int_{\partial \widetilde{\Omega}_{\epsilon}}\left\langle\sum_{k=1}^{m} \tilde{g}_{k} \widetilde{X}_{k}, \boldsymbol{n}_{\widetilde{\Omega}_{\epsilon}}\right\rangle\left\langle\boldsymbol{n}_{\Omega_{\epsilon}} \circ G, d G\left[\boldsymbol{n}_{\widetilde{\Omega}_{\epsilon}}\right]\right\rangle \mid \operatorname{det} J G_{\mid \text {Tan } \partial \widetilde{\Omega}_{\epsilon} \mid d \mathcal{H}^{n-1}} \\
= & \int_{\partial \widetilde{\Omega}_{\epsilon}}\left\langle\sum_{k=1}^{m} \tilde{g}_{k} \tilde{X}_{k}, \boldsymbol{n}_{\widetilde{\Omega}_{\epsilon}}\right\rangle|\operatorname{det} J G| d \mathcal{H}^{n-1}
\end{aligned}
$$


where we have also used the fact that $\left\langle\boldsymbol{n}_{\Omega_{\epsilon}} \circ G, d G\left[\boldsymbol{n}_{\widetilde{\Omega}_{\epsilon}}\right]\right\rangle>0$, this inequality holding because $\boldsymbol{n}_{\widetilde{\Omega}_{\epsilon}}$ points inward $\widetilde{\Omega}_{\epsilon}$ and thus $d G\left[\boldsymbol{n}_{\widetilde{\Omega}_{\epsilon}}\right]$ points inward $\Omega_{\epsilon}$.

Let $\Phi_{\epsilon}: I \rightarrow \partial \widetilde{\Omega}_{\epsilon}$ be the parametrization of $\partial \widetilde{\Omega}_{\epsilon}$ defined by $\Phi_{\epsilon}(y):=$ $\left(y, \phi_{\epsilon}(y)\right)$; clearly, $\Phi_{\epsilon} \rightarrow \Phi$ uniformly on $I$. By (4.12) we have

$$
\boldsymbol{n}_{\widetilde{\Omega}_{\epsilon}} \circ \Phi_{\epsilon}=\frac{\nabla \tilde{f}}{|\nabla \tilde{f}|} \circ \Phi_{\epsilon}=\frac{\left(-\nabla \phi_{\epsilon}, 1\right)}{\sqrt{1+\left|\nabla \phi_{\epsilon}\right|^{2}}} \quad \text { on } I
$$

whence

$$
\frac{\partial_{s_{n}} \tilde{f}}{|\nabla \tilde{f}|} \circ \Phi_{\epsilon}=\frac{1}{\sqrt{1+\left|\nabla \phi_{\epsilon}\right|^{2}}} \text {, i.e., } \frac{\sqrt{1+\left|\nabla \phi_{\epsilon}\right|^{2}}}{|\nabla \tilde{f}| \circ \Phi_{\epsilon}}=\frac{1}{\left(\widetilde{X}_{j} \tilde{f}\right) \circ \Phi_{\epsilon}} \quad \text { on } I \text {. }
$$

From (4.15), (4.16), (4.17) and the area formula we obtain

$$
\begin{aligned}
& \int_{\partial \Omega_{\epsilon}}\left\langle\boldsymbol{n}_{\Omega_{\epsilon}}, \sum_{k=1}^{m} g_{k} X_{k}\right\rangle d \mathcal{H}^{n-1} \\
= & \int_{I}\left(\sum_{k=1}^{m} \tilde{g}_{k}\left\langle\widetilde{X}_{k}, \frac{\nabla \tilde{f}}{|\nabla \tilde{f}|}\right\rangle|\operatorname{det} J G|\right) \circ \Phi_{\epsilon} \sqrt{1+\left|\nabla \phi_{\epsilon}\right|^{2}} d \mathcal{L}^{n-1} \\
= & \int_{I}\left(\sum_{k=1}^{m} \tilde{g}_{k}|\operatorname{det} J G| \frac{\tilde{X}_{k} \tilde{f}}{\widetilde{X}_{j} \tilde{f}}\right) \circ \Phi_{\epsilon} d \mathcal{L}^{n-1} .
\end{aligned}
$$

and, recalling (4.13), we end up with

$$
-\int_{\Omega} \operatorname{div}_{X} g d \mathcal{L}^{n}=\lim _{\epsilon \rightarrow 0} \int_{I}\left\langle\tilde{g} \circ \Phi_{\epsilon},\left(|\operatorname{det} J G| \frac{\tilde{X} \tilde{f}}{\widetilde{X}_{j} \tilde{f}}\right) \circ \Phi_{\epsilon}\right\rangle d \mathcal{L}^{n-1},
$$

The functions

$$
\rho_{\epsilon}:=\left(|\operatorname{det} J G| \frac{\tilde{X} \tilde{f}}{\widetilde{X}_{j} \tilde{f}}\right) \circ \Phi_{\epsilon}
$$

are uniformly bounded in $L^{\infty}\left(I, \mathbb{R}^{m}\right)$ and, up to subsequences, we have $\rho_{\epsilon} \stackrel{*}{\rightarrow} \rho$ in $L^{\infty}\left(I, \mathbb{R}^{m}\right)=L^{1}\left(I, \mathbb{R}^{m}\right)^{*}$. Moreover $\tilde{g} \circ \Phi_{\epsilon} \rightarrow \tilde{g} \circ \Phi$ uniformly on $I$ and from (4.18) we get

$$
\begin{aligned}
\int_{\mathcal{Q}}\left\langle\nu_{\Omega}, g\right\rangle d|\partial \Omega|_{X} & =-\int_{\Omega} \operatorname{div}_{X} g d \mathcal{L}^{n} \\
& =\int_{I}\langle\tilde{g} \circ \Phi, \rho\rangle d \mathcal{L}^{n-1}=\int_{I}\langle g \circ G \circ \Phi, \rho\rangle d \mathcal{L}^{n-1}
\end{aligned}
$$

for any $g \in C_{c}^{1}\left(\mathcal{Q}, \mathbb{R}^{m}\right)$. Equality (4.10) follows and the proof is concluded. 
We warn the reader that the notation of Proposition 4.5 will be extensively utilized throughout the paper. We can now prove one of our main results.

Proof of Theorem 1.2. By Lemma 4.3, for any $x \in \partial \Omega$ there exist a neighbourhood $U \subset \mathbb{R}^{n}$, a Lipschitz function $f: U \rightarrow \mathbb{R}$ and $j \in\{1, \ldots, m\}$ such that $\Omega \cap U=$ $\{f>0\}$ or $\Omega \cap U=\{f<0\}$ and

$$
f \in C^{\infty}(U \backslash \partial \Omega), \quad \inf _{U \backslash \partial \Omega} X_{j} f>0 .
$$

If $\Omega \cap U=\{f>0\}$, then Proposition 4.5 (applied to $S:=\partial \Omega \cap U$ ) provides a neighbourhood $\mathcal{Q}$ of $x$ such that $|\partial \Omega|_{X}(\mathcal{Q})<+\infty$. If $\Omega \cap U=\{f<0\}$, then Proposition 4.5 provides a neighbourhood $\mathcal{Q}$ of $x$ such that $\left|\partial\left(\mathbb{R}^{n} \backslash \bar{\Omega}\right)\right|_{X}(\mathcal{Q})<$ $+\infty$. Recalling Corollary 4.2 we have $\mathcal{L}^{n}(\partial \Omega)=0$ and thus

$$
|\partial \Omega|_{X}(\mathcal{Q})=\left|\partial\left(\mathbb{R}^{n} \backslash \Omega\right)\right|_{X}(\mathcal{Q})=\left|\partial\left(\mathbb{R}^{n} \backslash \bar{\Omega}\right)\right|_{X}(\mathcal{Q})<\infty .
$$

We have proved that for any $x \in \partial \Omega$ there exists a neighbourhood $\mathcal{Q}$ such that $|\partial \Omega|_{X}(\mathcal{Q})<+\infty$ and this concludes the proof.

An easy consequence of Theorem 1.2 is the fact that intrinsic Lipschitz subgraphs in Carnot groups have locally finite $X$-perimeter. We use the same notation of Section 3.

Corollary 4.6. Let $\omega \subset \mathbb{W}$ be a bounded open set and $\phi: \omega \rightarrow \mathbb{R}$ an intrinsic Lipschitz function. Let $E_{\phi}:=\left\{y \cdot s e_{1}: y \in \omega, s<\phi(y)\right\}$ be the intrinsic subgraph of $\phi$. Then $\left|\partial E_{\phi}\right|_{X}(\omega \cdot \mathbb{R})<\infty$.

Proof. By Proposition 3.4 there exists an intrinsic Lipschitz function $\bar{\phi}: \mathbb{W} \rightarrow \mathbb{R}$ such that $\bar{\phi}_{\mid \omega}=\phi$. Since $\bar{\phi}$ is continuous, $\phi$ is bounded on $\omega$, say $a<\phi<b$ for suitable real numbers $a, b$. Thus we have

$$
\left|\partial E_{\phi}\right|_{X}(\omega \cdot \mathbb{R})=\left|\partial E_{\bar{\phi}}\right|_{X}(\omega \cdot(a, b))<\infty
$$

because $E_{\bar{\phi}}:=\left\{y \cdot s e_{1}: y \in \mathbb{W}, s<\bar{\phi}(y)\right\}$ is an $X$-Lipschitz domain (see Remark 3.5).

Some remarks are in order.

Remark 4.7. Using the same notation of Proposition 4.5 and its proof, we explicitly notice that, for sufficiently small $\epsilon>0$, the vector functions $\rho_{\epsilon}$ belong to $L^{\infty}(I) \cap C^{\infty}(I)$ and

$$
v_{\Omega_{\epsilon}}\left|\partial \Omega_{\epsilon}\right|_{X}\left\llcorner\mathcal{Q}=\left(G \circ \Phi_{\epsilon}\right)_{\#}\left(\rho_{\epsilon} \mathcal{L}^{n-1}\llcorner I) .\right.\right.
$$

When $\partial \Omega$ is also $X$-regular, it follows from Lemma 4.4 that the function $f$ can be chosen in the class $C^{\infty}(U \backslash S) \cap C_{X}^{1}(U)$. This implies that $\tilde{X} \tilde{f}$ is continuous, whence $\rho_{\epsilon}$ converges locally uniformly on $I$ to the continuous function

$$
\rho=\left(\frac{\tilde{X} \tilde{f}}{\widetilde{X}_{j} \tilde{f}}|\operatorname{det} J G|\right) \circ \Phi .
$$


In particular, from (4.10) one obtains that the measure theoretic normal is continuous and coincides with the horizontal normal to $\partial \Omega$,

$$
v_{\Omega}=\frac{X f}{|X f|} \quad|\partial \Omega|_{X} \text {-a.e. on } \partial \Omega \text {. }
$$

Remark 4.8. Using the same notation of Proposition 4.5, it is easily seen that $c \leqslant$ $\left|\rho_{\epsilon}\right| \leqslant C$ for some positive constants $c, C$ independent of $\epsilon$. This implies that $c \leqslant|\rho| \leqslant C$ and, in particular, that a function $w$ belongs to $L^{1}\left(\partial \Omega \cap \mathcal{Q},|\partial \Omega|_{X}\right)$ if and only if $w \circ G \circ \Phi$ belongs to $L^{1}\left(I, \mathcal{L}^{n-1}\right)$. Moreover, a sequence $\left(w_{k}\right)_{k}$ converges to $w$ in $L^{1}\left(\partial \Omega \cap \mathcal{Q},|\partial \Omega|_{X}\right)$ if and only if $w_{k} \circ G \circ \Phi \rightarrow w \circ G \circ \Phi$ in $L^{1}\left(I, \mathcal{L}^{n-1}\right)$.

Remark 4.9. It is interesting to interpret Propositions 4.1 and 4.5 in the setting of Carnot groups. Assume $S \subset \mathbb{G}$ is an $X$-Lipschitz surface and $x \in S$. Without loss of generality, we may assume that $S$ is the intrinsic Lipschitz graph of $\phi: \omega \rightarrow \mathbb{R}$, for some $\omega \subset \mathbb{W}$ open, and that $x=0$, whence $\phi(0)=0$. It is easily seen that the diffeomorphism $G$ provided by Proposition 4.1 takes the form

$$
\begin{aligned}
G: \omega \times \mathbb{R} & \rightarrow \mathbb{G} \\
(y, h) & \mapsto y \cdot h e_{1} ;
\end{aligned}
$$

in other words, one can take $I=\omega \subset \mathbb{W} \equiv \mathbb{R}^{n-1}$ and the implicit function provided by Proposition 4.1 is exactly $\phi$. Moreover, Proposition 4.5 implies that

$$
\left|\partial E_{\phi}\right|_{X}=\Phi_{\#}\left(\rho \mathcal{L}^{n-1}\llcorner\omega)\right.
$$

for a suitable $\rho \in L^{\infty}(\omega)$.

Assume now that $S$ is also $X$-regular, i.e., $S=\{f=0\}$ for some $f$ of class $C_{X}^{1}$ with $X_{1} f>0$. It turns out that det $J G=1$ and by Remark 4.7 we recover

$$
\left|\partial E_{\phi}\right|_{X}=\frac{|X f|}{X_{1} f} \Phi_{\#}\left(\mathcal{L}^{n-1}\llcorner\omega),\right.
$$

which is one of the main results of [35].

\subsection{Ahlfors regularity of the $X$-perimeter for $X$-Lipschitz domains in equire- gular CC spaces}

Let $\mathbb{R}^{n}$ be a CC space whose structure is induced by a family $X=\left(X_{1}, \ldots, X_{m}\right)$. Given $x \in \mathbb{R}^{n}$ and $i \in \mathbb{N}$ we define $\mathfrak{L}_{i}(x)$ as the linear span (at $x$ ) of all the commutators of $X_{1}, \ldots, X_{m}$ up to order $i$. We say that $\left(\mathbb{R}^{n}, X\right)$ is an equiregular CC space if $n_{i}:=\operatorname{dim} \mathfrak{L}_{i}(x)$ does not depend on $x$ and $n_{\kappa}=n$ for some $\kappa$; we assume $\kappa$ to be minimal and call it step of the CC space. In particular, rank $\mathfrak{L}_{\kappa}(x)=$ $n$ for any $x$ and the Chow-Hörmander condition is satisfied together with (2.1). Let us set also $n_{0}:=0$ and $m_{i}:=n_{i}-n_{i-1}$, so that $m_{1}=m$; it is well-known (see [48]) that the Hausdorff dimension of $\left(\mathbb{R}^{n}, d\right)$ is $Q:=\sum_{i=1}^{\kappa} i m_{i}$. 
It is worth mentioning that the $\mathrm{CC}$ distance is locally $\frac{1}{\kappa}$-Hölder continuous with respect to the Euclidean distance in $\mathbb{R}^{n}$ (see [52]); namely, for any $K \Subset \mathbb{R}^{n}$ there exists $M=M(K)>0$ such that

$$
d\left(x, x^{\prime}\right) \leqslant M\left|x-x^{\prime}\right|^{1 / \kappa} \quad \text { for any } x, x^{\prime} \in K .
$$

This fact provides the key tool in the proof of the following result, where we show that $X$-Lipschitz surfaces in equiregular spaces are locally (and up to a diffeomorphism of the ambient space) graphs of $\frac{1}{\kappa}$-Hölder continuous functions.

Proposition 4.10. Under the same assumptions of Proposition 4.1. If $\left(\mathbb{R}^{n}, X\right)$ is an equiregular space, then $\mathcal{Q}, I, a, G, \phi$ in Proposition 4.1 can be chosen so that $\phi$ is $\frac{1}{\kappa}$-Hölder continuous on $I$.

Proof. Let

$$
\mathcal{Q}, I, a, G, \phi, \tilde{f}, l
$$

be defined as in Proposition 4.1 and its proof. Up to a localization argument we may assume that

$$
d\left(x, x^{\prime}\right) \leqslant M\left|x-x^{\prime}\right|^{1 / \kappa} \quad \text { for any } x, x^{\prime} \in \mathcal{Q}
$$

for some positive $M$. Let $y, y^{\prime} \in I$ be fixed; for $\tau \in(0, a / 2)$ we have $^{2}$

$$
\tilde{f}(y, \phi(y)+\tau)=\tilde{f}\left(\exp \left(\tau \partial_{s_{n}}\right)(y, \phi(y))\right) \geqslant \tilde{f}(y, \phi(y))+\tau l=\tau l,
$$

where we have used the inequality $\partial_{s_{n}} \tilde{f} \geqslant l$ and Lemma 2.2. If $L>0$ is the Lipschitz constant of $f$ we get

$$
\begin{aligned}
\tilde{f}\left(y^{\prime}, \phi(y)+\tau\right) & =f\left(G\left(y^{\prime}, \phi(y)+\tau\right)\right) \\
& \geqslant f(G(y, \phi(y)+\tau))-L d\left(G\left(y^{\prime}, \phi(y)+\tau\right), G(y, \phi(y)+\tau)\right) \\
& \geqslant \tilde{f}(y, \phi(y)+\tau)-L M\left|G\left(y^{\prime}, \phi(y)+\tau\right)-G(y, \phi(y)+\tau)\right|^{1 / \kappa} \\
& \geqslant \tau l-L M\|\nabla G\|_{\infty}^{1 / \kappa}\left|y-y^{\prime}\right|^{1 / \kappa} .
\end{aligned}
$$

If $\left|y-y^{\prime}\right|$ is small enough, in such a way that the number

$$
\bar{\tau}:=\frac{L M\|\nabla G\|_{\infty}^{1 / \kappa}}{l}\left|y-y^{\prime}\right|^{1 / \kappa}
$$

is smaller than $a / 2$, we get $\tilde{f}\left(y^{\prime}, \phi(y)+\bar{\tau}\right) \geqslant 0$, i.e.,

$$
\phi\left(y^{\prime}\right) \leqslant \phi(y)+\bar{\tau}=\phi(y)+\frac{L M\|\nabla G\|_{\infty}^{1 / \kappa}}{l}\left|y-y^{\prime}\right|^{1 / \kappa} .
$$

2 The assumption $\tau<a / 2$ gives $|\phi(y)+\tau|<a$ for any $y \in I$, so that the following quantities are well defined. 
A similar argument gives the other inequality

$$
\phi\left(y^{\prime}\right) \geqslant \phi(y)-\bar{\tau}=\phi(y)-\frac{L M\|\nabla G\|_{\infty}^{1 / \kappa}}{l}\left|y-y^{\prime}\right|^{1 / \kappa}
$$

and the proof is accomplished.

We will need in the sequel a variant of the classical Ball-Box Theorem by Nagel, Stein and Wainger [52] proved by D. Morbidelli in [51]. Before stating it, let us introduce some preliminary notation.

Denote by $Y_{1}, \ldots, Y_{q}$ a fixed enumeration of all the commutators of the vectors $X_{1}, \ldots, X_{m}$ of length at most $\kappa$; let $d\left(Y_{k}\right) \in\{1, \ldots, \kappa\}$ denote the length of the commutator $Y_{k}$. We will need the notion of "almost exponential" map, exp*, introduced in [51], to which we refer for the precise definition. Here we only recall that

- for any $z \in \mathbb{R}^{n}$ there exists $t_{0}=t_{0}(z)>0$ such that $\left(-t_{0}, t_{0}\right) \ni t \mapsto$ $\exp ^{*}\left(t Y_{k}\right)(z) \in \mathbb{R}^{n}$ defines a piecewise smooth horizontal curve. Moreover, $t_{0}=t_{0}(z)$ is continuous with respect to $z$

- the map $z \mapsto \exp ^{*}\left(t Y_{k}\right)(z)$ is smooth (when defined);

- if $d\left(Y_{k}\right)=1$, then $\exp ^{*}\left(t Y_{k}\right)(z)=\exp \left(t Y_{k}\right)(z)$.

Given a multi-index $\mathcal{I}=\left(i_{1}, \ldots, i_{n}\right) \in\{1, \ldots, q\}^{n}$, define

$$
\begin{aligned}
& d(\mathcal{I}):=d\left(Y_{i_{1}}\right)+\cdots+d\left(Y_{i_{n}}\right) \\
& \lambda_{\mathcal{I}}(z):=\operatorname{det}\left[Y_{i_{1}}(z), \ldots, Y_{i_{n}}(z)\right] \\
& \|h\|_{\mathcal{I}}:=\max _{k=1, \ldots, n}\left|h_{k}\right|^{1 / d\left(Y_{i_{k}}\right)}, \quad h \in \mathbb{R}^{n}
\end{aligned}
$$

and the map

$$
E_{\mathcal{I}}(z, h):=\exp ^{*}\left(h_{1} Y_{i_{1}}\right) \exp ^{*}\left(h_{2} Y_{i_{2}}\right) \ldots \exp ^{*}\left(h_{n} Y_{i_{n}}\right)(z) .
$$

We denote by $\mathcal{B}(z, r)$ the set $\left\{E_{\mathcal{I}}(z, h):\|h\|_{\mathcal{I}}<r\right\}$; the sets $\mathcal{B}(z, r)$ play the role of pseudo-balls according to the following result (see [51, Theorem 3.1 and Lemma 3.3]).

Theorem 4.11. Let $K \subset \mathbb{R}^{n}$ be a compact set; then, there exist positive numbers $\hat{r}, \alpha, \beta, \beta<\alpha<1$, such that the following holds. For any $z \in K, r \in(0, \hat{r})$ and $\mathcal{I}$ such that

$$
\left|\lambda_{\mathcal{I}}(z)\right| r^{d(\mathcal{I})}>\frac{1}{2} \max _{\mathcal{J}}|\lambda \mathcal{J}(z)| r^{d(\mathcal{J})},
$$

one has

(1) if $\|h\|_{\mathcal{I}} \leqslant \alpha r$, then $E_{\mathcal{I}}(z, \cdot)$ is differentiable at $h$ and

$$
\frac{1}{4}\left|\lambda_{\mathcal{I}}(z)\right| \leqslant\left|J_{h} E_{\mathcal{I}}(z, h)\right| \leqslant 4\left|\lambda_{\mathcal{I}}(z)\right|,
$$

where $J_{h} E_{\mathcal{I}}(z, h)$ denotes the Jacobian determinant of $E_{\mathcal{I}}(z, \cdot)$; 
(2) $B(z, \beta r) \subset \mathcal{B}(z, \alpha r) \subset B(z, r)$;

(3) $E_{\mathcal{I}}(z, \cdot)$ is one-to-one on $\left\{\|h\|_{\mathcal{I}}<\alpha r\right\}$.

Remark 4.12. The precise statement of Theorem 4.11 (2) given in [51] is

$$
B_{\rho}(z, \beta r) \subset \mathcal{B}(z, \alpha r) \subset B(z, r),
$$

where $\rho$ is a suitable distance defined in [51, p. 217]. Such a distance is equivalent to the CC distance $d$ and $\rho \leqslant d$, thus $B(z, \beta r) \subset B_{\rho}(z, \beta r)$ and our statement follows.

Theorem 4.11 can be refined in equiregular CC spaces. In this case, in fact, if $z \in \mathbb{R}^{n}$ is fixed and $r$ is small enough, inequality (4.20) may hold only if $\left|\lambda_{\mathcal{I}}(z)\right| \neq$ 0 and $d(\mathcal{I})$ is minimal, i.e., $d(\mathcal{I})=Q$. Using also the continuity of the $\lambda_{\mathcal{J}}$ 's, it is not difficult to show that

for any $x \in \mathbb{R}^{n}$ there exist a bounded open neighbourhood $U$ of $x$, a multi-index $\mathcal{I}$ and $r_{0}>0$ such that $d(\mathcal{I})=Q$ and (4.20) holds for any $z \in U$ and $r \in\left(0, r_{0}\right)$.

Possibly restricting this number $r_{0}$, we may assume that it is smaller than the number $\hat{r}$ provided by Theorem 4.11 (applied to $K:=\bar{U}$ ).

We can now prove the Ahlfors regularity of the $X$-perimeter measure for $X$ Lipschitz domains in equiregular CC spaces.

Proof of Theorem 1.3. Let $x \in \partial \Omega$; due to the compactness of $\partial \Omega$, it will be enough to prove that there exists a suitable neighbourhood $\mathcal{A}$ of $x$ such that (1.1) holds for any $z \in \partial \Omega \cap \mathcal{A}$. Let $\mathcal{I}=\left(i_{1}, \ldots, i_{n}\right), U$ and $r_{0}$ be as in (4.21) and set $Z_{k}:=Y_{i_{k}}$; up to reordering $\mathcal{I}$ and restricting $U$ we may assume that

$$
\mathfrak{L}_{i}(z)=\operatorname{span}\left\{Z_{1}(z), \ldots, Z_{n_{i}}(z)\right\} \text { for any } z \in U \text { and } i=1, \ldots, \kappa .
$$

In particular, $\left\{Z_{1}, \ldots, Z_{m}\right\}=\left\{X_{1}, \ldots, X_{m}\right\}$. Possibly restricting $U$, by Lemma 4.3 we may assume that there exist a Lipschitz function $f: U \rightarrow \mathbb{R}$ and $j \in\{1, \ldots, m\}$ such that

- $\Omega \cap U=\{f>0\}$ or $\Omega \cap U=\{f<0\}$;

- $f \in C^{\infty}(U \backslash \partial \Omega)$ and $l:=\inf _{U \backslash \partial \Omega} X_{j} f>0$.

Since $|\partial \Omega|_{X}=\left|\partial\left(\mathbb{R}^{n} \backslash \Omega\right)\right|_{X}=\left|\partial\left(\mathbb{R}^{n} \backslash \bar{\Omega}\right)\right|_{X}$ (because $\mathcal{L}^{n}(\partial \Omega)=0$ ), we may assume without loss of generality that $\Omega \cap U=\{f>0\}$. Let $\mathcal{Q} \subset U$ be the open neighbourhood of $x$ given by Proposition 4.5 (applied to $S:=\partial \Omega \cap U$ ). We also define $\widetilde{Z}_{k}:=d G^{-1}\left[Z_{k}\right]$; since it is not restrictive to assume $Z_{1}=X_{j}$, we have $\widetilde{Z}_{1}=\partial_{s_{n}}$. Let

$$
I, a, G, \phi, \Phi, \tilde{f}, \widetilde{\Omega}, \rho
$$

be defined as in Proposition 4.5 and its proof. We also fix an open set $I^{\prime} \Subset I$ and, up to restricting $r_{0}$, we may assume that $B\left(z, r_{0}\right) \subset \mathcal{Q}$ for any $z \in \mathcal{A}:=$ 
$G\left(I^{\prime} \times(-a / 2, a / 2)\right) \Subset \mathcal{Q}$. Let $L$ be the Lipschitz constant of $f$ and $\alpha, \beta$ be given by Theorem 4.11 (applied to $K:=\bar{U}$ ). Set

$$
\bar{r}:=\min \left\{\beta r_{0}, \frac{r_{0}}{\alpha\left(1+\frac{l}{L}\right)}\right\}<r_{0} .
$$

We claim that (1.1) holds for any $z \in \partial \Omega \cap \mathcal{A}$ and any $r<\bar{r}$; this would conclude the proof.

We begin by proving the second inequality in (1.1) for such $z, r$. We set $\pi_{I}$ : $I \times(-a, a) \rightarrow I$ to be the canonical projection and

$$
\widetilde{B}\left(G^{-1}(z), r\right):=G^{-1}(B(z, r)) \text { and } \tilde{\mathcal{B}}\left(G^{-1}(z), r\right):=G^{-1}(\mathcal{B}(z, r)) .
$$

Since $r / \beta<r_{0} \leqslant \hat{r}$, by Theorem 4.11 (2) we have

$$
B(z, r) \subset \mathcal{B}\left(z, \frac{\alpha}{\beta} r\right) \subset B\left(z, r_{0}\right) \subset \mathcal{Q}
$$

whence, on considering the images of these sets under the map $\pi_{I} \circ G^{-1}$,

$$
\pi_{I}\left(\widetilde{B}\left(G^{-1}(z), r\right)\right) \subset \pi_{I}\left(\widetilde{\mathcal{B}}\left(G^{-1}(z), \frac{\alpha}{\beta} r\right)\right)=\pi_{I}\left(G^{-1}\left(\left\{E_{\mathcal{I}}(z, h):\|h\|_{\mathcal{I}}<\frac{\alpha}{\beta} r\right\}\right)\right) .
$$

Writing $h=\left(h_{1}, h^{\prime}\right) \in \mathbb{R} \times \mathbb{R}^{n-1}$, we can decompose the map $E_{\mathcal{I}}$ as $E_{\mathcal{I}}(z, h)=$ $\exp \left(h_{1} Z_{1}\right) E_{\mathcal{I}}^{\prime}\left(z, h^{\prime}\right)$ where

$$
E_{\mathcal{I}}^{\prime}\left(z, h^{\prime}\right)=\exp ^{*}\left(h_{2} Z_{2}\right) \ldots \exp ^{*}\left(h_{n} Z_{n}\right)(z) .
$$

Since $d G^{-1}\left[Z_{1}\right]=\partial_{S_{n}}$ we obtain

$$
\pi_{I}\left(G^{-1}\left(E_{\mathcal{I}}(z, h)\right)\right)=\pi_{I}\left(\exp \left(h_{1} \partial_{s_{n}}\right) G^{-1}\left(E_{\mathcal{I}}^{\prime}\left(z, h^{\prime}\right)\right)\right)=\pi_{I}\left(G^{-1}\left(E_{\mathcal{I}}^{\prime}\left(z, h^{\prime}\right)\right)\right)
$$

and from (4.22) one gets

$$
\begin{aligned}
\pi_{I}\left(\widetilde{B}\left(G^{-1}(z), r\right)\right) & \subset \pi_{I}\left(G^{-1}\left(\left\{E_{\mathcal{I}}^{\prime}\left(z, h^{\prime}\right):\left\|h^{\prime}\right\|_{\mathcal{I}}<\frac{\alpha}{\beta} r\right\}\right)\right) \\
& =P_{z}\left(\left\{h^{\prime} \in \mathbb{R}^{n-1}:\left\|h^{\prime}\right\|_{\mathcal{I}}<\frac{\alpha}{\beta} r\right\}\right)
\end{aligned}
$$

where we have set $P_{z}:=\left(\pi_{I} \circ G^{-1} \circ E_{\mathcal{I}}^{\prime}\right)(z, \cdot): \mathbb{R}^{n-1} \rightarrow I$ and $\left\|h^{\prime}\right\|_{\mathcal{I}}:=\left\|\left(0, h^{\prime}\right)\right\|_{\mathcal{I}}$ for $h^{\prime} \in \mathbb{R}^{n-1}$. The map $E_{\mathcal{I}}(z, \cdot)$ is differentiable on $\left\{\|h\|_{\mathcal{I}}<\alpha r\right\}$; moreover, $E_{\mathcal{I}}(\cdot, h)$ is smooth. Therefore, also

$$
h \longmapsto G^{-1} \circ E_{\mathcal{I}}(z, h)=\exp \left(h_{1} \partial_{s_{n}}\right) G^{-1}\left(E_{\mathcal{I}}^{\prime}\left(z, h^{\prime}\right)\right)
$$

is differentiable on $\left\{\|h\|_{\mathcal{I}}<\alpha r\right\}$ and smooth with respect to $z$. From Theorem 4.11 (1) we obtain

$\frac{1}{4}\left|\operatorname{det} G^{-1}(z)\right|\left|\lambda_{\mathcal{I}}(z)\right| \leqslant\left|J_{h}\left(G^{-1} \circ E_{\mathcal{I}}\right)(z, h)\right| \leqslant 4\left|\operatorname{det} G^{-1}(z)\right|\left|\lambda_{\mathcal{I}}(z)\right|$ if $\|h\|_{\mathcal{I}}<\alpha r$ 
and, in particular, there exist positive constants $C_{1}, C_{2}$ such that

$$
C_{1} \leqslant\left|J_{h}\left(G^{-1} \circ E_{\mathcal{I}}\right)(z, h)\right| \leqslant C_{2} \text { for any } h \in \mathbb{R}^{n} \text { with }\|h\|_{\mathcal{I}}<\alpha r .
$$

Notice that $\frac{\partial}{\partial h_{1}}\left(G^{-1} \circ E_{\mathcal{I}}\right)=d G^{-1}\left[Z_{1}\right]=\partial_{s_{n}}$, thus the first row of $\nabla_{h}\left(G^{-1} \circ\right.$ $\left.E_{\mathcal{I}}\right)(z, h)$ (namely, the one corresponding to derivatives with respect to $h_{1}$ ) is $(0, \ldots, 0,1)$. It follows that

$$
C_{1} \leqslant\left|\operatorname{det} M\left(z, h^{\prime}\right)\right| \leqslant C_{2} \text { for any } h^{\prime} \in \mathbb{R}^{n-1} \text { with }\left\|h^{\prime}\right\|_{\mathcal{I}}<\alpha r
$$

where $M\left(z, h^{\prime}\right)$ is the $(n-1) \times(n-1)$ minor of the Jacobian matrix $\nabla_{h}\left(G^{-1}\right.$ 。 $\left.E_{\mathcal{I}}\right)\left(z,\left(0, h^{\prime}\right)\right)$ obtained by erasing the first row and the last column (the one corresponding to the $n$-th coordinate in $I \times(-a, a))$. It is easily seen that

$M\left(z, h^{\prime}\right)=\nabla_{h^{\prime}}\left(\pi_{I} \circ G^{-1} \circ E_{\mathcal{I}}^{\prime}\right)\left(z, h^{\prime}\right)=\nabla_{h^{\prime}} P_{z}\left(h^{\prime}\right)$ for any $h^{\prime} \in \mathbb{R}^{n-1}$ with $\left\|h^{\prime}\right\|_{\mathcal{I}}<\alpha r$ so that $C_{1} \leqslant J_{h^{\prime}} P_{z}\left(h^{\prime}\right) \leqslant C_{2}$. By the area formula

$$
\begin{aligned}
\mathcal{L}^{n-1}\left(\pi_{I}\left(\partial \widetilde{\Omega} \cap \widetilde{B}\left(G^{-1}(z), r\right)\right)\right) & \leqslant \mathcal{L}^{n-1}\left(\pi_{I}\left(\widetilde{B}\left(G^{-1}(z), r\right)\right)\right) \\
& \left.\leqslant \mathcal{L}^{n-1}\left(P_{z}\left(\left\{\left\|h^{\prime}\right\|_{\mathcal{I}}<\frac{\alpha}{\beta} r\right\}\right)\right) \quad \text { (by }(4.23)\right) \\
& \leqslant C_{2} \mathcal{L}^{n-1}\left(\left\{\left\|h^{\prime}\right\|_{\mathcal{I}}<\frac{\alpha}{\beta} r\right\}\right)=C_{3} r^{Q-1} .
\end{aligned}
$$

and from (4.11) we get

$$
|\partial \Omega|_{X}(B(z, r))=\int_{\pi_{I}\left(\partial \widetilde{\Omega} \cap \widetilde{B}\left(G^{-1}(z), r\right)\right)}|\rho| d \mathcal{L}^{n-1} \leqslant C_{4} r^{Q-1}
$$

as desired.

Let us prove the first inequality in (1.1) for $z \in \partial \Omega \cap \mathcal{A}$ and $r<\bar{r}$. It is enough to show that for

$$
C_{5}:=\min \left\{\alpha, \frac{\alpha^{2} l}{L}, \frac{r_{0}}{\bar{r}}, \frac{\alpha^{2} l \hat{r}}{2 L \bar{r}}\right\}
$$

it holds

$$
P_{z}\left(\left\{h^{\prime} \in \mathbb{R}^{n-1}:\left\|h^{\prime}\right\|_{\mathcal{I}}<C_{5} r\right\}\right) \subset \pi_{I}\left(\partial \widetilde{\Omega} \cap \widetilde{B}\left(G^{-1}(z), r\right)\right) .
$$

Indeed, from (4.25) and (4.24) it would follow that

$$
\begin{aligned}
\mathcal{L}^{n-1}\left(\pi_{I}\left(\partial \widetilde{\Omega} \cap \widetilde{B}\left(G^{-1}(z), r\right)\right)\right) & \geqslant \mathcal{L}^{n-1}\left(P_{z}\left(\left\{\left\|h^{\prime}\right\|_{\mathcal{I}}<C_{5} r\right\}\right)\right) \\
& \geqslant C_{1} \mathcal{L}^{n-1}\left(\left\{\left\|h^{\prime}\right\|_{\mathcal{I}}<C_{5} r\right\}\right)=C_{6} r{ }^{Q-1},
\end{aligned}
$$

where we have used area formula and the fact, which will be proved later, that $P_{z}$ is one-to-one on $\left\{\left\|h^{\prime}\right\|_{\mathcal{I}}<C_{5} r\right\}$. By Remark 4.8

$$
|\partial \Omega|_{X}(B(z, r)) \geqslant \int_{\pi_{I}\left(\partial \widetilde{\Omega} \cap \widetilde{B}\left(G^{-1}(z), r\right)\right)}|\rho| d \mathcal{L}^{n-1} \geqslant C_{7} r^{Q-1}
$$

as desired. 
To prove (4.25), it is sufficient to show the implication

$$
\begin{aligned}
& h^{\prime} \in \mathbb{R}^{n-1},\left\|h^{\prime}\right\|_{\mathcal{I}}<C_{5} r \\
\Rightarrow & \exists h_{1}=h_{1}\left(h^{\prime}\right) \in(-\alpha r, \alpha r) \text { such that } E_{\mathcal{I}}\left(z,\left(h_{1}, h^{\prime}\right)\right) \in \partial \Omega ;
\end{aligned}
$$

in fact, from (4.26) one would get the chain of implications

$$
\begin{aligned}
& h^{\prime} \in \mathbb{R}^{n-1},\left\|h^{\prime}\right\|_{\mathcal{I}}<C_{5} r \\
\Rightarrow & \left.\left.E_{\mathcal{I}}\left(z,\left(h_{1}, h^{\prime}\right)\right)\right) \in \partial \Omega \cap \mathcal{B}(z, \alpha r) \quad \text { (because }\left\|\left(h_{1}, h^{\prime}\right)\right\|_{\mathcal{I}}<\alpha r\right) \\
\Rightarrow & \left.E_{\mathcal{I}}\left(z,\left(h_{1}, h^{\prime}\right)\right)\right) \in \partial \Omega \cap B(z, r) \quad \text { (by Theorem 4.11(2)) } \\
\Rightarrow & G^{-1}\left(E_{\mathcal{I}}\left(z,\left(h_{1}, h^{\prime}\right)\right)\right) \in G^{-1}(\partial \Omega \cap B(z, r))=\partial \widetilde{\Omega} \cap \widetilde{B}\left(G^{-1}(z), r\right) \\
\Rightarrow & P_{z}\left(h^{\prime}\right)=\pi_{I}\left(G^{-1}\left(E_{\mathcal{I}}^{\prime}\left(z, h^{\prime}\right)\right)\right) \\
& =\pi_{I}\left(G^{-1}\left(E_{\mathcal{I}}\left(z,\left(h_{1}, h^{\prime}\right)\right)\right)\right) \in \pi_{I}\left(\partial \widetilde{\Omega} \cap \widetilde{B}\left(G^{-1}(z), r\right)\right)
\end{aligned}
$$

which is (4.25).

Let us prove (4.26). Fix $h^{\prime} \in \mathbb{R}^{n-1}$ with $\left\|h^{\prime}\right\|_{\mathcal{I}}<C_{5} r$. By Theorem 4.11 (2) (which can be applied because $C_{5} r<C_{5} \bar{r} \leqslant r_{0} \leqslant \hat{r}$ ) one gets $d\left(z, E_{\mathcal{I}}^{\prime}\left(z, h^{\prime}\right)\right)<$ $\frac{C_{5}}{\alpha} r \leqslant \alpha \frac{l}{L} r$, whence

$$
\left|f\left(E_{\mathcal{I}}^{\prime}\left(z, h^{\prime}\right)\right)\right| \leqslant|f(z)|+L d\left(z, E_{\mathcal{I}}^{\prime}\left(z, h^{\prime}\right)\right)<\alpha l r
$$

because $f(z)=0$. Since $X_{j} f=Z_{1} f \geqslant l$ on $\mathcal{Q}$ we deduce by Lemma 2.2

$$
\begin{aligned}
& f\left(E_{\mathcal{I}}\left(z,\left(\alpha r, h^{\prime}\right)\right)\right)=f\left(\exp \left(\alpha r Z_{1}\right) E_{\mathcal{I}}^{\prime}\left(z, h^{\prime}\right)\right) \geqslant f\left(E_{\mathcal{I}}^{\prime}\left(z, h^{\prime}\right)\right)+\alpha l r>0 \\
& f\left(E_{\mathcal{I}}\left(z,\left(-\alpha r, h^{\prime}\right)\right)\right)=f\left(\exp \left(-\alpha r Z_{1}\right) E_{\mathcal{I}}^{\prime}\left(z, h^{\prime}\right)\right) \leqslant f\left(E_{\mathcal{I}}^{\prime}\left(z, h^{\prime}\right)\right)-\alpha l r<0 .
\end{aligned}
$$

Notice that Lemma 2.2 can be applied because for any $t \in(-\alpha r, \alpha r)$

$$
d\left(z, E_{\mathcal{I}}\left(z,\left(t, h^{\prime}\right)\right)\right) \leqslant|t|+d\left(z, E_{\mathcal{I}}^{\prime}\left(z, h^{\prime}\right)\right) \leqslant \alpha r+\alpha \frac{l}{L} r<\bar{r} \alpha\left(1+\frac{l}{L}\right) \leqslant r_{0},
$$

i.e., $E_{\mathcal{I}}\left(z,\left(t, h^{\prime}\right)\right) \in B\left(z, r_{0}\right) \subset \mathcal{Q}$ for any such $t$.

By (4.27) and the continuity of $t \mapsto f\left(E_{\mathcal{I}}\left(z,\left(t, h^{\prime}\right)\right)\right)$, there exists $h_{1} \in$ $(-\alpha r, \alpha r)$ such that $f\left(E_{\mathcal{I}}\left(z,\left(h_{1}, h^{\prime}\right)\right)\right)=0$. This means that $E_{\mathcal{I}}\left(z,\left(h_{1}, h^{\prime}\right)\right) \in \partial \Omega$ and (4.26) follows.

It is only left to prove that $P_{z}$ is one-to-one on $\left\{\left\|h^{\prime}\right\|_{\mathcal{I}}<C_{5} r\right\}$. Assume not: then there exist $h^{\prime} \neq h^{\prime \prime}$ in $\mathbb{R}^{n-1}$ such that $\left\|h^{\prime}\right\|_{\mathcal{I}}<C_{5} r,\left\|h^{\prime \prime}\right\|_{\mathcal{I}}<C_{5} r$ and $P_{z}\left(h^{\prime}\right)=P_{z}\left(h^{\prime \prime}\right)$, i.e.,

$$
G^{-1}\left(E_{\mathcal{I}}^{\prime}\left(z, h^{\prime}\right)\right)=\left(y, t^{\prime}\right) \quad \text { and } \quad G^{-1}\left(E_{\mathcal{I}}^{\prime}\left(z, h^{\prime \prime}\right)\right)=\left(y, t^{\prime \prime}\right)
$$

for suitable $y \in I$ and $t^{\prime}, t^{\prime \prime} \in(-a, a)$. We have $t^{\prime} \neq t^{\prime \prime}$ because, due to Theorem 4.11 (3) and the inequality $C_{5} \leqslant \alpha, E_{\mathcal{I}}^{\prime}$ (as well as $G^{-1} \circ E_{\mathcal{I}}^{\prime}$ ) is one-to-one on 
$\left\{\left\|h^{\prime}\right\|_{\mathcal{I}}<C_{5} r\right\}$. Since $\left(y, t^{\prime}\right)=\exp \left(\left(t^{\prime}-t^{\prime \prime}\right) \partial_{S_{n}}\right)\left(y, t^{\prime \prime}\right)$ we have by Lemma 2.2 and Theorem 4.11 (2)

$$
\begin{aligned}
\left|t^{\prime}-t^{\prime \prime}\right| & \leqslant \frac{1}{l}\left|\tilde{f}\left(y, t^{\prime}\right)-\tilde{f}\left(y, t^{\prime \prime}\right)\right| \leqslant \frac{L}{l} d\left(E_{\mathcal{I}}^{\prime}\left(z, h^{\prime}\right), E_{\mathcal{I}}^{\prime}\left(z, h^{\prime \prime}\right)\right) \\
& \leqslant \frac{L}{l} d\left(E_{\mathcal{I}}^{\prime}\left(z, h^{\prime}\right), z\right)+\frac{L}{l} d\left(z, E_{\mathcal{I}}^{\prime}\left(z, h^{\prime \prime}\right)\right) \\
& \leqslant 2 \frac{L}{l} \frac{C_{5} r}{\alpha}<\alpha \hat{r} .
\end{aligned}
$$

Thus $\left\|\left(t^{\prime}-t^{\prime \prime}, h^{\prime \prime}\right)\right\|_{\mathcal{I}}<\alpha \hat{r}$ and

$$
E_{\mathcal{I}}^{\prime}\left(z, h^{\prime}\right)=\exp \left(\left(t^{\prime}-t^{\prime \prime}\right) X_{j}\right)\left(E_{\mathcal{I}}^{\prime}\left(z, h^{\prime \prime}\right)\right)=E_{\mathcal{I}}\left(z,\left(t^{\prime}-t^{\prime \prime}, h^{\prime \prime}\right)\right)
$$

which contradicts Theorem 4.11 (3).

The following result is an immediate consequence of Theorem 1.3.

Corollary 4.13. Let $\Omega$ be an X-Lipschitz domain with compact boundary in an equiregular CC space $\left(\mathbb{R}^{n}, X\right)$. Then the $X$-perimeter measure $|\partial \Omega|_{X}$ is doubling, i.e., there exists $C>0$ such that

$$
\frac{|\partial \Omega|_{X}(B(z, 2 r))}{|\partial \Omega|_{X}(B(z, r))} \leqslant C \quad \text { for any } z \in \partial \Omega, r>0 .
$$

A second interesting consequence of Theorem 1.3 is the following result, that obviously could be stated for both $\mathcal{H}_{d}^{Q-1}$ and $\mathcal{S}_{d}^{Q-1}$. We opted for the formulation with the spherical Hausdorff measure because it is the measure that more naturally arises when trying to represent the $X$-perimeter in terms of Hausdorff measures (see e.g. [21,33-35,44]). A very close result has been proved in [1].

Corollary 4.14. Let $\Omega$ be an X-Lipschitz domain with compact boundary in an equiregular $C C$ space $\left(\mathbb{R}^{n}, X\right)$. Then there exists $C=C(\Omega)$ such that

$$
\frac{1}{C} \mathcal{S}_{d}^{Q-1}\left\llcorner\partial \Omega \leqslant|\partial \Omega|_{X} \leqslant C \mathcal{S}_{d}^{Q-1}\llcorner\partial \Omega .\right.
$$

In particular, there exists a $\mathcal{S}_{d}^{Q-1}$-measurable function $\theta: \partial \Omega \rightarrow\left[\frac{1}{C}, C\right]$ such that $|\partial \Omega|_{X}=\theta \mathcal{S}_{d}^{Q-1}\llcorner\partial \Omega$.

Proof. The first part of the statement is an immediate consequence of (1.1) and of classical theorems on densities of measures, see e.g. [55, Theorem 3.2]. The second part follows from the Radon-Nikodym theorem. sequel.

We conclude this section with two further results that will be useful in the

Lemma 4.15. Let $\Omega$ be an $X$-Lipschitz domain in an equiregular CC space $\left(\mathbb{R}^{n}, X\right)$ and $x \in \partial \Omega$. Then there exist positive constants $C, R$ such that

$$
\mathcal{L}^{n}(\Omega \cap B(x, r)) \geqslant C r^{Q} \quad \text { for any } r \in(0, R) .
$$


Proof. Let $U, \mathcal{I}$ and $r_{0}$ be as in (4.21). Up to restricting $U$ we may find a Lipschitz function $f: U \rightarrow \mathbb{R}$ and $j \in\{1, \ldots, m\}$ such that

- $\Omega \cap U=\{f>0\}$ or $\Omega \cap U=\{f<0\}$;

- $f \in C^{\infty}(U \backslash \partial \Omega)$ and $l:=\inf _{U \backslash \partial \Omega} X_{j} f>0$.

We assume to fix ideas that $\Omega \cap U=\{f>0\}$ and give only some hint about how to adapt the argument to the case $\Omega \cap U=\{f<0\}$. Up to restricting $r_{0}$ we have $B\left(x, r_{0}\right) \subset U$. Since $d(\mathcal{I})$ is minimal we may assume that $Y_{i_{1}}=X_{j}$, so that $\exp ^{*}\left(t Y_{i_{1}}\right)=\exp \left(t X_{j}\right)$.

Let $L$ be the Lipschitz constant of $f$ and $\alpha$ be given by Theorem 4.11 (with $K:=\bar{U})$. Consider $r<R:=r_{0}$ and set $\delta:=\min \left\{\frac{\alpha l}{3 L}, \frac{\alpha}{5}\right\}$. We claim that

$$
E_{\mathcal{I}}(x, h) \in \Omega \cap B(x, r)
$$

whenever $h=\left(h_{1}, h^{\prime}\right) \in \mathbb{R} \times \mathbb{R}^{n-1}$ is such that $\left\|h^{\prime}\right\|_{\mathcal{I}}:=\left\|\left(0, h^{\prime}\right)\right\|_{\mathcal{I}}<\delta r$ and $h_{1} \in$ $(r / 2,3 r / 4)$ (if $\Omega \cap U=\{f<0\}$ we should instead consider $h_{1} \in(-r / 2,-3 r / 4)$ ). This would be enough to conclude because by Theorem 4.11 (1), (3)

$$
\begin{aligned}
\mathcal{L}^{n}(\Omega \cap B(x, r)) & \geqslant \int_{\left\{h \in \mathbb{R}^{n}: h_{1} \in(r / 2,3 r / 4),\left\|h^{\prime}\right\|_{\mathcal{I}<\delta r\}}\right.}\left|J_{h} E_{\mathcal{I}}(x, h)\right| d \mathcal{L}^{n} \\
& \geqslant C_{1} \mathcal{L}^{n}\left\{h \in \mathbb{R}^{n}: h_{1} \in(r / 2,3 r / 4),\left\|h^{\prime}\right\|_{\mathcal{I}}<\delta r\right\}=C_{2} r^{Q} .
\end{aligned}
$$

Let us prove (4.28). We have $E_{\mathcal{I}}\left(x,\left(0, h^{\prime}\right)\right) \in \mathcal{B}(x, \delta r) \subset B(x, \delta r / \alpha)$ and so

$$
\begin{aligned}
d\left(x, E_{\mathcal{I}}(x, h)\right) & \leqslant d\left(x, E_{\mathcal{I}}\left(x,\left(0, h^{\prime}\right)\right)\right)+d\left(E_{\mathcal{I}}\left(x,\left(0, h^{\prime}\right)\right), \exp \left(h_{1} X_{j}\right) E_{\mathcal{I}}\left(x,\left(0, h^{\prime}\right)\right)\right) \\
& <\frac{\delta r}{\alpha}+\left|h_{1}\right|<\left(\frac{1}{5}+\frac{3}{4}\right) r<r .
\end{aligned}
$$

Thus $E_{\mathcal{I}}(x, h) \in B\left(x, r_{0}\right) \subset \mathcal{Q}$ and (4.28) follows if we show that $f\left(E_{\mathcal{I}}(x, h)\right)>$ 0 (if $\Omega \cap U=\{f<0\}$ one would prove that $f\left(E_{\mathcal{I}}(x, h)\right)<0$ ). We have

$$
f\left(E_{\mathcal{I}}\left(x,\left(0, h^{\prime}\right)\right)\right) \geqslant f(x)-L \frac{\delta r}{\alpha}=-L \frac{\delta r}{\alpha},
$$

and by Lemma 2.2 (whose assumptions can be easily checked)

$$
\begin{aligned}
f\left(E_{\mathcal{I}}(x, h)\right) & =f\left(\exp \left(h_{1} X_{j}\right) E_{\mathcal{I}}\left(x,\left(0, h^{\prime}\right)\right)\right) \\
& \geqslant f\left(E_{\mathcal{I}}\left(x,\left(0, h^{\prime}\right)\right)\right)+l h_{1} \geqslant-L \frac{\delta r}{\alpha}+l \frac{r}{2} \geqslant l r / 6>0 .
\end{aligned}
$$

It follows that $E_{\mathcal{I}}(x, h) \in \Omega \cap B(z, r)$ and (4.28) follows.

Lemma 4.16. Let $\Omega$ be an $X$-Lipschitz domain with compact boundary in an equiregular CC space $\left(\mathbb{R}^{n}, X\right)$ and $\mu$ a finite Borel measure in $\Omega$. Then

$$
\lim _{r \rightarrow 0^{+}} \frac{\mu(B(x, r) \cap \Omega)}{r^{Q-1}}=0 \quad \text { for }|\partial \Omega|_{X} \text {-a.e. } x \in \partial \Omega \text {. }
$$


Proof. By virtue of (1.1), it is enough to prove that

$$
\lim _{r \rightarrow 0^{+}} \frac{\mu(B(x, r) \cap \Omega)}{|\partial \Omega|_{X}(B(x, r))}=0 \quad \text { for }|\partial \Omega|_{X} \text {-a.e. } x \in \partial \Omega .
$$

In turn, it is sufficient to show that $|\partial \Omega|_{X}\left(A_{k}\right)=0$ for any $k \in \mathbb{N}$, where

$$
A_{k}:=\left\{x \in \partial \Omega: \limsup _{r \rightarrow 0^{+}} \frac{\mu(B(x, r) \cap \Omega)}{|\partial \Omega|_{X}(B(x, r))}>\frac{1}{k}\right\} .
$$

Let $\epsilon>0$ be fixed; by Vitali covering theorem (see e.g. [55, Theorem 3.3]) there exist $\left(x_{i}\right) \subset A_{k}$ and $r_{i}<\epsilon$ such that

$$
\begin{aligned}
& \frac{\mu\left(B\left(x, r_{i}\right) \cap \Omega\right)}{|\partial \Omega|_{X}\left(B\left(x, r_{i}\right)\right)}>\frac{1}{k} \text { for any } i \\
& B\left(x_{i}, r_{i}\right) \text { are pairwise disjoint and } A_{k} \subset \bigcup_{i} B\left(x_{i}, 5 r_{i}\right) .
\end{aligned}
$$

By Theorem 1.3

$$
\begin{aligned}
|\partial \Omega|_{X}\left(A_{k}\right) & \leqslant \sum_{i}|\partial \Omega|_{X}\left(B\left(x_{i}, 5 r_{i}\right)\right) \leqslant \sum_{i} 5^{Q-1} \lambda r_{i}^{Q-1} \\
& \leqslant 5^{Q-1} \lambda^{2} \sum_{i}|\partial \Omega|_{X}\left(B\left(x_{i}, r_{i}\right)\right) \leqslant 5^{Q-1} \lambda^{2} k \mu\left(\Omega \cap \bigcup_{i} B\left(x_{i}, r_{i}\right)\right) \\
& \leqslant 5^{Q-1} \lambda^{2} k \mu\left((\partial \Omega)_{\epsilon}\right)
\end{aligned}
$$

where $(\partial \Omega)_{\epsilon}:=\{z \in \Omega: d(z, \partial \Omega)<\epsilon\}$. The desired equality $|\partial \Omega|_{X}\left(A_{k}\right)=0$ now follows because $\mu\left((\partial \Omega)_{\epsilon}\right) \rightarrow 0$ as $\epsilon \rightarrow 0$.

\section{Trace theorems for $B V_{X}$ functions on $X$-Lipschitz domains}

The theory of traces for Euclidean BV functions is well established; for a more general account on this subject, we address the interested reader to $[4,46,47]$ and the monographs $[2,41]$.

\subsection{Existence of traces}

We begin this section by proving the existence of traces for $B V_{X}$ functions on $X$ Lipschitz domains with compact boundary.

Proof of Theorem 1.4. We claim that for any $x \in \partial \Omega$ there exist $C_{x}>0$ and a neighbourhood $\mathcal{Q}=\mathcal{Q}_{x}$ such that the following holds. For any $u \in B V_{X}(\Omega)$ such 
that $\{u \neq 0\} \Subset \mathcal{Q}$, there exists $T u \in L^{1}\left(\partial \Omega,|\partial \Omega|_{X}\right)$ such that

(1.2) holds for any $g \in C^{1}\left(\mathbb{R}^{n}, \mathbb{R}^{m}\right)$;

$T u=0$ on $\partial \Omega \backslash \mathcal{Q}$;

$u \mapsto T u$ is linear on the vector space $\left\{u \in B V_{X}(\Omega):\{u \neq 0\} \Subset \mathcal{Q}\right\}$;

$\|T u\|_{L^{1}\left(\partial \Omega,|\partial \Omega|_{X}\right)} \leqslant C_{x}|X u|(\Omega)$.

This would be enough to conclude. Indeed, since $\partial \Omega$ is compact we can find $x_{1}, \ldots, x_{q} \in \partial \Omega$, together with the associated neighbourhood $\mathcal{Q}_{1}, \ldots, \mathcal{Q}_{q}\left(\mathcal{Q}_{i}:=\right.$ $\mathcal{Q}_{x_{i}}$ ), such that $\partial \Omega \subset \bigcup_{i=1}^{q} \mathcal{Q}_{i}$. We also consider an open set $\mathcal{Q}_{0}$ with $\Omega \backslash$ $\bigcup_{i=1}^{q} \mathcal{Q}_{i} \subset \mathcal{Q}_{0} \Subset \Omega$. Let $\left(\psi_{i}\right)_{i=0, \ldots, q}$ be smooth functions such that

$$
\psi_{i} \in C_{c}^{\infty}\left(\mathcal{Q}_{i}\right), \quad 0 \leqslant \psi_{i} \leqslant 1, \quad \sum_{i=0}^{q} \psi_{i}=1 \text { on } \Omega .
$$

Thus, for a generic $u \in B V_{X}(\Omega)$ we have $\psi_{i} u \in B V_{X}(\Omega),\left\{\psi_{i} u \neq 0\right\} \Subset \mathcal{Q}_{i}$ and

$$
X\left(\psi_{i} u\right)=u\left(X \psi_{i}\right) \mathcal{L}^{n}+\psi_{i} X u \quad \text { on } \Omega
$$

in the sense of distributions. Let $g \in C^{1}\left(\mathbb{R}^{n}, \mathbb{R}^{m}\right)$ be fixed; our claim implies that for any $i=1, \ldots, q$

$$
\begin{aligned}
& \int_{\Omega}\left(\psi_{i} u\right) \operatorname{div}_{X} g d \mathcal{L}^{n} \\
= & -\int_{\Omega} \psi_{i}\left\langle\sigma_{u}, g\right\rangle d|X u|-\int_{\Omega} u\left\langle X \psi_{i}, g\right\rangle d \mathcal{L}^{n}+\int_{\partial \Omega}\left\langle\nu_{\Omega}, g\right\rangle T\left(\psi_{i} u\right) d|\partial \Omega|_{X}
\end{aligned}
$$

while

$$
\int_{\Omega}\left(\psi_{0} u\right) \operatorname{div}_{X} g d \mathcal{L}^{n}=-\int_{\Omega} \psi_{0}\left\langle\sigma_{u}, g\right\rangle d|X u|-\int_{\Omega} u\left\langle X \psi_{0}, g\right\rangle d \mathcal{L}^{n} .
$$

Taking into account that $\sum_{i=0}^{q} X \psi_{i}=0$ on $\Omega$, on summing (5.6) (for $i=1, \ldots, q$ ) and (5.7) we obtain (1.2) with $T u:=\sum_{i=1}^{q} T\left(\psi_{i} u\right)$.

The linearity of the trace operator $T$ stems from (5.3). As for the boundedness of $T$, we have from (5.4) that for any $i=1, \ldots, q$

$$
\left\|T\left(\psi_{i} u\right)\right\|_{L^{1}\left(\partial \Omega,|\partial \Omega|_{X}\right)} \leqslant C_{x_{i}}\left|X\left(\psi_{i} u\right)\right|(\Omega) \leqslant C_{x_{i}}^{\prime}\left[|X u|(\Omega)+\|u\|_{L^{1}(\Omega)}\right],
$$

where we have used the fact that, by (5.5) and $\left|\psi_{i}\right| \leqslant 1$,

$$
\left|X\left(\psi_{i} u\right)\right|(\Omega) \leqslant\left\|X \psi_{i}\right\|_{L^{\infty}}\|u\|_{L^{1}(\Omega)}+|X u|(\Omega) .
$$

From (5.8) it is easy to get the existence of $C=C(\Omega)$ such that

$$
\|T u\|_{L^{1}\left(\partial \Omega,|\partial \Omega|_{X}\right)} \leqslant C\left[|X u|(\Omega)+\|u\|_{L^{1}(\Omega)}\right] .
$$

This would conclude the proof. 
For the reader's convenience, we divide the proof of the claim into several steps.

Step 1. Let $x \in \partial \Omega$ be fixed. By Lemma 4.3, there exist a neighbourhood $U=U_{x}$, a Lipschitz function $f: U \rightarrow \mathbb{R}$ and $j \in\{1, \ldots, m\}$ such that

- $\Omega \cap U=\{f>0\}$ or $\Omega \cap U=\{f<0\}$;

- $f \in C^{\infty}(U \backslash \partial \Omega)$ and $l:=\inf _{U \backslash \partial \Omega} X_{j} f>0$.

We assume that $\Omega \cap U=\{f>0\}$; we will treat the case $\Omega \cap U=\{f<0\}$ later in Step 3. Let then $\mathcal{Q}_{x}=\mathcal{Q} \subset U$ be the open neighbourhood given by Proposition 4.5 (applied to $S:=\partial \Omega \cap U$ ). We will also consider

$$
I, a, G, \phi, \phi_{\epsilon}, \Phi, \Phi_{\epsilon}, \tilde{f}, \Omega_{\epsilon}, \widetilde{\Omega}, \widetilde{\Omega}_{\epsilon}, \widetilde{X}_{i}, \widetilde{X}, \rho, \rho_{\epsilon}
$$

as in Proposition 4.5 and its proof.

Let $u \in B V_{X}(\Omega)$ be such that $\{u \neq 0\} \Subset \mathcal{Q}$. We initially work under the additional assumption $u \in C^{\infty}(\Omega \cap \mathcal{Q})$. Let $g \in C^{1}\left(\mathbb{R}^{n}, \mathbb{R}^{m}\right)$ be fixed; since $\partial \Omega_{\epsilon}$ is smooth and $\Omega_{\epsilon}=\{f>\epsilon\}$ we have

$$
\begin{aligned}
\int_{\Omega} u \operatorname{div}_{X} g d \mathcal{L}^{n} & =\lim _{\epsilon \rightarrow 0^{+}} \int_{\Omega_{\epsilon} \cap \mathcal{Q}} u \operatorname{div}_{X} g d \mathcal{L}^{n} \\
& =\lim _{\epsilon \rightarrow 0^{+}}\left(-\int_{\Omega_{\epsilon} \cap \mathcal{Q}}\langle X u, g\rangle d \mathcal{L}^{n}+\int_{\partial \Omega_{\epsilon} \cap \mathcal{Q}} u\left\langle v_{\Omega_{\epsilon}}, g\right\rangle d\left|\partial \Omega_{\epsilon}\right| X\right) \\
& =-\int_{\Omega}\langle X u, g\rangle d \mathcal{L}^{n}+\lim _{\epsilon \rightarrow 0^{+}} \int_{I}\left(\tilde{u} \circ \Phi_{\epsilon}\right)\left\langle\tilde{g} \circ \Phi_{\epsilon}, \rho_{\epsilon}\right\rangle d \mathcal{L}^{n-1}
\end{aligned}
$$

where we have set $\tilde{u}:=u \circ G: I \times(-a, a) \rightarrow \mathbb{R}, \tilde{g}:=g \circ G: I \times(-a, a) \rightarrow \mathbb{R}$ and used (4.19). As in Proposition 4.5, we hereafter agree that $\epsilon$ is small enough, so that $\phi_{\epsilon}$ is defined on the whole $I$.

We claim that

$$
v_{\epsilon}:=\tilde{u} \circ \Phi_{\epsilon} \text { is a Cauchy sequence in } L^{1}(I) \text { as } \epsilon \rightarrow 0^{+} .
$$

Assume (5.10): then, there would exist $v \in L^{1}(I)$ such that $v_{\epsilon} \rightarrow v$ in $L^{1}(I)$ and, taking into account the uniform convergence $\Phi_{\epsilon} \rightarrow \Phi$ and the weak-* convergence $\rho_{\epsilon} \stackrel{*}{\rightarrow} \rho$ in $L^{\infty}\left(I, \mathbb{R}^{m}\right)$, one would get

$$
\begin{aligned}
\lim _{\epsilon \rightarrow 0^{+}} \int_{I}\left(\tilde{u} \circ \Phi_{\epsilon}\right)\left\langle\tilde{g} \circ \Phi_{\epsilon}, \rho_{\epsilon}\right\rangle d \mathcal{L}^{n-1} & =\int_{I} v\langle\tilde{g} \circ \Phi, \rho\rangle d \mathcal{L}^{n-1} \\
& =\int_{\partial \Omega} T u\left\langle g, v_{\Omega}\right\rangle d|\partial \Omega|_{X} .
\end{aligned}
$$

Here we have used (4.10) and defined $T u \in L^{1}\left(\partial \Omega,|\partial \Omega|_{X}\right)$ as

$$
T u= \begin{cases}v \circ \Phi^{-1} \circ G^{-1} & \text { on } \partial \Omega \cap \mathcal{Q} \\ 0 & \text { on } \partial \Omega \backslash \mathcal{Q}\end{cases}
$$


From (5.9) and (5.11) we would achieve

$$
\int_{\Omega} u \operatorname{div}_{X} g d \mathcal{L}^{n}=-\int_{\Omega}\langle X u, g\rangle d \mathcal{L}^{n}+\int_{\partial \Omega}\left\langle\nu_{\Omega}, g\right\rangle T u d|\partial \Omega|_{X}
$$

and (5.1) would be proved for such $u$.

Let us prove (5.10). For $y \in I$ and $0<\delta<\epsilon$ we have

$$
v_{\epsilon}(y)-v_{\delta}(y)=\tilde{u}\left(y, \phi_{\epsilon}(y)\right)-\tilde{u}\left(y, \phi_{\delta}(y)\right)=\int_{\phi_{\delta}(y)}^{\phi_{\epsilon}(y)}\left(\partial_{s_{n}} \tilde{u}\right)(y, t) d t .
$$

Setting

$$
R_{\epsilon}:=\left\{(y, t): y \in I, \phi(y)<t<\phi_{\epsilon}(y)\right\}=\{0<\tilde{f}<\epsilon\}
$$

from (5.12) we obtain

$$
\begin{aligned}
\int_{I}\left|v_{\epsilon}(y)-v_{\delta}(y)\right| d y & \leqslant \int_{I} \int_{\phi_{\delta}(y)}^{\phi_{\epsilon}(y)}\left|\tilde{X}_{j} \tilde{u}(y, t)\right| d t d y \leqslant \int_{R_{\epsilon}}|\tilde{X} \tilde{u}| d \mathcal{L}^{n} \\
& =\int_{G\left(R_{\epsilon}\right)}|X u|\left|\operatorname{det} J G^{-1}\right| d \mathcal{L}^{n} \leqslant C_{1} \int_{G\left(R_{\epsilon}\right)}|X u| d \mathcal{L}^{n}
\end{aligned}
$$

Then, (5.10) follows thanks to the absolute integrability of $|X u|$ and the fact that $\bigcap_{\epsilon>0} G\left(R_{\epsilon}\right)=\emptyset$.

We observe also that (5.2) and (5.3) are straightforward (of course, the linearity of $T$ in (5.3) is for the moment understood on the subspace $\left\{u \in B V_{X}(\Omega) \cap C^{\infty}(\Omega \cap\right.$ $\mathcal{Q}):\{u \neq 0\} \Subset \mathcal{Q}\})$, while

$$
v_{\epsilon}(y)=-\int_{\phi_{\epsilon}(y)}^{a}\left(\partial_{s_{n}} \tilde{u}\right)(y, t) d t \quad \forall y \in I
$$

gives the inequality

$$
\begin{aligned}
\int_{I}|v| d \mathcal{L}^{n-1} & =\lim _{\epsilon \rightarrow 0^{+}} \int_{I}\left|v_{\epsilon}\right| d \mathcal{L}^{n-1} \leqslant \int_{I \times(-a, a) \backslash R_{\epsilon}}|\tilde{X} \tilde{u}| d \mathcal{L}^{n} \\
& =\int_{G\left(R_{\epsilon}\right)}|X u|\left|\operatorname{det} J G^{-1}\right| d \mathcal{L}^{n} \leqslant C_{1}|X u|(\Omega),
\end{aligned}
$$

whence (5.4).

Step 2. We now consider the case of a generic function $u \in B V_{X}(\Omega)$ such that $\{u \neq 0\} \Subset \mathcal{Q}$. Let us fix a sequence $\left(u_{k}\right)_{k} \subset B V_{X}(\Omega) \cap C^{\infty}(\Omega)$ such that

$$
u_{k} \rightarrow u \text { in } L^{1}(\Omega) \quad \text { and } \quad\left|X u_{k}\right|(\Omega) \rightarrow|X u|(\Omega) .
$$

It is not restrictive to assume that $\left\{u_{k} \neq 0\right\} \Subset \mathcal{Q}$; this is possible because the approximating sequence $\left(u_{k}\right)$ is essentially obtained by mollification, see the proof of [31, Theorem 2.2.2] for more details. 
We claim that $\left(T u_{k}\right)_{k}$ is a Cauchy sequence in $L^{1}\left(\partial \Omega,|\partial \Omega|_{X}\right)$. This would be sufficient to conclude: in fact, after setting $T u:=L^{1}-\lim _{k \rightarrow \infty} T u_{k} \in L^{1}\left(\partial \Omega,|\partial \Omega|_{X}\right)$, on passing to the limit as $k \rightarrow \infty$ in the equality

$$
\int_{\Omega} u_{k} \operatorname{div}_{X} g d \mathcal{L}^{n}=-\int_{\Omega}\left\langle X u_{k}, g\right\rangle d \mathcal{L}^{n}+\int_{\partial \Omega}\left\langle v_{\Omega}, g\right\rangle T u_{k} d|\partial \Omega|_{X}
$$

we obtain (5.1). Statements (5.2), (5.3) and (5.4) are straightforward.

Let us prove our claim or, equivalently, that the sequence $\left(v_{k}\right)_{k}, v_{k}:=\left(T u_{k}\right) \circ$ $G \circ \Phi$, is a Cauchy sequence in $L^{1}\left(I, \mathcal{L}^{n-1}\right)$. Set $\tilde{u}_{k}:=u_{k} \circ G: I \times(-a, a) \rightarrow \mathbb{R}$ and $v_{k, \epsilon}:=\tilde{u}_{k} \circ \Phi_{\epsilon}: I \rightarrow \mathbb{R}$; recall that $v_{k, \epsilon} \rightarrow v_{k}$ in $L^{1}(I)$ as $\epsilon \rightarrow 0^{+}$. From (5.13) we deduce

$$
\int_{I}\left|v_{k}-v_{k, \epsilon}\right| \mathcal{L}^{n-1} \leqslant C_{1}\left|X u_{k}\right|\left(G\left(R_{\epsilon}\right)\right)
$$

and, setting $v_{k}^{\epsilon}:=\frac{1}{\epsilon} \int_{0}^{\epsilon} v_{k, t} d t$, one gets

$$
\int_{I}\left|v_{k}-v_{k}^{\epsilon}\right| d \mathcal{L}^{n-1} \leqslant \frac{1}{\epsilon} \int_{I} \int_{0}^{\epsilon}\left|v_{k}-v_{k, t}\right| d t d \mathcal{L}^{n-1} \leqslant C_{1}\left|X u_{k}\right|\left(G\left(R_{\epsilon}\right)\right) .
$$

Therefore

$$
\begin{aligned}
& \int_{I}\left|v_{k}-v_{\ell}\right| d \mathcal{L}^{n-1} \\
\leqslant & \int_{I}\left|v_{k}-v_{k}^{\epsilon}\right| d \mathcal{L}^{n-1}+\int_{I}\left|v_{\ell}-v_{\ell}^{\epsilon}\right| d \mathcal{L}^{n-1}+\int_{I}\left|v_{k}^{\epsilon}-v_{\ell}^{\epsilon}\right| d \mathcal{L}^{n-1} \\
\leqslant & C_{1}\left|X u_{k}\right|\left(G\left(R_{\epsilon}\right)\right)+C_{1}\left|X u_{\ell}\right|\left(G\left(R_{\epsilon}\right)\right)+\frac{1}{\epsilon} \int_{0}^{\epsilon} \int_{I}\left|v_{k, t}-v_{\ell, t}\right| d \mathcal{L}^{n-1} d t .
\end{aligned}
$$

Taking into account that inf $\left\{\left|\rho_{t}(y)\right|: y \in I, t \in(0, \epsilon)\right\}>0$ (see Remark 4.8), by (4.19) one achieves

$$
\begin{aligned}
\frac{1}{\epsilon} \int_{0}^{\epsilon} \int_{I}\left|v_{k, t}-v_{\ell, t}\right| d \mathcal{L}^{n-1} d t & \leqslant \frac{C_{2}}{\epsilon} \int_{0}^{\epsilon} \int_{I}\left|\tilde{u}_{k} \circ \Phi_{t}-\tilde{u}_{\ell} \circ \Phi_{t}\right|\left|\rho_{t}\right| d \mathcal{L}^{n-1} d t \\
& =\frac{C_{2}}{\epsilon} \int_{0}^{\epsilon} \int_{\partial \Omega_{t} \cap \mathcal{Q}}\left|u_{k}-u_{\ell}\right| d\left|\partial \Omega_{t}\right|_{X} d t \\
& =\frac{C_{2}}{\epsilon} \int_{G\left(R_{\epsilon}\right)}\left|u_{k}-u_{\ell}\right||X f| d \mathcal{L}^{n},
\end{aligned}
$$

where, in the last equality, we have used Theorem 2.6. By (5.15) we obtain

$$
\begin{gathered}
\int_{I}\left|v_{k}-v_{\ell}\right| d \mathcal{L}^{n-1} \leqslant C_{1}\left|X u_{k}\right|\left(G\left(R_{\epsilon}\right)\right)+C_{1}\left|X u_{\ell}\right|\left(G\left(R_{\epsilon}\right)\right) \\
+\frac{C_{3}}{\epsilon} \int_{G\left(R_{\epsilon}\right)}\left|u_{k}-u_{\ell}\right| d \mathcal{L}^{n} \\
\leqslant C_{1}\left|X u_{k}\right|\left(K_{\epsilon}\right)+C_{1}\left|X u_{\ell}\right|\left(K_{\epsilon}\right)+\frac{C_{3}}{\epsilon} \int_{G\left(R_{\epsilon}\right)}\left|u_{k}-u_{\ell}\right| d \mathcal{L}^{n}
\end{gathered}
$$


where $K_{\epsilon}:=\overline{G\left(R_{\epsilon}\right)} \cap \Omega \subset \overline{\mathcal{Q}} \cap \Omega$. By Lemma 2.4 we get

$$
\limsup _{k, \ell \rightarrow \infty} \int_{I}\left|v_{k}-v_{\ell}\right| d \mathcal{L}^{n-1} \leqslant 2 C_{1}|X u|\left(K_{\epsilon}\right) \quad \forall \epsilon>0 .
$$

Since $\mathcal{Q} \cap \bigcap_{\epsilon} K_{\epsilon}=\emptyset$ we have $\bigcap_{\epsilon} K_{\epsilon} \subset \partial \mathcal{Q} \cap \Omega$ whence

$$
|X u|\left(\bigcap_{\epsilon} K_{\epsilon}\right) \leqslant|X u|(\partial \mathcal{Q} \cap \Omega)=0
$$

because $u=0$ in a neighbourhood of $\partial \mathcal{Q} \cap \Omega$. From (5.17) we get

$$
\limsup _{k, \ell \rightarrow \infty} \int_{I}\left|v_{k}-v_{\ell}\right| d \mathcal{L}^{n-1}=0
$$

and our claim follows.

For future references we also observe what follows. If we set $v_{\epsilon}:=\tilde{u} \circ \Phi_{\epsilon}$ : $I \rightarrow \mathbb{R}$ and $v^{\epsilon}:=\frac{1}{\epsilon} \int_{0}^{\epsilon} v_{t} d t$, we have

$\int_{I}\left|v_{k}^{\epsilon}-v^{\epsilon}\right| d \mathcal{L}^{n-1} \leqslant \frac{1}{\epsilon} \int_{0}^{\epsilon} \int_{I}\left|v_{k, t}-v_{t}\right| d \mathcal{L}^{n-1} d t \leqslant \frac{C_{2}}{\epsilon} \int_{G\left(R_{\epsilon}\right)}\left|u_{k}-u\right||X f| d \mathcal{L}^{n}$, where the second inequality may be justified just like (5.16). This implies that for any $\epsilon$

$$
v_{k}^{\epsilon} \rightarrow v^{\epsilon} \text { in } L^{1}(I) \text { as } k \rightarrow \infty .
$$

Since $v_{k} \rightarrow v$ in $L^{1}(I)$, from (5.14) and (5.18) we obtain as $k \rightarrow \infty$

$$
\int_{I}\left|v-v^{\epsilon}\right| d \mathcal{L}^{n-1} \leqslant C_{1}|X u|\left(K_{\epsilon}\right)
$$

where we have used Lemma 2.4 again.

Step 3. We now go back to Step 1 and analyze the case $\Omega \cap U=\{f<0\}$. Set $\hat{f}=$ $-f$ and $\hat{X}=\left(X_{1}, \ldots, X_{j-1},-X_{j}, X_{j+1}, \ldots, X_{m}\right)$; notice that $|\partial \Omega|_{\hat{X}}=|\partial \Omega|_{X}$, $|\partial \Omega|_{\hat{X}}$ denoting the perimeter measure of $\Omega$ induced by the family $\hat{X}$. Then

$$
\Omega=\{\hat{f}>0\}, \quad \hat{f} \in C^{\infty}(U \backslash \partial \Omega) \quad \text { and } \quad l:=\inf _{U \backslash \partial \Omega} \hat{X}_{j} \hat{f}>0 .
$$

Reasoning as in Steps 1 and 2 we can find $C>0$ and a neighbourhood $\mathcal{Q}$ of $x$ such that the following holds. For any $u \in B V_{X}(\Omega)=B V_{\hat{X}}(\Omega)$ with $\{u \neq 0\} \Subset \mathcal{Q}$ there exists $\hat{T} u \in L^{1}\left(\partial \Omega,|\partial \Omega|_{\hat{X}}\right)=L^{1}\left(\partial \Omega,|\partial \Omega|_{X}\right)$ such that

$$
\begin{aligned}
& \text { for any } g \in C^{1}\left(\mathbb{R}^{n}, \mathbb{R}^{m}\right) \\
& \qquad \int_{\Omega} u \operatorname{div}_{\hat{X}} g d \mathcal{L}^{n}=-\int_{\Omega}\left\langle\hat{\sigma}_{u}, g\right\rangle d|\hat{X} u|+\int_{\partial \Omega}\left\langle\hat{\nu}_{\Omega}, g\right\rangle \hat{T} u d|\partial \Omega|_{\hat{X}} ; \\
& \hat{T} u=0 \text { on } \partial \Omega \backslash \mathcal{Q} ; \\
& u \mapsto \hat{T} u \text { is linear on the vector space }\left\{u \in B V_{\hat{X}}(\Omega):\{u \neq 0\} \Subset \mathcal{Q}\right\} ; \\
& \|\hat{T} u\|_{L^{1}\left(\partial \Omega,|\partial \Omega|_{\hat{X}}\right)} \leqslant C|\hat{X} u|(\Omega) \text {. }
\end{aligned}
$$


Here, $\hat{\sigma}_{u}$ and $\hat{\nu}_{\Omega}$ are such that $\hat{X} u=\hat{\sigma}_{u}|\hat{X} u|$ and $\hat{X} \chi_{\Omega}=\hat{v}_{\Omega}|\partial \Omega|_{\hat{X}}$. It is easily seen that $|\hat{X} u|=|X u|$ and

$$
\begin{aligned}
& \hat{\sigma}_{u}=\left(\left(\sigma_{u}\right)_{1}, \ldots,-\left(\sigma_{u}\right)_{j}, \ldots,\left(\sigma_{u}\right)_{m}\right) \\
& \hat{v}_{\Omega}=\left(\left(v_{\Omega}\right)_{1}, \ldots,-\left(v_{\Omega}\right)_{j}, \ldots,\left(v_{\Omega}\right)_{m}\right),
\end{aligned}
$$

whence (5.1), (5.2), (5.3) and (5.4) follow (with $T u:=\hat{T} u)$, respectively, from (5.20), (5.21), (5.22) and (5.23). This concludes the proof.

Remark 5.1. If $u \in C^{0}(\bar{\Omega}) \cap B V_{X}(\Omega)$, then $T u=u_{\mid \partial \Omega}$. Using the same notation of the previous proof, it suffices to notice that $T\left(\psi_{i} u\right)=\psi_{i} u$ for any $i=1, \ldots, q$ because of (5.19).

Remark 5.2. Under the assumptions of Theorem 1.4: the trace operator $T$ is local, i.e., if $u_{1}, u_{2} \in B V_{X}(\Omega)$ are such that $u_{1}=u_{2}$ on some open set $U$, then $\left(T u_{1}\right)_{\mid \partial \Omega \cap U}=\left(T u_{2}\right)_{\mid \partial \Omega \cap U}$.

When $\Omega \subset \mathbb{R}^{n}$ is an $X$-Lipschitz domain with compact boundary and $u \in$ $L^{1}\left(\mathbb{R}^{n}\right)$ is such that

$$
u_{\mid \Omega} \in B V_{X}(\Omega) \quad \text { and } \quad u_{\mid \mathbb{R}^{n} \backslash \bar{\Omega}} \in B V_{X}\left(\mathbb{R}^{n} \backslash \bar{\Omega}\right)
$$

one can define the "inner" and "outer" traces $T^{+} u, T^{-} u \in L^{1}\left(\partial \Omega,|\partial \Omega|_{X}\right)$ of $u$ on $\partial \Omega$, respectively, as

$$
T^{+} u:=T^{\Omega}\left(u_{\mid \Omega}\right), \quad T^{-} u:=T^{\mathbb{R}^{n} \backslash \bar{\Omega}}\left(u_{\mid \mathbb{R}^{n} \backslash \bar{\Omega}}\right) .
$$

We denoted by $T^{\Omega}, T^{\mathbb{R}^{n} \backslash \bar{\Omega}}$, respectively, the trace operators

$$
\begin{aligned}
& T^{\Omega}: B V_{X}(\Omega) \rightarrow L^{1}\left(\partial \Omega,|\partial \Omega|_{X}\right) \\
& T^{\mathbb{R}^{n} \backslash \bar{\Omega}}: B V_{X}\left(\mathbb{R}^{n} \backslash \bar{\Omega}\right) \rightarrow L^{1}\left(\partial\left(\mathbb{R}^{n} \backslash \bar{\Omega}\right),\left|\partial\left(\mathbb{R}^{n} \backslash \bar{\Omega}\right)\right|_{X}\right) \equiv L^{1}\left(\partial \Omega,|\partial \Omega|_{X}\right) .
\end{aligned}
$$

We are going to prove that such a $u$ is actually of bounded $X$-variation on the whole $\mathbb{R}^{n}$; we will also characterize the measure $X u$.

Theorem 5.3. Let $\Omega \subset \mathbb{R}^{n}$ be an $X$-Lipschitz domain with compact boundary and $u \in L^{1}\left(\mathbb{R}^{n}\right)$ such that $u_{\mid \Omega} \in B V_{X}(\Omega)$ and $u_{\mid \mathbb{R}^{n} \backslash \bar{\Omega}} \in B V_{X}\left(\mathbb{R}^{n} \backslash \bar{\Omega}\right)$. Then $u \in$ $B V_{X}\left(\mathbb{R}^{n}\right)$ and

$$
X u\left\llcorner\partial \Omega=\left(T^{+} u-T^{-} u\right) v_{\Omega}|\partial \Omega|_{X}\right.
$$

In particular, $X u=X u\left\llcorner\Omega+X u\left\llcorner\left(\mathbb{R}^{n} \backslash \bar{\Omega}\right)+\left(T^{+} u-T^{-} u\right) \nu_{\Omega}|\partial \Omega|_{X}\right.\right.$. 
Proof. For any $g \in C_{c}^{1}\left(\mathbb{R}^{n}, \mathbb{R}^{m}\right)$ with $|g| \leqslant 1$ we have by Theorem 1.4 and Corollary 4.2

$$
\begin{aligned}
\int_{\mathbb{R}^{n}} u \operatorname{div}_{X} g d \mathcal{L}^{n}= & \int_{\mathbb{R}^{n} \cap \Omega} u \operatorname{div}_{X} g d \mathcal{L}^{n}+\int_{\mathbb{R}^{n} \backslash \bar{\Omega}} u \operatorname{div}_{X} g d \mathcal{L}^{n} \\
= & -\int_{\mathbb{R}^{n} \cap \Omega}\left\langle\sigma_{u}, g\right\rangle d|X u|+\int_{\partial \Omega}\left\langle v_{\Omega}, g\right\rangle T^{+} u d|\partial \Omega|_{X} \\
& -\int_{\mathbb{R}^{n} \backslash \bar{\Omega}}\left\langle\sigma_{u}, g\right\rangle d|X u|+\int_{\partial \Omega}\left\langle v_{\mathbb{R}^{n} \backslash \bar{\Omega}}, g\right\rangle T^{-} u d|\partial \Omega|_{X} \\
= & -\int_{\mathbb{R}^{n} \backslash \partial \Omega}\left\langle\sigma_{u}, g\right\rangle d|X u|+\int_{\partial \Omega}\left\langle v_{\Omega}, g\right\rangle\left(T^{+} u-T^{-} u\right) d|\partial \Omega|_{X}
\end{aligned}
$$

where we have used the fact that $v_{\mathbb{R}^{n} \backslash \bar{\Omega}}=-v_{\Omega}|\partial \Omega|_{X}$-a.e. This gives (5.24) and concludes the proof.

\subsection{Extension of $B V_{X}$ functions}

The most part of this section is devoted to the proof of Theorem 1.5.

Proof of Theorem 1.5. Let $\mathcal{Q}_{1}, \ldots, \mathcal{Q}_{q}$ be the open sets considered in the proof of Theorem 1.4. We will prove that, if $\delta>0$ and $w$ is such that

$$
\text { spt } w \subset \partial \Omega \cap \mathcal{Q}_{i} \text { for a suitable } i,
$$

then there exists $u \in C^{\infty}(\Omega) \cap W_{X}^{1,1}(\Omega)$ such that (1.3) holds for some $C=C_{i}$ and, if $\partial \Omega$ is $X$-regular, (1.4) holds as well. This would be enough to conclude. Indeed, let $w$ be a generic function in $L^{1}\left(\partial \Omega,|\partial \Omega|_{X}\right)$ and consider functions $\psi_{i}$ such that

$$
\psi_{i} \in C_{c}^{\infty}\left(\mathcal{Q}_{i}\right), \quad 0 \leqslant \psi_{i} \leqslant 1, \quad \sum_{i=1}^{q} \psi_{i}=1 \text { on } \partial \Omega
$$

Then for any $i=1, \ldots, q$ we could choose $u_{i} \in C^{\infty}(\Omega) \cap W_{X}^{1,1}(\Omega)$ such that

$$
T u_{i}=\psi_{i} w, \quad \int_{\Omega}\left|u_{i}\right| d \mathcal{L}^{n} \leqslant \frac{\delta}{q}, \quad \int_{\Omega}\left|X u_{i}\right| d \mathcal{L}^{n} \leqslant C_{i}\left\|\psi_{i} w\right\|_{L^{1}\left(\partial \Omega,|\partial \Omega|_{X}\right)}
$$

and (1.3) would follow, with $u:=u_{1}+\cdots+u_{q}$ and $C:=\sup _{i} C_{i}$, because $\psi_{i}$ are nonnegative and thus

$$
\|w\|_{L^{1}\left(\partial \Omega,|\partial \Omega|_{X}\right)}=\left\|\psi_{1} w\right\|_{L^{1}\left(\partial \Omega,|\partial \Omega|_{X}\right)}+\cdots+\left\|\psi_{q} w\right\|_{L^{1}\left(\partial \Omega,|\partial \Omega|_{X}\right)} .
$$

If $\Omega$ is $X$-regular, (1.4) follows provided we choose $u_{i}$ such that

$$
\int_{\Omega}\left|X u_{i}\right| d \mathcal{L}^{n} \leqslant(1+\delta)\left\|\psi_{i} w\right\|_{L^{1}\left(\partial \Omega,|\partial \Omega|_{X}\right)} .
$$


Step 1. Let $i \in\{1, \ldots, q\}$ and $w \in L^{1}\left(\partial \Omega,|\partial \Omega|_{X}\right)$ with spt $w \subset \mathcal{Q}:=\mathcal{Q}_{i}$ be fixed. Let $f, j$ and $l$ be as in the proof of Theorem 1.4, Step 1. We first assume that $\Omega \cap \mathcal{Q}=\{f>0\}$ and define

$$
I, a, G, \phi, \phi_{\epsilon}, \Phi, \Phi_{\epsilon}, \tilde{f}, \Omega_{\epsilon}, \widetilde{\Omega}, \widetilde{\Omega}_{\epsilon}, \tilde{X}_{\ell}, \widetilde{X}, \rho, \rho_{\epsilon}
$$

as in the proof of Theorem 1.4, Step 1. By $\eta=\eta(\delta, w) \in(0,1)$ we denote a suitable constant to be chosen later.

Let $v:=w \circ G \circ \Phi \in L^{1}(I)$ and $I^{\prime} \Subset I$ be an open set such that spt $v \subset I^{\prime}$. If $v \equiv 0$ (i.e., $w \equiv 0$ ) it is enough to choose $u:=0$. If $v \neq \equiv 0$ we fix a sequence $\left(v_{k}\right)_{k} \subset C_{c}^{\infty}(I)$ such that

$$
v_{0} \equiv 0, \quad v_{k} \rightarrow v \text { in } L^{1}(I) \quad \text { and } \quad \operatorname{spt} v_{k} \subset I^{\prime} \forall k
$$

We require the following technical assumptions: $\left(v_{k}\right)_{k}$ is "rapidly converging", precisely

$$
\sum_{k=1}^{\infty}\left\|v_{k+1}-v_{k}\right\|_{L^{1}(I)} \leqslant \eta
$$

$v_{1}$ approximates "very well" $v$ in $L^{1}(I)$, so that

$$
\int_{I}\left|v_{1}-v\right||\rho| d \mathcal{L}^{n-1} \leqslant \eta
$$

and

$$
\left\|v_{k}\right\|_{L^{1}(I)} \leqslant 2\|v\|_{L^{1}(I)} \text { for any } k .
$$

We also fix, in a way we will specify along the proof, a decreasing infinitesimal sequence $\left(t_{k}\right)_{k}$. For any $k \in \mathbb{N}$ we consider a smooth function $h_{k}:\left[t_{k+1}, t_{k}\right] \rightarrow$ $[0,1]$ such that

$$
0 \leqslant h_{k}^{\prime} \leqslant \frac{1+\eta}{t_{k}-t_{k+1}}
$$

and

$$
h_{k} \equiv 0 \text { in a neighbourhood of } t_{k+1}, \quad h_{k} \equiv 1 \text { in a neighbourhood of } t_{k} .
$$

Define $\tilde{u} \in C^{\infty}(\widetilde{\Omega})$ by

$$
\tilde{u}(p):= \begin{cases}0 & \text { if } \tilde{f}(p)>t_{0} \\ h_{k}(\tilde{f}(p)) v_{k}\left(\pi_{I}(p)\right)+\left(1-h_{k}(\tilde{f}(p))\right) v_{k+1}\left(\pi_{I}(p)\right) & \text { if } t_{k+1}<\tilde{f}(p) \leqslant t_{k}\end{cases}
$$

where $\pi_{I}: I \times(-a, a) \rightarrow I$ is the canonical projection. We can choose $t_{0}$ so that

$$
\sup _{y \in I^{\prime}, t \in\left(0, t_{0}\right]}\left|\phi_{t}(y)\right|=\max \left\{\sup _{I^{\prime}}|\phi|, \sup _{I^{\prime}}\left|\phi_{t_{0}}\right|\right\}<a / 2 ;
$$


this is possible because

$$
\phi<\phi_{t}<\phi_{t_{0}} \forall t \in\left(0, t_{0}\right], \quad \sup _{I^{\prime}}|\phi|<a / 2, \quad \phi_{t} \rightarrow \phi \text { uniformly on } I^{\prime} .
$$

This choice implies spt $\tilde{u} \subset I^{\prime} \times(-a / 2, a / 2)$.

We point out that $\tilde{u}=v_{k} \circ \pi_{I}$ on $\partial \widetilde{\Omega}_{t_{k}}=\left\{\tilde{f}=t_{k}\right\}$ : roughly speaking, $\tilde{u}$ is defined on $\left\{t_{k+1}<\tilde{f}<t_{k}\right\}$ by interpolation between $v_{k+1} \circ \pi_{I}$ (on $\left.\widetilde{\partial} \Omega_{t_{k+1}}=\left\{\tilde{f}=t_{k+1}\right\}\right)$ and $v_{k} \circ \pi_{I}$ (on $\tilde{\partial} \Omega_{t_{k}}$ ).

By definition, $\tilde{u}$ is smooth on the open set $\left\{t_{k+1}<\tilde{f}<t_{k}\right\}$ for any $k$ and, by (5.28), it coincides with $v_{k} \circ \pi_{I}$ (that is smooth) on a neighbourhood of $\partial \widetilde{\Omega}_{t_{k}}=\left\{\tilde{f}=t_{k}\right\}$. This implies that $\tilde{u} \in C^{\infty}(\widetilde{\Omega})$ with $\tilde{u}=0$ out of $I^{\prime} \times(-a / 2, a / 2)$ and we can define $u \in C^{\infty}(\Omega)$ by

$$
u:=\tilde{u} \circ G^{-1} \text { on } \Omega \cap \mathcal{Q}, \quad u:=0 \text { on } \Omega \backslash \mathcal{Q} .
$$

We are going to prove that (1.3) holds for such $u$. To begin with, we notice that $T u=w$ because

$$
\tilde{u} \circ \Phi_{t} \rightarrow v \text { in } L^{1}(I)
$$

and, since the trace of a smooth function $u$ with support in $\mathcal{Q}$ is defined by the $L^{1}$ limit of $\tilde{u} \circ \Phi_{t}$ (see Step 1 in the proof of Theorem 1.4), we get precisely $T u=w$.

Step 2. Let us prove that $\int_{\Omega}|u| \leqslant \delta$. By the coarea formula, the inclusion $\Omega \cap$ spt $u \subset\left\{0<f<t_{0}\right\} \cap \mathcal{Q}$ and the inequality $|X f| \geqslant l$

$$
\begin{aligned}
\int_{\Omega} u d \mathcal{L}^{n} & =\int_{\Omega \cap \mathcal{Q}} u d \mathcal{L}^{n} \leqslant C_{1} \int_{\Omega \cap \mathcal{Q}} u|X f| \mathcal{L}^{n}=C_{1} \int_{0}^{t_{0}} \int_{\partial \Omega_{t} \cap \mathcal{Q}} u d\left|\partial \Omega_{t}\right|_{X} d t \\
& =C_{1} \int_{0}^{t_{0}} \int_{I}\left(\tilde{u} \circ \Phi_{t}\right)\left|\rho_{t}\right| d \mathcal{L}^{n-1} d t \leqslant C_{2} \int_{0}^{t_{0}} \int_{I}\left|\tilde{u} \circ \Phi_{t}\right| d \mathcal{L}^{n-1} d t \\
& \leqslant C_{2} \sum_{k=0}^{\infty} \int_{t_{k+1}}^{t_{k}} \int_{I}\left(h_{k}(t)\left|v_{k}\right|+\left(1-h_{k}(t)\right)\left|v_{k+1}\right|\right) d \mathcal{L}^{n-1} d t \\
& \leqslant C_{2} \sum_{k=0}^{\infty} \int_{t_{k+1}}^{t_{k}} \int_{I}\left(\left|v_{k}\right|+\left|v_{k+1}\right|\right) d \mathcal{L}^{n-1} d t \\
& \leqslant 4 C_{2} t_{0}\|v\|_{L^{1}(I)} d t,
\end{aligned}
$$

where we also used Remark 4.8, the fact that $\tilde{f} \circ \Phi_{t}=t$ and (5.27). The right hand side is smaller than $\delta$ provided $t_{0}$ is small enough.

Step 3. In order to derive the last inequality in (1.3), let us compute the horizontal derivatives of $\tilde{u}$. If $p$ is such that $t_{k+1}<\tilde{f}(p)<t_{k}$ we have

$$
\tilde{X}_{j} \tilde{u}(p)=\partial_{s_{n}} \tilde{u}(p)=h_{k}^{\prime}(\tilde{f}(p))\left[v_{k}\left(\pi_{I}(p)\right)-v_{k+1}\left(\pi_{I}(p)\right)\right] \tilde{X}_{j} \tilde{f}(p)
$$


while for $\ell \neq j$

$$
\begin{aligned}
\tilde{X}_{\ell} \tilde{u}(p)= & h_{k}^{\prime}(\tilde{f}(p))\left[v_{k}\left(\pi_{I}(p)\right)-v_{k+1}\left(\pi_{I}(p)\right)\right] \tilde{X}_{\ell} \tilde{f}(p) \\
& +h_{k}(\tilde{f}(p))\left\langle\nabla v_{k}\left(\pi_{I}(p)\right), d \pi_{I}\left[\tilde{X}_{\ell}(p)\right]\right\rangle \\
& +\left(1-h_{k}(\tilde{f}(p))\right)\left\langle\nabla v_{k+1}\left(\pi_{I}(p)\right), d \pi_{I}\left[\tilde{X}_{\ell}(p)\right]\right\rangle .
\end{aligned}
$$

By the smoothness of $\tilde{u}$, equalities (5.29) and (5.30) hold also if $\tilde{f}(p)=t_{k}$ or $\tilde{f}(p)=t_{k+1}$. In particular

$$
\tilde{X} \tilde{u}=\left(h_{k}^{\prime} \circ \tilde{f}\right)\left(v_{k} \circ \pi_{I}-v_{k+1} \circ \pi_{I}\right) \tilde{X} \tilde{f}+R \quad \text { on } \tilde{\Omega},
$$

where $R_{j} \equiv 0$ and

$$
\begin{aligned}
R_{\ell}(p)= & h_{k}(\tilde{f}(p))\left\langle\nabla v_{k}\left(\pi_{I}(p)\right), d \pi_{I}\left[\tilde{X}_{\ell}(p)\right]\right\rangle \\
& +\left(1-h_{k}(\tilde{f}(p))\right)\left\langle\nabla v_{k+1}\left(\pi_{I}(p)\right), d \pi_{I}\left[\tilde{X}_{\ell}(p)\right]\right\rangle
\end{aligned}
$$

for $\ell \neq j$. The coarea formula gives

$$
\begin{aligned}
\int_{\Omega}|X u| d \mathcal{L}^{n} & =\int_{\Omega \cap \mathcal{Q}}|X u| d \mathcal{L}^{n}=\int_{0}^{t_{0}} \int_{\partial \Omega_{t} \cap \mathcal{Q}} \frac{|X u|}{|X f|} d\left|\partial \Omega_{t}\right|_{X} d t \\
& =\sum_{k=0}^{\infty} \int_{t_{k+1}}^{t_{k}} \int_{I} \frac{\left|(\tilde{X} \tilde{u}) \circ \Phi_{t}\right|}{\left|(\tilde{X} \tilde{f}) \circ \Phi_{t}\right|}\left|\rho_{t}\right| d \mathcal{L}^{n-1} d t
\end{aligned}
$$

and, by (5.31) and the equality $\tilde{f} \circ \Phi_{t}=t$,

$$
\begin{aligned}
\int_{\Omega}|X u| d \mathcal{L}^{n} & \leqslant \sum_{k=0}^{\infty} \int_{t_{k+1}}^{t_{k}} \int_{I}\left[h_{k}^{\prime}(t)\left|v_{k}-v_{k+1}\right|+\frac{\left|R \circ \Phi_{t}\right|}{\left|\widetilde{X} \tilde{f} \circ \Phi_{t}\right|}\right]\left|\rho_{t}\right| d \mathcal{L}^{n-1} d t \\
& \leqslant \sum_{k=0}^{\infty} \int_{t_{k+1}}^{t_{k}} \int_{I}\left[\frac{1+\eta}{t_{k}-t_{k+1}}\left|v_{k}-v_{k+1}\right|+\frac{\left|R \circ \Phi_{t}\right|}{\left|\widetilde{X} \tilde{f} \circ \Phi_{t}\right|}\right]\left|\rho_{t}\right| d \mathcal{L}^{n-1} d t .
\end{aligned}
$$

Our strategy is the following: we are going to prove that the second addend in the right hand side of (5.33) gives a small contribution, while the first one is comparable to

$$
\int_{\partial \Omega}|w| d|\partial \Omega|_{X}=\int_{I}|v||\rho| d \mathcal{L}^{n-1}=\int_{t_{1}}^{t_{0}} \int_{I} \frac{|v|}{t_{0}-t_{1}}|\rho| d \mathcal{L}^{n-1} d t .
$$

For $\ell \neq j$ set

$$
\kappa_{\ell}:=\sup _{p \in \widetilde{\Omega}}\left|d \pi_{I}(p)\left[\tilde{X}_{\ell}(p)\right]\right| \leqslant \sup _{p \in \widetilde{\Omega}}\left|\tilde{X}_{\ell}(p)\right|<\infty
$$


by (5.32) one can estimate

$$
\begin{aligned}
\left|R_{\ell} \circ \Phi_{t}\right| & \leqslant\left(h_{k} \circ \tilde{f} \circ \Phi_{t}\right) \kappa_{\ell}\left|\nabla v_{k}\right|+\left(1-h_{k+1} \circ \tilde{f} \circ \Phi_{t}\right) \kappa_{\ell}\left|\nabla v_{k+1}\right| \\
& \leqslant \kappa_{\ell}\left(\left|\nabla v_{k}\right|+\left|\nabla v_{k+1}\right|\right) .
\end{aligned}
$$

Since $|\tilde{X} \tilde{f}| \geqslant l$, by Remark 4.8

$$
\begin{aligned}
\int_{t_{k+1}}^{t_{k}} \int_{I} \frac{\left|R \circ \Phi_{t}\right|}{\left|(\widetilde{X} \tilde{f}) \circ \Phi_{t}\right|}\left|\rho_{t}\right| d \mathcal{L}^{n-1} d t & \leqslant C_{3} \int_{t_{k+1}}^{t_{k}} \int_{I}\left(\left|\nabla v_{k}\right|+\left|\nabla v_{k+1}\right|\right) d \mathcal{L}^{n-1} d t \\
& =C_{3}\left(t_{k}-t_{k+1}\right)\left(\left\|\nabla v_{k}\right\|_{L^{1}(I)}+\left\|\nabla v_{k+1}\right\|_{L^{1}(I)}\right) .
\end{aligned}
$$

In particular, if $t_{k}-t_{k+1}$ is small enough we may assume that

$$
\int_{t_{k+1}}^{t_{k}} \int_{I} \frac{\left|R \circ \Phi_{t}\right|}{\left|(\tilde{X} \tilde{f}) \circ \Phi_{t}\right|}\left|\rho_{t}\right| d \mathcal{L}^{n-1} d t \leqslant 2^{-k-1} \eta
$$

Recalling (5.33) we achieve

$$
\int_{\Omega}|X u| d \mathcal{L}^{n} \leqslant(1+\eta) \sum_{k=0}^{\infty} \int_{t_{k+1}}^{t_{k}} \int_{I} \frac{\left|v_{k}-v_{k+1}\right|}{t_{k}-t_{k+1}}\left|\rho_{t}\right| d \mathcal{L}^{n-1} d t+\eta .
$$

In order to compare the series on the right hand side with (5.34), we are going to show that the summands can be made small whenever $k \geqslant 1$, so that one has to handle only the one corresponding to $k=0$. By Remark 4.8 and (5.25)

$$
\begin{aligned}
\sum_{k=1}^{\infty} \int_{t_{k+1}}^{t_{k}} \int_{I} \frac{\left|v_{k}-v_{k+1}\right|}{t_{k}-t_{k+1}}\left|\rho_{t}\right| d \mathcal{L}^{n-1} d t & \leqslant C_{4} \sum_{k=1}^{\infty} \int_{t_{k+1}}^{t_{k}} \int_{I} \frac{\left|v_{k}-v_{k+1}\right|}{t_{k}-t_{k+1}} d \mathcal{L}^{n-1} d t \\
& =C_{4} \sum_{k=1}^{\infty} \int_{I}\left|v_{k}-v_{k+1}\right| d \mathcal{L}^{n-1} \leqslant C_{4} \eta
\end{aligned}
$$

and since $v_{0} \equiv 0$

$$
\begin{aligned}
\int_{\Omega}|X u| d \mathcal{L}^{n} & \leqslant(1+\eta) \int_{t_{1}}^{t_{0}} \int_{I} \frac{\left|v_{0}-v_{1}\right|}{t_{0}-t_{1}}\left|\rho_{t}\right| d \mathcal{L}^{n-1} d t+(1+\eta) C_{4} \eta+\eta \\
& \leqslant(1+\eta) \int_{t_{1}}^{t_{0}} \int_{I} \frac{\left|v_{1}\right|}{t_{0}-t_{1}}\left|\rho_{t}\right| d \mathcal{L}^{n-1} d t+C_{5} \eta .
\end{aligned}
$$


By Remark 4.8, there exists $C_{6}>0$ such that $\left|\rho_{t}\right| \leqslant C_{6}|\rho|$ for any $t$. Using (5.26), (5.34) and the fact that $\eta<1$ we obtain

$$
\begin{aligned}
\int_{\Omega}|X u| d \mathcal{L}^{n} & \leqslant C_{7} \int_{t_{1}}^{t_{0}} \int_{I} \frac{\left|v_{1}\right|}{t_{0}-t_{1}}|\rho| d \mathcal{L}^{n-1} d t+C_{5} \eta \\
& =C_{7} \int_{I}\left|v_{1}\right||\rho| d \mathcal{L}^{n-1}+C_{5} \eta \\
& \leqslant C_{7}\left[\int_{I}|v||\rho| d \mathcal{L}^{n-1}+\eta\right]+C_{5} \eta \\
& =C_{7} \int_{\partial \Omega}|w| d|\partial \Omega|_{X}+C_{8} \eta
\end{aligned}
$$

and (1.3) follows for $\eta$ small enough. Notice that $C_{7}$ does not depend on $w$.

Step 4. We have to prove (1.4) in case $\partial \Omega$ is $X$-regular. By Remark 4.7, $\left|\rho_{t}\right| \rightarrow$ $|\rho|$ locally uniformly on $I$ and in particular (we are using again the fact that $\rho$ is bounded away from 0)

$$
\left|\rho_{t}\right| \leqslant(1+\eta)|\rho| \quad \text { on } I^{\prime} \quad \forall t \in\left(0, t_{0}\right)
$$

provided $t_{0}$ is small enough. From (5.35) and recalling that $v$ and $v_{1}$ are supported on $I^{\prime}$ one gets

$$
\begin{aligned}
\int_{\Omega}|X u| d \mathcal{L}^{n} & \leqslant(1+\eta)^{2} \int_{t_{1}}^{t_{0}} \int_{I} \frac{\left|v_{1}\right|}{t_{0}-t_{1}}|\rho| d \mathcal{L}^{n-1} d t+C_{5} \eta \\
& =(1+\eta)^{2} \int_{I}\left|v_{1}\right||\rho| d \mathcal{L}^{n-1} d t+C_{5} \eta \\
& \leqslant(1+\eta)^{2} \int_{I}|v||\rho| d \mathcal{L}^{n-1}+C_{9} \eta \\
& =(1+\eta)^{2} \int_{\partial \Omega}|w| d|\partial \Omega|_{X}+C_{9} \eta,
\end{aligned}
$$

where we have used again (5.26) and (5.34). Inequality (1.4) follows for small enough $\eta$.

Step 5. We now go back to Step 1 and consider the case $\Omega \cap \mathcal{Q}=\{f<0\}$. As in the proof of Theorem 1.4, Step 3, we introduce

$$
\hat{f}=-f, \quad \hat{X}=\left(X_{1}, \ldots,-X_{j}, \ldots, X_{m}\right) .
$$

Reasoning as before we can find $u \in C^{\infty}(\Omega) \cap W_{\hat{X}}^{1,1}(\Omega)$ (notice that $W_{\hat{X}}^{1,1}=W_{X}^{1,1}$ ) with support in $\mathcal{Q}$ and such that

$$
\hat{T} u=w, \quad \int_{\Omega}|u| d \mathcal{L}^{n} \leqslant \delta \quad \text { and } \quad \int_{\Omega}|\hat{X} u| d \mathcal{L}^{n} \leqslant C\|w\|_{L^{1}\left(\partial \Omega,|\partial \Omega|_{\hat{X}}\right)}
$$


and, in case $\partial \Omega$ is $X$-regular (or, which is the same, $\hat{X}$-regular),

$$
\int_{\Omega}|\hat{X} u| d \mathcal{L}^{n} \leqslant(1+\delta)\|w\|_{L^{1}\left(\partial \Omega,|\partial \Omega|_{\hat{X}}\right)} .
$$

As in the proof of Theorem 1.4, Step 3, we have denoted by $\hat{T} u$ the trace of $u$ on $\partial \Omega$ with respect to the CC structure induced by $\hat{X}$. It is now easy to prove that $u$ satisfies (1.3) and (1.4) (because $\hat{T} u=T u$ ) and this concludes the proof.

The following result is now an easy consequence of Theorems 1.5 and 5.3. The last part of the statement, loosely speaking, corresponds to choosing the number $t_{0}$, appearing in the proof of Theorem 1.5, "very small".

Corollary 5.4. Let $\Omega \subset \mathbb{R}^{n}$ be an $X$-Lipschitz domain with compact boundary and $u \in B V_{X}(\Omega)$. Then there exists $\bar{u} \in B V_{X}\left(\mathbb{R}^{n}\right)$ such that $\bar{u}_{\mid \Omega}=u$ and

$$
T u=T^{+} \bar{u}=T^{-} \bar{u} \quad|\partial \Omega|_{X} \text {-almost everywhere on } \partial \Omega .
$$

In particular, $|X \bar{u}|(\partial \Omega)=0$.

Moreover, for any compact set $\Omega^{\prime} \subset \mathbb{R}^{n}$ with $\Omega \Subset \Omega^{\prime}$ it is possible to choose $\bar{u}$ with support in $\Omega^{\prime}$.

We point out another interesting consequence of Theorem 1.5.

Corollary 5.5. Let $\Omega \subset \mathbb{R}^{n}$ be an $X$-Lipschitz domain with compact boundary and $u \in B V_{X}(\Omega)$. Then there exists a sequence $\left(u_{k}\right)_{k} \subset C^{\infty}(\Omega) \cap C^{0}(\bar{\Omega}) \cap B V_{X}(\Omega)$ such that, as $k \rightarrow \infty$,

$$
u_{k} \rightarrow u \text { in } L^{1}(\Omega) \text { and } \int_{\Omega}\left|X u_{k}\right| d \mathcal{L}^{n} \rightarrow|X u|(\Omega) .
$$

Proof. By Corollary 5.4, there exists $\bar{u} \in B V_{X}\left(\mathbb{R}^{n}\right)$ such that $\bar{u}=u$ on $\Omega$ and $|X \bar{u}|(\partial \Omega)=0$. If we fix a sequence $\left(\bar{u}_{k}\right)_{k} \subset C^{\infty}\left(\mathbb{R}^{n}\right) \cap B V_{X}\left(\mathbb{R}^{n}\right)$ such that

$$
\bar{u}_{k} \rightarrow \bar{u} \text { in } L^{1}\left(\mathbb{R}^{n}\right) \quad \text { and } \quad \int_{\mathbb{R}^{n}}\left|X \bar{u}_{k}\right| d \mathcal{L}^{n} \rightarrow|X \bar{u}|\left(\mathbb{R}^{n}\right)
$$

then, by Lemma 2.4, (5.36) holds with $u_{k}:=\bar{u}_{k \mid \Omega}$.

\subsection{Continuity of the trace operator}

As already mentioned in the introduction, the trace operator $T: B V_{X}(\Omega) \rightarrow$ $L^{1}\left(\partial \Omega,|\partial \Omega|_{X}\right)$ is not continuous if $B V_{X}(\Omega)$ is endowed with the topology of weak* convergence, see $e . g$. [2, page 181]. We are going to prove that, as in the classical case (see [2, Theorem 3.88]), $T$ is continuous when $B V_{X}(\Omega)$ is endowed with the topology induced by strict convergence. More precisely, we prove the following result. 
Theorem 5.6. Let $\Omega \subset \mathbb{R}^{n}$ be an $X$-Lipschitz domain with compact boundary and $u, u_{k} \in B V_{X}(\Omega)$ such that

$$
\lim _{k \rightarrow \infty} \int_{\Omega}\left|u_{k}-u\right| d \mathcal{L}^{n}=0 \text { and } \lim _{k \rightarrow \infty}\left|X u_{k}\right|(\Omega)=|X u|(\Omega) .
$$

Then $T u_{k} \rightarrow T u$ in $L^{1}\left(\partial \Omega,|\partial \Omega|_{X}\right)$.

The proof of Theorem 5.6, which follows the approach of [2, Theorem 3.88], will require some preliminary result. Given $\Omega$ (which we fix for the rest of this section) as in the statement of Theorem 5.6, let $\mathcal{Q}_{1}, \ldots, \mathcal{Q}_{q}$ be the open sets considered in the proof of Theorem 1.4. Since $\partial \Omega \subset \cup_{i=1}^{q} \mathcal{Q}_{i}$, it will be enough to prove that for any $i=1, \ldots, q$

$$
\left(T u_{k}\right)_{\mid \mathcal{Q}_{i}} \rightarrow(T u)_{\mid \mathcal{Q}_{i}} \quad \text { in } L^{1}\left(\partial \Omega,|\partial \Omega|_{X}\right)
$$

We then fix $i \in\{1, \ldots, q\}$ and $\mathcal{Q}=\mathcal{Q}_{i}$ together with the associated $f, j$ considered in the proof of Theorem 1.4, Step 1. We assume to fix ideas that $\Omega \cap \mathcal{Q}=\{f>0\}$; the case $\Omega \cap \mathcal{Q}=\{f<0\}$ will be treated later. With this assumption we can fix also

$$
I, a, G, \phi, \phi_{\epsilon}, \Phi, \Phi_{\epsilon}, \tilde{f}, \Omega_{\epsilon}, \widetilde{\Omega}, \widetilde{\Omega}_{\epsilon}, \tilde{X}_{\ell}, \tilde{X}, \rho, \rho_{\epsilon}, R_{\epsilon}
$$

as in the proof of Theorem 1.4.

Lemma 5.7. Let $u, u_{k} \in B V_{X}(\Omega)$ be such that $u_{k} \rightarrow u \in L^{1}(\Omega)$. Set $\tilde{u}:=u \circ G$ and $\tilde{u}_{k}:=u_{k} \circ G$ and assume that $\epsilon$ is small enough ${ }^{3}$ to define $v^{\epsilon}, v_{k}^{\epsilon}: I \rightarrow \mathbb{R}$ as

$$
v^{\epsilon}:=\frac{1}{\epsilon} \int_{0}^{\epsilon} \tilde{u} \circ \Phi_{t} d t, \quad v_{k}^{\epsilon}:=\frac{1}{\epsilon} \int_{0}^{\epsilon} \tilde{u}_{k} \circ \Phi_{t} d t .
$$

Then $v_{k}^{\epsilon} \rightarrow v^{\epsilon}$ in $L^{1}(I)$ as $k \rightarrow \infty$.

Proof. By Remark 4.8 and Theorem 2.6

$$
\begin{aligned}
\int_{I}\left|v_{k}^{\epsilon}-v^{\epsilon}\right| d \mathcal{L}^{n-1} & \leqslant \frac{1}{\epsilon} \int_{0}^{\epsilon} \int_{I}\left|\tilde{u}_{k} \circ \Phi_{t}-\tilde{u} \circ \Phi_{t}\right| d \mathcal{L}^{n-1} d t \\
& \leqslant \frac{C}{\epsilon} \int_{0}^{\epsilon} \int_{I}\left|\tilde{u}_{k} \circ \Phi_{t}-\tilde{u} \circ \Phi_{t}\right|\left|\rho_{t}\right| d \mathcal{L}^{n-1} d t \\
& =\frac{C}{\epsilon} \int_{0}^{\epsilon} \int_{\partial \Omega_{t} \cap \mathcal{Q}}\left|u_{k}-u\right| d\left|\partial \Omega_{t}\right|_{X} d t \\
& \leqslant \frac{C}{\epsilon} \int_{\Omega \cap \mathcal{Q}}\left|u_{k}-u\right||X f| d \mathcal{L}^{n} .
\end{aligned}
$$

The claim follows by the convergence of $u_{k}$ to $u$ in $L^{1}(\Omega)$ and the boundedness of $|X f|$.

${ }^{3}$ Precisely, we require that $\phi_{t}$ is defined on the whole $I$ for any $t \in(0, \epsilon)$. 
Lemma 5.8. Let $u \in B V_{X}(\Omega)$ and set $\tilde{u}:=u \circ G, v:=(T u) \circ G \circ \Phi: I \rightarrow \mathbb{R}$. Assume that $\epsilon$ is small enough to define $v^{\epsilon}:=\frac{1}{\epsilon} \int_{0}^{\epsilon} \tilde{u} \circ \Phi_{t} d t$. Then there exists $C=C(\mathcal{Q})>0$ such that

$$
\int_{I}\left|v-v^{\epsilon}\right| \leqslant C|X u|\left(\overline{G\left(R_{\epsilon}\right)}\right)
$$

Proof. If $u \in C^{\infty}(\Omega) \cap C^{0}(\bar{\Omega}) \cap B V_{X}(\Omega)$, then $T u=u_{\mid \partial \Omega}$ and

$$
\begin{aligned}
\int_{I}\left|v-v^{\epsilon}\right| d \mathcal{L}^{n-1} & \leqslant \frac{1}{\epsilon} \int_{0}^{\epsilon} \int_{I}\left|\tilde{u} \circ \Phi-\tilde{u} \circ \Phi_{t}\right| d \mathcal{L}^{n-1} d t \\
& \leqslant \frac{1}{\epsilon} \int_{0}^{\epsilon} \int_{I} \int_{\phi(y)}^{\phi_{t}(y)}\left|\partial_{s_{n}} \tilde{u}\left(y, \phi_{\tau}(y)\right)\right| d \tau d \mathcal{L}^{n-1}(y) d t \\
& \leqslant \int_{R_{\epsilon}}|\tilde{X} \tilde{u}| d \mathcal{L}^{n} \leqslant C_{1}|X u|\left(G\left(R_{\epsilon}\right)\right) .
\end{aligned}
$$

For a generic $u \in B V_{X}(\Omega)$, thanks to Corollary 5.5 we can fix a sequence $\left(u_{k}\right)_{k} \subset$ $C^{\infty}(\Omega) \cap C^{0}(\bar{\Omega}) \cap B V_{X}(\Omega)$ such that

$$
u_{k} \rightarrow u \text { in } L^{1}(\Omega) \text { and } \quad\left|X u_{k}\right|(\Omega) \rightarrow|X u|(\Omega) .
$$

Set $\tilde{u}_{k}:=u_{k} \circ G$ and

$$
v_{k}:=\left(T u_{k}\right) \circ G \circ \Phi, \quad v_{k}^{\epsilon}:=\frac{1}{\epsilon} \int_{0}^{\epsilon} \tilde{u}_{k} \circ \Phi_{t} d t .
$$

By Lemma 5.7 we have $v_{k}^{\epsilon} \rightarrow v^{\epsilon}$ in $L^{1}(I)$, thus for any $\ell=1, \ldots, m$

$$
v_{k}^{\epsilon} \rho_{\ell} \rightarrow v^{\epsilon} \rho_{\ell} \text { in } L^{1}(I) \text { as } k \rightarrow \infty
$$

because $\rho_{\ell} \in L^{\infty}(I)$. Moreover, for any $g \in C_{c}^{1}\left(\mathbb{R}^{n}, \mathbb{R}^{m}\right),|g| \leqslant 1$ we have by Lemma 2.5

$$
\lim _{k \rightarrow \infty} \int_{\Omega}\left\langle g, \sigma_{u_{k}}\right\rangle d\left|X u_{k}\right|=\int_{\Omega}\left\langle g, \sigma_{u}\right\rangle d|X u|
$$

and by (1.2)

$$
\begin{aligned}
\lim _{k \rightarrow \infty} \int_{\partial \Omega}\left\langle g, v_{\Omega}\right\rangle T u_{k} d|\partial \Omega|_{X} & =\lim _{k \rightarrow \infty}\left[\int_{\Omega} u_{k} \operatorname{div}_{X} g d \mathcal{L}^{n}+\int_{\Omega}\left\langle g, \sigma_{u_{k}}\right\rangle d\left|X u_{k}\right|\right] \\
& =\int_{\Omega} u \operatorname{div}_{X} g d \mathcal{L}^{n}+\int_{\Omega}\left\langle g, \sigma_{u}\right\rangle d|X u| \\
& =\int_{\partial \Omega}\left\langle g, v_{\Omega}\right\rangle T u d|\partial \Omega|_{X}
\end{aligned}
$$

This implies that for any $\ell=1, \ldots, m$

$$
\left(v_{\Omega}\right)_{\ell} T u_{k}|\partial \Omega|_{X} \rightarrow\left(v_{\Omega}\right)_{\ell} T u|\partial \Omega|_{X} \quad \text { as } k \rightarrow \infty
$$


in the sense of distributions, whence, as $k \rightarrow \infty$,

$$
v_{k} \rho_{\ell} \text { converge to } v \rho_{\ell} \text { in the sense of distributions on } I \text {. }
$$

Indeed, for any $\psi \in C_{c}^{\infty}(I)$ one can find $h_{\psi} \in C_{c}^{\infty}(I \times(-a, a))$ such that

$$
\psi=h_{\psi} \circ \Phi \text { on } I
$$

thus

$$
\begin{aligned}
\lim _{k \rightarrow \infty} \int_{I} \psi v_{k} \rho_{\ell} d \mathcal{L}^{n-1} & =\lim _{k \rightarrow \infty} \int_{I}\left(h_{\psi} \circ \Phi\right) v_{k} \rho_{\ell} d \mathcal{L}^{n-1} \\
& =\lim _{k \rightarrow \infty} \int_{\partial \Omega \cap \mathcal{Q}}\left(h_{\psi} \circ G^{-1}\right)\left(v_{\Omega}\right)_{\ell} T u_{k} d|\partial \Omega|_{X} \\
& =\int_{\partial \Omega \cap \mathcal{Q}}\left(h_{\psi} \circ G^{-1}\right)\left(v_{\Omega}\right)_{\ell} T u d|\partial \Omega|_{X} \\
& =\int_{I} \psi v \rho_{\ell} d \mathcal{L}^{n-1} .
\end{aligned}
$$

By (5.40) and (5.41) we get that, for any $\ell=1, \ldots, m,\left(v_{k}-v_{k}^{\epsilon}\right) \rho_{\ell}$ converges to $\left(v-v^{\epsilon}\right) \rho_{\ell}$ in the sense of distributions on $I$. This implies that

$$
\begin{aligned}
\int_{I}\left|v-v^{\epsilon}\right||\rho| d \mathcal{L}^{n-1} & =\sup _{\substack{\psi \in C_{c}^{\infty}\left(I, \mathbb{R}^{m}\right) \\
|\psi| \leqslant 1}} \int_{I}\left(v-v^{\epsilon}\right)\langle\rho, \psi\rangle d \mathcal{L}^{n-1} \\
& \leqslant \liminf _{k \rightarrow \infty} \int_{I}\left|v_{k}-v_{k}^{\epsilon}\right||\rho| d \mathcal{L}^{n-1}
\end{aligned}
$$

whence by (5.39) and Lemma 2.4

$$
\begin{aligned}
\int_{I}\left|v-v^{\epsilon}\right| & \leqslant C_{2} \int_{I}\left|v-v^{\epsilon}\right||\rho| d \mathcal{L}^{n-1} \leqslant C_{2} \liminf _{k \rightarrow \infty} \int_{I}\left|v_{k}-v_{k}^{\epsilon}\right||\rho| d \mathcal{L}^{n-1} \\
& \leqslant C_{3} \liminf _{k \rightarrow \infty} \int_{I}\left|v_{k}-v_{k}^{\epsilon}\right| d \mathcal{L}^{n-1} \leqslant C_{4} \liminf _{k \rightarrow \infty}\left|X u_{k}\right|\left(G\left(R_{\epsilon}\right)\right) \\
& \leqslant C_{4}|X u|\left(\overline{G\left(R_{\epsilon}\right)}\right) .
\end{aligned}
$$

We can now prove the continuity of the trace operator under strict convergence.

Proof of Theorem 5.6. As we said, it is enough to prove (5.37) for any fixed $i=$ $1, \ldots, q$. For $\epsilon>0$ small enough we define the real functions on $I$

$$
\begin{array}{ll}
v:=T u \circ G \circ \Phi & v_{k}:=T u_{k} \circ G \circ \Phi, \\
v^{\epsilon}:=\frac{1}{\epsilon} \int_{0}^{\epsilon} u \circ G \circ \Phi_{t} d t, & v_{k}^{\epsilon}:=\frac{1}{\epsilon} \int_{0}^{\epsilon} u_{k} \circ G \circ \Phi_{t} d t .
\end{array}
$$


We have

$$
\int_{I}\left|v-v_{k}\right| d \mathcal{L}^{n-1} \leqslant \int_{I}\left|v-v^{\epsilon}\right| d \mathcal{L}^{n-1}+\int_{I}\left|v^{\epsilon}-v_{k}^{\epsilon}\right| d \mathcal{L}^{n-1}+\int_{I}\left|v_{k}^{\epsilon}-v_{k}\right| d \mathcal{L}^{n-1}
$$

and by (5.38), Lemma 5.7 and Lemma 2.4

$$
\begin{aligned}
\limsup _{k \rightarrow \infty} \int_{\partial \Omega \cap \mathcal{Q}}\left|T u-T u_{k}\right| d|\partial \Omega|_{X} & \leqslant C_{1} \limsup _{k \rightarrow \infty} \int_{I}\left|v-v_{k}\right| d \mathcal{L}^{n-1} \\
& \leqslant C_{2}|X u|\left(\overline{G\left(R_{\epsilon}\right)}\right)+\underset{k \rightarrow \infty}{\limsup C_{2}\left|X u_{k}\right|\left(\overline{G\left(R_{\epsilon}\right)}\right)} \\
& \leqslant 2 C_{2}|X u|\left(\overline{G\left(R_{\epsilon}\right)}\right) .
\end{aligned}
$$

Notice that, if $\Omega \cap \mathcal{Q}=\{f<0\}$, one could reason as before to get

$$
\limsup _{k \rightarrow \infty} \int_{\partial \Omega \cap \mathcal{Q}}\left|T u-T u_{k}\right| d|\partial \Omega|_{\hat{X}} \leqslant 2 C_{2}|\hat{X} u|\left(\overline{G\left(R_{\epsilon}\right)}\right)=2 C_{2}|X u|\left(\overline{G\left(R_{\epsilon}\right)}\right)
$$

where $\hat{X}=\left(X_{1}, \ldots,-X_{j}, \ldots, X_{m}\right)$ (see also the proof of Theorem 1.4, Step 3). Thus (5.42) holds also in this second case and (5.37) follows as $\epsilon \rightarrow 0$.

\subsection{Traces vs. approximate limits in equiregular $\mathrm{CC}$ spaces}

This final part of the paper is devoted to the proof of Theorem 1.6.

Proof of Theorem 1.6. Let $\mathcal{Q}_{1}, \ldots, \mathcal{Q}_{q}$ be the open sets considered in the proof of Theorem 1.4. We can fix open subsets $\mathcal{Q}_{i}^{\prime} \Subset \mathcal{Q}_{i}$ such that

$$
\partial \Omega \subset \bigcup_{i=1}^{q} \mathcal{Q}_{i}^{\prime} .
$$

Fix $i \in\{1, \ldots, q\}$ and write $\mathcal{Q}=\mathcal{Q}_{i}, \mathcal{Q}^{\prime}=\mathcal{Q}_{i}^{\prime}$. By (5.43), it will be enough to prove that (1.5) holds for $|\partial \Omega|_{X}$-a.e. $z \in \partial \Omega \cap \mathcal{Q}^{\prime}$.

Let $f, j$ be as in the proof of Theorem 1.4, so that

$$
\partial \Omega \cap \mathcal{Q}=\{f=0\}, \quad f \in C^{\infty}(\mathcal{Q} \backslash \partial \Omega), \quad l:=\inf _{\mathcal{Q} \backslash \partial \Omega} X_{j} f>0 .
$$

As usual we assume that $\Omega \cap \mathcal{Q}=\{f>0\}$ and consider later the case $\Omega \cap \mathcal{Q}=$ $\{f<0\}$. With this assumption we can fix the corresponding

$$
I, a, G, \phi, \phi_{\epsilon}, \Phi, \Phi_{\epsilon}, \tilde{f}, \Omega_{\epsilon}, \widetilde{\Omega}, \widetilde{\Omega}_{\epsilon}, \tilde{X}_{\ell}, \tilde{X}, \rho, \rho_{\epsilon}
$$

considered in the proof of Theorem 1.4. Without loss of generality we may assume that $\mathcal{Q}^{\prime}=G\left(I^{\prime} \times(-b, b)\right)$ for suitable open set $I^{\prime} \Subset I$ and $b \in(a / 2, a)$. Let $L$ be the Lipschitz constant of $f, \pi_{I}$ the canonical projection $I \times(-a, a) \rightarrow I$ and consider $R>0$ such that $B\left(z,\left(1+\frac{L}{l}\right) R\right) \subset \mathcal{Q}$ for any $z \in \mathcal{Q}^{\prime}$. 
Let $z \in \partial \Omega \cap \mathcal{Q}^{\prime}$ be fixed. Since $|X f| \geqslant l$, for any $r<R$ one has

$$
\begin{aligned}
\int_{\Omega \cap B(z, r)}|u-T u(z)| d \mathcal{L}^{n} & \leqslant C_{1} \int_{\Omega \cap B(z, r)}|u-T u(z)||X f| d \mathcal{L}^{n} \\
& =C_{1} \int_{0}^{L r} \int_{\partial \Omega_{t} \cap B(z, r)}|u-T u(z)| d\left|\partial \Omega_{t}\right|_{X} d t
\end{aligned}
$$

where we have used the coarea formula and the fact that $f<\operatorname{Lr}$ on $B(z, r)$ which, in turn, is due to the Lipschitz continuity of $f$ and the equality $f(z)=0$. Let us define

$$
\begin{aligned}
& \tilde{z}:=G^{-1}(z)=\left(z^{\prime}, \phi\left(z^{\prime}\right)\right) \in \partial \widetilde{\Omega}, \text { where } z^{\prime}:=\pi_{I}\left(G^{-1}(z)\right), \\
& \widetilde{B}(\tilde{z}, r):=G^{-1}(B(z, r)), \\
& \tilde{u}:=u \circ G, \\
& v:=(T u) \circ G \circ \Phi \in L^{1}(I) .
\end{aligned}
$$

Then, by (5.44), Proposition 4.5 and Remark 4.8

$$
\begin{aligned}
\int_{\Omega \cap B(z, r)}|u-T u(z)| d \mathcal{L}^{n} & \leqslant C_{1} \int_{0}^{L r} \int_{\pi_{I}\left(\partial \widetilde{\Omega}_{t} \cap \widetilde{B}(\tilde{z}, r)\right)}\left|\tilde{u} \circ \Phi_{t}-v\left(z^{\prime}\right)\right|\left|\rho_{t}\right| d \mathcal{L}^{n-1} d t \\
& \leqslant C_{2} \int_{0}^{L r} \int_{\pi_{I}\left(\partial \tilde{\Omega}_{t} \cap \widetilde{B}(\tilde{z}, r)\right)}\left|\tilde{u} \circ \Phi_{t}-v\left(z^{\prime}\right)\right| d \mathcal{L}^{n-1} d t \\
\leqslant & C_{2} \int_{0}^{L r} \int_{\pi_{I}\left(\partial \widetilde{\Omega}_{t} \cap \widetilde{B}(\tilde{z}, r)\right)}\left|\tilde{u} \circ \Phi_{t}-v\right| d \mathcal{L}^{n-1} d t \\
& +C_{2} \int_{0}^{L r} \int_{\pi_{I}\left(\partial \tilde{\Omega}_{t} \cap \widetilde{B}(\tilde{z}, r)\right)}\left|v-v\left(z^{\prime}\right)\right| d \mathcal{L}^{n-1} d t .
\end{aligned}
$$

We are going to estimate separately the two integrals on the right hand side.

Step 1. Let us estimate the first integral in the right hand side of (5.45) assuming first that $u \in B V_{X}(\Omega) \cap C^{\infty}(\Omega) \cap C^{0}(\bar{\Omega})$, so that $v=\tilde{u} \circ \Phi$. For any $t \in(0, L r)$ we have

$$
\begin{aligned}
& \int_{\pi_{I}\left(\partial \widetilde{\Omega}_{t} \cap \widetilde{B}(\tilde{z}, r)\right)}\left|\tilde{u} \circ \Phi_{t}-v\right| d \mathcal{L}^{n-1} \\
\leqslant & \int_{\pi_{I}\left(\partial \widetilde{\Omega}_{t} \cap \tilde{B}(\tilde{z}, r)\right)} \int_{\phi(y)}^{\phi_{t}(y)}\left|\partial_{s_{n}} \tilde{u}(y, \sigma)\right| d \sigma d \mathcal{L}^{n-1}(y) \\
\leqslant & \int_{\{0<\tilde{f}<t\} \cap \pi_{I}^{-1}\left(\pi_{I}\left(\partial \tilde{\Omega}_{t} \cap \tilde{B}(\tilde{z}, r)\right)\right)}|\tilde{X} \tilde{u}| d \mathcal{L}^{n} .
\end{aligned}
$$


Notice that, if $s \in\{0<\tilde{f}<t\} \cap \pi_{I}^{-1}\left(\pi_{I}\left(\partial \widetilde{\Omega}_{t} \cap \widetilde{B}(\tilde{z}, r)\right)\right)$, then $s=\left(y, \phi_{\tau}(y)\right)=$ $\Phi_{\tau}(y)$ for some $y \in \pi_{I}\left(\partial \widetilde{\Omega}_{t} \cap \widetilde{B}(\tilde{z}, r)\right)$ and $\tau \in(0, t)$. We have by Lemma 2.2

$$
\begin{aligned}
L r & >t-\tau=\tilde{f}\left(\Phi_{t}(y)\right)-\tilde{f}\left(\Phi_{\tau}(y)\right) \\
& =\tilde{f}\left(\Phi_{t}(y)\right)-\tilde{f}\left(\exp \left(\left(\phi_{\tau}(y)-\phi_{t}(y)\right) \partial_{S_{n}}\right)\left(\Phi_{t}(y)\right)\right) \\
& \geqslant l\left(\phi_{t}(y)-\phi_{\tau}(y)\right)>0 .
\end{aligned}
$$

Since $G\left(\Phi_{\tau}(y)\right)=\exp \left(\left(\phi_{\tau}(y)-\phi_{t}(y)\right) X_{j}\right)\left(G\left(\Phi_{t}(y)\right)\right)$, by (5.47) and the definition of Carnot-Carathéodory distance

$$
d\left(G\left(\Phi_{t}(y)\right), G\left(\Phi_{\tau}(y)\right)\right) \leqslant\left|\phi_{t}(y)-\phi_{\tau}(y)\right| \leqslant \frac{L}{l} r .
$$

Since $y \in \pi_{I}\left(\partial \widetilde{\Omega}_{t} \cap \widetilde{B}(\tilde{z}, r)\right)$ we have

$$
G\left(\Phi_{t}(y)\right) \in B(z, r), \quad \text { i.e., } \quad d\left(z, G\left(\Phi_{t}(y)\right)\right)<r
$$

and in particular

$$
G\left(\Phi_{\tau}(y)\right) \in B\left(z,\left(1+\frac{L}{l}\right) r\right) .
$$

This implies that $s=\Phi_{\tau}(y) \in \widetilde{B}\left(\tilde{z},\left(1+\frac{L}{l}\right) r\right)$ and thus

$$
\{0<\tilde{f}<t\} \cap \pi_{I}^{-1}\left(\pi_{I}\left(\partial \widetilde{\Omega}_{t} \cap \widetilde{B}(\tilde{z}, r)\right)\right) \subset \widetilde{\Omega} \cap \widetilde{B}\left(\tilde{z},\left(1+\frac{L}{l}\right) r\right) .
$$

From (5.46) and a change of variable we obtain

$$
\begin{aligned}
\int_{\pi_{I}\left(\partial \widetilde{\Omega_{t}} \cap \tilde{B}(\tilde{z}, r)\right)}\left|\tilde{u} \circ \Phi_{t}-v\right| d \mathcal{L}^{n-1} & \leqslant \int_{\widetilde{\Omega} \cap \tilde{B}(\tilde{z},(1+L / l) r)}|\tilde{X} \tilde{u}| d \mathcal{L}^{n} \\
& \leqslant C_{3} \int_{\Omega \cap B(z,(1+L / l) r)}|X u| d \mathcal{L}^{n}
\end{aligned}
$$

whence

$$
\int_{0}^{L r} \int_{\pi_{I}\left(\partial \widetilde{\Omega}_{t} \cap \widetilde{B}(\tilde{z}, r)\right)}\left|\tilde{u} \circ \Phi_{t}-v\right| d \mathcal{L}^{n-1} d t \leqslant C_{4} r|X u|\left(\Omega \cap B\left(z,\left(1+\frac{L}{l}\right) r\right)\right) .
$$

Step 2. We have proved in Step 1 that, if $t \in(0, L r), y \in \pi_{I}\left(\partial \widetilde{\Omega}_{t} \cap \widetilde{B}(\tilde{z}, r)\right)$ and $\tau \in(0, t)$, then

$$
d\left(z, G\left(\Phi_{\tau}(y)\right)\right)<\left(1+\frac{L}{l}\right) r .
$$

One could check that the same argument works also for $\tau=0$ (it suffices to inspect the steps between (5.47) and (5.48) reading $\phi_{0}=\phi$ and $\left.\Phi_{0}=\Phi\right)$ and gives

$$
d(z, G(\Phi(y)))<\left(1+\frac{L}{l}\right) r,
$$


i.e., $(y, \phi(y)) \in \partial \widetilde{\Omega} \cap \widetilde{B}\left(\tilde{z},\left(1+\frac{L}{l}\right) r\right)$. This implies that

$$
\left.\pi_{I}\left(\partial \widetilde{\Omega}_{t} \cap \widetilde{B}(\tilde{z}, r)\right) \subset \pi_{I}\left(\partial \widetilde{\Omega} \cap \widetilde{B}\left(\tilde{z},\left(1+\frac{L}{l}\right) r\right)\right)\right) .
$$

Therefore we can estimate the second integral in the right hand side of (5.45) with

$$
\begin{aligned}
& \int_{0}^{L r} \int_{\pi_{I}\left(\partial \widetilde{\Omega} \widetilde{\Omega}_{t} \cap \widetilde{B}(\tilde{z}, r)\right)}\left|v-v\left(z^{\prime}\right)\right| d \mathcal{L}^{n-1} d t \\
\leqslant & L r \int_{\pi_{I}\left(\partial \widetilde{\Omega} \cap \widetilde{B}\left(\tilde{z},\left(1+\frac{L}{T}\right) r\right)\right)}\left|v-v\left(z^{\prime}\right)\right| d \mathcal{L}^{n-1} \\
\leqslant & C_{5} r \int_{\pi_{I}\left(\partial \widetilde{\Omega} \cap \widetilde{B}\left(\tilde{z},\left(1+\frac{L}{T}\right) r\right)\right)}\left|v-v\left(z^{\prime}\right)\right||\rho| d \mathcal{L}^{n-1} \\
= & C_{5} r \int_{\left.\partial \Omega \cap B\left(z,\left(1+\frac{L}{T}\right) r\right)\right)}|T u-T u(z)| d|\partial \Omega|_{X} .
\end{aligned}
$$

Step 3. It follows from (5.45), (5.49) and (5.50) that, if $u \in B V_{X}(\Omega) \cap C^{\infty}(\Omega) \cap$ $C^{0}(\bar{\Omega})$, then

$$
\begin{aligned}
& \int_{\Omega \cap B(z, r)}|u-T u(z)| d \mathcal{L}^{n} \\
\leqslant & C_{6} r\left[|X u|\left(\Omega \cap B\left(z,\left(1+\frac{L}{l}\right) r\right)\right)+\int_{\left.\partial \Omega \cap B\left(z,\left(1+\frac{L}{T}\right) r\right)\right)}|T u-T u(z)| d|\partial \Omega|_{X}\right]
\end{aligned}
$$

for any $r<R$. For a generic $u \in B V_{X}(\Omega)$ consider a sequence $\left(u_{k}\right)_{k} \subset B V_{X}(\Omega) \cap$ $C^{\infty}(\Omega) \cap C^{0}(\bar{\Omega})$ such that

$$
u_{k} \rightarrow u \text { in } L^{1}(\Omega) \quad \text { and } \quad\left|X u_{k}\right|(\Omega) \rightarrow|X u|(\Omega) .
$$

By Theorem 5.6 it follows that $T u_{k} \rightarrow T u$ in $L^{1}\left(\partial \Omega,|\partial \Omega|_{X}\right)$ and, in particular, $T u_{k} \rightarrow T u$ pointwise $|\partial \Omega|_{X}$-a.e. on $\partial \Omega$. If $z \in \partial \Omega \cap \mathcal{Q}^{\prime}$ is such that $T u_{k}(z) \rightarrow$ $T u(z)$, we obtain by (5.51) and Lemma 2.4

$$
\begin{aligned}
& \frac{1}{r^{Q}} \int_{\Omega \cap B(z, r)}|u-T u(z)| d \mathcal{L}^{n}=\frac{1}{r^{Q}} \lim _{k \rightarrow \infty} \int_{\Omega \cap B(z, r)}\left|u_{k}-T u_{k}(z)\right| d \mathcal{L}^{n} \\
& \leqslant \frac{C_{6}}{r^{Q-1}} \limsup _{k \rightarrow \infty}\left[\left|X u_{k}\right|\left(\Omega \cap B\left(z,\left(1+\frac{L}{l}\right) r\right)\right)\right. \\
& \left.+\int_{\left.\partial \Omega \cap B\left(z,\left(1+\frac{L}{T}\right) r\right)\right)}\left|T u_{k}-T u_{k}(z)\right| d|\partial \Omega|_{X}\right] \\
& \leqslant \frac{C_{6}}{r^{Q-1}}\left[|X u|\left(\Omega \cap \overline{B\left(z,\left(1+\frac{L}{l}\right) r\right)}\right)+\int_{\left.\partial \Omega \cap B\left(z,\left(1+\frac{L}{l}\right) r\right)\right)}|T u-T u(z)| d|\partial \Omega|_{X}\right] \\
& \leqslant \frac{C_{6}}{r^{Q-1}}\left[|X u|\left(\Omega \cap B\left(z, 2\left(1+\frac{L}{l}\right) r\right)\right)+\int_{\left.\partial \Omega \cap B\left(z,\left(1+\frac{L}{l}\right) r\right)\right)}|T u-T u(z)| d|\partial \Omega|_{X}\right] .
\end{aligned}
$$


It is a good point to notice that, in case $\Omega \cap \mathcal{Q}=\{f<0\}$, we would get for $|\partial \Omega|_{\hat{X}^{-a}}$.e. point $z \in \partial \Omega \cap \mathcal{Q}^{\prime}$

$$
\begin{aligned}
& \frac{1}{r^{Q}} \int_{\Omega \cap \hat{B}(z, r)}|u-T u(z)| d \mathcal{L}^{n} \\
\leqslant & \frac{C_{6}}{r^{Q-1}}\left[|\hat{X} u|\left(\Omega \cap \hat{B}\left(z, 2\left(1+\frac{L}{l}\right) r\right)\right)+\int_{\left.\partial \Omega \cap \hat{B}\left(z,\left(1+\frac{L}{T}\right) r\right)\right)}|T u-T u(z)| d|\partial \Omega|_{\hat{X}}\right]
\end{aligned}
$$

where as usual $\hat{X}=\left(X_{1}, \ldots,-X_{j}, \ldots, X_{m}\right)$ and we denote by $\hat{B}$ open balls with respect to the $\mathrm{CC}$ distance induced by $\hat{X}$. The previous inequality implies (5.52) because this distance obviously coincides with the one induced by $X$. See also the proof of Theorem 1.4, Step 3.

By Lemma 4.16 we obtain that for $|\partial \Omega|_{X}$-a.e. $z \in \partial \Omega \cap \mathcal{Q}^{\prime}$

$$
\lim _{r \rightarrow 0^{+}} \frac{|X u|\left(\Omega \cap B\left(z, 2\left(1+\frac{L}{l}\right) r\right)\right)}{r^{Q-1}}=0
$$

while from the Ahlfors regularity of $|\partial \Omega|_{X}$ and Lebesgue Theorem (see e.g. [27, Corollary 2.9.9]) one has for $|\partial \Omega|_{X}$-a.e. $z \in \partial \Omega \cap \mathcal{Q}^{\prime}$

$$
\begin{aligned}
& \lim _{r \rightarrow 0^{+}} \frac{1}{r Q-1} \int_{\left.\partial \Omega \cap B\left(z,\left(1+\frac{L}{T}\right) r\right)\right)}|T u-T u(z)| d|\partial \Omega|_{X} \\
\leqslant & \lim _{t \rightarrow 0^{+}} f_{\left.\partial \Omega \cap B\left(z,\left(1+\frac{L}{T}\right) r\right)\right)}|T u-T u(z)| d|\partial \Omega|_{X}=0 .
\end{aligned}
$$

Equality (1.5) follows from (5.52), (5.53) and (5.54), while (1.6) is a consequence of (1.5) and Lemma 4.15.

\section{References}

[1] L. Ambrosio, Some fine properties of sets of finite perimeter in Ahlfors regular metric measure spaces, Adv. Math. 159 (2001), 51-67.

[2] L. Ambrosio, N. Fusco and D. Pallara, "Functions of Bounded Variation and Free Discontinuity Problems", Oxford Mathematical Monographs. The Clarendon Press, Oxford University Press, New York, 2000..

[3] L. Ambrosio, F. Serra CASSAno and D. Vittone, Intrinsic regular hypersurfaces in Heisenberg groups, J. Geom. Anal. 16 (2006), 187-232.

[4] G. AnZellotti and M. Giaquinta, BV functions and traces (Italian), Rend. Sem. Mat. Univ. Padova 60 (1978), 1-21.

[5] G. ARENA and R. SERAPIONI, Intrinsic regular submanifolds in Heisenberg groups are differentiable graphs, Calc. Var. Partial Differential Equations 35 (2009), 517-536.

[6] H. BAhouri, J.-Y. Chemin and C.-J. XU, Trace theorem in Sobolev spaces associated with Hörmander's vector fields, In: "Partial Differential Equations and their Applications" (Wuhan, 1999), 1-14, World Sci. Publ., River Edge, NJ, 1999. 
[7] H. Bahouri, J.-Y. Chemin and C.-J. XU, Trace and trace lifting theorems in weighted Sobolev spaces, J. Inst. Math. Jussieu 4 (2005), 509-552.

[8] H. BAhouri, J.-Y. Chemin and C.-J. XU, Trace theorem on the Heisenberg group, Ann. Inst. Fourier (Grenoble) $\mathbf{5 9}$ (2009), 491-514.

[9] A. BALdi and F. MontefalCone, A note on the extension of BV functions in metric measure spaces, J. Math. Anal. Appl. 340 (2008), 197-208.

[10] A. BellaïCHE and J.-J. RISLER (editors), "Sub-Riemannian Geometry", Progress in Mathematics, Vol. 144, Birkhäuser Verlag, Basel, 1996.

[11] S. BERHANU and I. PESENSON, The trace problem for vector fields satisfying Hörmander's condition, Math. Z. 231 (1999), 103-122.

[12] F. BigOlin and F. SERRA CASSANO, Intrinsic regular graphs in Heisenberg groups vs. weak solutions of non-linear first-order PDEs, Adv. Calc. Var. 3 (2010), 69-97.

[13] F. BIgOlin and F. SERra CASSANO, Distributional solutions of Burgers' equation and intrinsic regular graphs in Heisenberg groups, J. Math. Anal. Appl. 366 (2010), 561-568.

[14] M. BIroli and U. Mosco, Sobolev and isoperimetric inequalities for Dirichlet forms on homogeneous spaces, Atti Accad. Naz. Lincei, Cl. Sci. Fis. Mat. Nat., IX. Ser. Rend. Lincei, Mat. Appl., 6 (1995), 37-44.

[15] A. Bonfiglioli, E. LANCONELli and F. UguZzoni, "Stratified Lie Groups and Potential Theory for their sub-Laplacians", Springer Monographs in Mathematics. Springer, Berlin, 2007.

[16] L. Capogna, D. Danielli and N. Garofalo, The geometric Sobolev embedding for vector fields and the isoperimetric inequality, Comm. Anal. Geom. 2 (1994), 203-215.

[17] L. CAPOGNA and N. GAROFALO, Ahlfors type estimates for perimeter measures in CarnotCarathéodory spaces, J. Geom. Anal. 16 (2006), 455-497.

[18] L. Capogna, N. Garofalo and D.-M. Nhieu, A version of a theorem of Dahlberg for the subelliptic Dirichlet problem, Math. Res. Lett. 5 (1998), 541-549.

[19] L. Capogna, N. Garofalo and D.-M. Nhieu, Properties of harmonic measures in the Dirichlet problem for nilpotent Lie groups of Heisenberg type, Amer. J. Math. 124 (2002), 273-306.

[20] L. Capogna, N. Garofalo \& D.-M. Nhieu, Mutual absolute continuity of harmonic and surface measures for Hörmander type operators, In: "Perspectives in Partial Differential Equations, Harmonic Analysis and Applications", 49-100, Proc. Sympos. Pure Math., 79, Amer. Math. Soc., Providence, RI, 2008.

[21] G. CitTI and M. MANFREDINI, Blow-up in non homogeneous Lie groups and rectifiability, Houston J. Math. 31 (2005), 333-353.

[22] G. CITTI and M. MANFREDINI, Implicit function theorem in Carnot-Carathéodory spaces, Commun. Contemp. Math. 8 (2006), 657-680.

[23] D. DANIELLI, A Fefferman-Phong type inequality and applications to quasilinear subelliptic equations, Potential Anal. 11 (1999), 387-413.

[24] D. Danielli, N. Garofalo and D.-M. Nhieu, Trace inequalities for CarnotCarathéodory spaces and applications, Ann. Scuola Norm. Sup. Pisa Cl. Sci. 27 (1998), 195-252.

[25] D. Danielli, N. Garofalo and D.-M. Nhieu, Sub-elliptic Besov spaces and the characterization of traces on lower dimensional manifolds, In: "Harmonic Analysis and Boundary Value Problems" (Fayetteville, AR, 2000), 19-37, Contemp. Math. 277, Amer. Math. Soc., Providence, RI, 2001.

[26] D. Danielli, N. Garofalo and D.-M. Nhieu, "Non-doubling Ahlfors Measures, Perimeter Measures, and the Characterization of the Trace Spaces of Sobolev Functions in Carnot-Carathéodory Spaces", Mem. Amer. Math. Soc. 182 (2006).

[27] H. FEDERER, "Geometric Measure Theory", Die Grundlehren der mathematischen Wissenschaften, Band 153 Springer-Verlag New York Inc., New York, 1969.

[28] G. B. Folland, Subelliptic estimates and function spaces on nilpotent Lie groups, Ark. Mat. 13 (1975), 161-207. 
[29] G. B. Folland and E. M. Stein, "Hardy Spaces on Homogeneous Groups", Princeton University Press, 1982.

[30] B. Franchi, S. Gallot and R. L. WheEden, Sobolev and isoperimetric inequalities for degenerate metrics, Math. Ann. 300 (1994), 557-571.

[31] B. Franchi, R. SERAPIONI and F. Serra CASSANo, Meyers-Serrin type theorems and relaxation of variational integrals depending vector fields, Houston J. Math. 22 (1996), 859-889.

[32] B. Franchi, R. Serapioni and F. Serra Cassano, Approximation and imbedding theorems for weighted Sobolev spaces associated with Lipschitz continuous vector fields, Boll. Un. Mat. Ital. B (7) 11 (1997), 83-117.

[33] B. Franchi, R. SERAPIONI and F. SERra CASSANo, Rectifiability and perimeter in the Heisenberg group, Math. Ann. 321 (2001), 479-531.

[34] B. FrANCHI, R. SERAPIONI and F. SERRA CASSANO, On the structure of finite perimeter sets in step 2 Carnot groups, J. Geom. Anal. 13 (2003), 421-466.

[35] B. Franchi, R. SERAPIONI and F. SERRA CASSANO, Regular hypersurfaces, intrinsic perimeter and implicit function theorem in Carnot groups, Comm. Anal. Geom. 11 (2003), 909-944.

[36] B. Franchi, R. Serapioni and F. Serra CASSAno, Intrinsic Lipschitz graphs in Heisenberg groups, J. Nonlinear Convex Anal. 7 (2006), 423-441.

[37] B. Franchi, R. SERAPIONI and F. SERRA CASSANO, Regular submanifolds, graphs and area formula in Heisenberg groups, Adv. Math. 211 (2007), 152-203.

[38] B. Franchi, R. Serapioni and F. Serra Cassano, Differentiability of intrinsic Lipschitz functions within Heisenberg groups, J. Geom. Anal. 21 (2011), 1044-1084.

[39] N. Garofalo and D.-M. NhIEU, Isoperimetric and Sobolev inequalities for CarnotCarathéodory spaces and the existence of minimal surfaces, Commun. Pure Appl. Math. 49 (1996), 1081-1144.

[40] N. GAROFALO and D.-M. NHIEU, Lipschitz continuity, global smooth approximations and extension theorems for Sobolev functions in Carnot-Carathéodory spaces, J. Anal. Math. 74 (1998), 67-97.

[41] E. Giusti, "Minimal Surfaces and Functions of Bounded Variation", Monographs in Mathematics, 80. Birkhäser Verlag, Basel, 1984.

[42] B. KirchHeim and F. SerRa Cassano, Rectifiability and parametrization of intrinsic regular surfaces in the Heisenberg group, Ann. Sc. Norm. Super. Pisa Cl. Sci. 3 (2004), 871-896.

[43] V. MagnAni, "Elements of Geometric Measure Theory on Sub-Riemannian Groups", PhD thesis, Scuola Normale Superiore, Pisa, 2002.

[44] V. Magnani, Characteristic points, rectifiability and perimeter measure on stratified groups, J. Eur. Math. Soc. (JEMS) 8 (2006), 585-609.

[45] V. MAGNANi and D. VitTONE, An intrinsic measure for submanifolds in stratified groups, J. Reine Angew. Math. 619 (2008), 203-232.

[46] N. G. MEYERS and W. P. ZIEMER, Integral inequalities of Poincaré and Wirtinger type for BV functions, Amer. J. Math. 99 (1977), 1345-1360.

[47] M. Miranda, Comportamento delle successioni convergenti di frontiere minimali (Italian), Rend. Sem. Mat. Univ. Padova 38 (1967), 238-257.

[48] J. Mitchell, On Carnot-Carathéodory metrics, J. Differential Geom. 21 (1985), 35-45.

[49] R. MonTI and D. MoRBIDELLI, Trace theorems for vector fields, Math. Z. 239 (2002), 747-776.

[50] R. Monti and F. Serra Cassano, Surface measures in Carnot-Carathéodory spaces, Calc. Var. Partial Differential Equations 13 (2001), no. 3, 339-376.

[51] D. Morbidelli, Fractional Sobolev norms and structure of Carnot-Carathéodory balls for Hörmander vector fields, Studia Math. 139 (2000), 213-244.

[52] A. NAGel, E. M. SteIn and S. WAinger, Balls and metrics defined by vector fields I: basic properties, Acta Math. 155 (1985), 103-147. 
[53] L. RothSCHILD and E. M. STEIN, Hypoelliptic differential operatorsand nilpotent groups, Acta Math. 137 (1976), 247-320.

[54] C. SELBY, An extension and trace theorem for functions of H-bounded variation in Carnot groups of step 2, Houston J. Math. 33 (2007), 593-616 (electronic).

[55] L. Simon, Lectures on geometric measure theory, In: "Proceedings of the Centre for Mathematical Analysis, Australian National University", Vol. 3, Centre for Mathematical Analysis, Canberra, 1983.

[56] E. STEIN, "Harmonic Analysis: Real-variable Methods, Orthogonality, and Oscillatory Integrals", Princeton Mathematical Series, 43. Monographs in Harmonic Analysis, III. Princeton University Press, Princeton, NJ, 1993.

[57] N. Th. VAropoulos, L. SalofF-Coste and T. Coulhon, "Analysis and Geometry on Groups", Cambridge Tracts in Mathematics, Vol. 100, Cambridge University Press, Cambridge, 1992.

[58] F. Vigneron, The trace problem for Sobolev spaces over the Heisenberg group, J. Anal. Math. 103 (2007), 279-306.

[59] D. VitTone, "Submanifolds in Carnot Groups", Tesi di Perfezionamento, Scuola Normale Superiore, Pisa, 2008.

Dipartimento di Matematica

Università di Padova

Via Trieste, 63

35121 Padova, Italia

vittone@math.unipd.it 